\title{
Inference of hot star density stream properties from data on rotationally recurrent DACs ${ }^{\star}$
}

\author{
J. C. Brown ${ }^{1,2}$, R. K. Barrett ${ }^{1}$, L. M. Oskinova ${ }^{1,3}$, S. P. Owocki ${ }^{1,4}$, W.-R. Hamann ${ }^{3}$, J. A. de Jong ${ }^{2,5}$, \\ L. Kaper ${ }^{2}$, and H. F. Henrichs ${ }^{2}$ \\ 1 Department of Physics and Astronomy, University of Glasgow, Glasgow, G12 8QQ, Scotland, UK \\ 2 Astronomical Institute "Anton Pannekoek", University of Amsterdam, Kruislaan 403, 1098 SJ Amsterdam, The Netherlands \\ 3 Professur Astrophysik, Universitat Potsdam, Am Neuen Palais 10, 14469 Potsdam, Germany \\ ${ }^{4}$ Bartol Research Institute, University of Delaware, Newark, DE 19716, USA \\ ${ }^{5}$ Leiden Observatory, University of Leiden, Niels Bohrweg 2, 2333 CA Leiden, The Netherlands
}

Received 16 June 2003 / Accepted 15 September 2003

\begin{abstract}
The information content of data on rotationally periodic recurrent discrete absorption components (DACs) in hot star wind emission lines is discussed. The data comprise optical depths $\tau(w, \phi)$ as a function of dimensionless Doppler velocity $w=\left(\Delta \lambda / \lambda_{0}\right)\left(c / v_{\infty}\right)$ and of time expressed in terms of stellar rotation angle $\phi$. This is used to study the spatial distributions of density, radial and rotational velocities, and ionisation structures of the corotating wind streams to which recurrent DACs are conventionally attributed.

The simplifying assumptions made to reduce the degrees of freedom in such structure distribution functions to match those in the DAC data are discussed and the problem then posed in terms of a bivariate relationship between $\tau(w, \phi)$ and the radial velocity $v_{\mathrm{r}}(r)$, transverse rotation rate $\Omega(r)$ and density $\rho(r, \phi)$ structures of the streams. The discussion applies to cases where: the streams are equatorial; the system is seen edge on; the ionisation structure is approximated as uniform; the radial and transverse velocities are taken to be functions only of radial distance but the stream density is allowed to vary with azimuth. The last kinematic assumption essentially ignores the dynamical feedback of density on velocity and the relationship of this to fully dynamical models is discussed. The case of narrow streams is first considered, noting the result of Hamann et al. (2001) that the apparent acceleration of a narrow stream DAC is higher than the acceleration of the matter itself, so that the apparent slow acceleration of DACs cannot be attributed to the slowness of stellar rotation. Thus DACs either involve matter which accelerates slower than the general wind flow, or they are formed by structures which are not advected with the matter flow but propagate upstream (such as Abbott waves). It is then shown how, in the kinematic model approximation, the radial speed of the absorbing matter can be found by inversion of the apparent acceleration of the narrow DAC, for a given rotation law.

The case of broad streams is more complex but also more informative. The observed $\tau(w, \phi)$ is governed not only by $v_{\mathrm{r}}(r)$ and $\Omega(r)$ of the absorbing stream matter but also by the density profile across the stream, determined by the azimuthal $\left(\phi_{0}\right)$ distribution function $F_{0}\left(\phi_{0}\right)$ of mass loss rate around the stellar equator. When $F_{0}\left(\phi_{0}\right)$ is fairly wide in $\phi_{0}$, the acceleration of the DAC peak $\tau(w, \phi)$ in $w$ is generally slow compared with that of a narrow stream DAC and the information on $v_{\mathrm{r}}(r), \Omega(r)$ and $F_{0}\left(\phi_{0}\right)$ is convoluted in the data $\tau(w, \phi)$.

We show that it is possible, in this kinematic model, to recover by inversion, complete information on all three distribution functions $v_{\mathrm{r}}(r), \Omega(r)$ and $F_{0}\left(\phi_{0}\right)$ from data on $\tau(w, \phi)$ of sufficiently high precision and resolution since $v_{\mathrm{r}}(r)$ and $\Omega(r)$ occur in combination rather than independently in the equations. This is demonstrated for simulated data, including noise effects, and is discussed in relation to real data and to fully hydrodynamic models.
\end{abstract}

Key words. stars: early-type - stars: winds, outflows - stars: mass-loss - line: profiles

\section{Introduction}

The phenomenon of Discrete Absorption Components (DACs) moving (often recurrently) in the broad emission line profiles of hot star winds has been discussed extensively in the

Send offprint requests to: J. C. Brown,

e-mail: john@astro.gla.ac.uk

$\star$ Figures 6, 7, 9, 10,12,13,15 and 16 are only available in electronic form at http://www. edpsciences.org literature - see e.g., Prinja \& Howarth (1988), Owocki et al. (1995), Henrichs et al. (1994), Fullerton et al. (1997) and recent overviews of data and theoretical interpretation by Kaper (2000) and by Cranmer \& Owocki (1996) respectively. In the present paper we summarise some major aspects of DAC interpretation in relation to the information content of the data, and examine how analytic aspects of a simplified kinematic model enable formulation of DAC modelling from a diagnostic inverse problem viewpoint (Craig \& Brown 1986). This may 
provide a useful tool in the quantitative non-parametric interpretation of recurrent DAC data sets from specific stars.

The present situation can be summarised as follows.

1. DACs are attributed to structures, accelerating outward in stellar winds, which have enhanced optical depth over a rather narrow range of Doppler wavelengths compared to the overall absorption line width.

2. This is attributed to enhanced density and/or reduced velocity gradient along the line of sight; that is, the increase in the number of absorbers per unit velocity can be attributed to an actual increase in spatial density, or to an increase in the spatial volume over which the absorbers have that velocity.

3. In the case of recurrent DACs the periodicity is commonly attributed to stellar rotation and the distribution pattern of absorbing material is taken to be time-independent in the stellar rotation frame, though the matter itself moves through this pattern (that is, the flow is stationary in the corotating frame, but not static). This is the type of DAC phenomenon we will consider here.

4. There are suggestions (e.g., Hamann et al. 2001) that some periodicity should be attributed to causes other than rotation (e.g., non-radial pulsation), and even debate over whether "DAC" is the correct terminology for some Component features, even though they are Discrete and in Absorption!

5. The existence of such corotating patterns of enhanced density/reduced velocity gradient is often attributed to corotating interaction regions (Mullan 1984). These CIRs arise where outflows with different radial speeds from azimuthully distinct regions collide.

6. Cranmer \& Owocki (1996) have modelled the creation of CIRs physically by studying the hydrodynamic response of a radiatively driven wind to empirical imposition of bright spots azimuthally localised on the stellar surface. Their simulations predict DAC profiles and time dependence generally similar to data and have provided the best insight yet into the interpretation of DACs, such as the relative importance in the absorbing matter patterns of deviations in density and in velocity gradients from the mean wind. It is central to these dynamical models that the absorbing pattern is created by variation in the outflow speed with azimuth as well as radius and that the inertia of the enhanced density reacts back on the velocity field.

7. On the other hand, progress has been made in the interpretation of specific DAC data sets by use of a purely kinematic approach (Owocki et al. 1995; Fullerton et al. 1997). In this the absorption is attributed to a rotating density pattern following radial (and rotational) velocity laws which are the same at all azimuths (though not in general the same velocity laws as the mean wind).

8. Neither the kinematic nor the dynamical approach is entirely satisfactory. The former ignores the dynamical feedback of density on velocity. The latter, on the other hand, necessarily involves non-monotonic velocity variation along the line of sight, creating ambiguity in identifying Doppler velocities with distances (cf. Brown et al. 1997 discussion of emission line profiles). Secondly, matching the DAC data set from a particular object requires the hydro code to be run for sets of radiative driver (e.g., hotspot) properties occupying a large range of parameter space.

In this paper we approach the DAC diagnostic problem from a different viewpoint, aiming to assist ultimate integration of the kinematic and dynamical approaches. While forward modelling of CIRs using radiation hydrodynamics (as in Cranmer \& Owocki 1996) allows detailed predictions of line-profile variations to be made, it does not permit the sensitivity of the data to the physical characteristics of the stellar wind to be assessed (that is, finding a model that fits the data does not, in itself, preclude the possibility that there are other very different models that fit equally well). Moreover, it is not possible to examine the accuracy of the physical assumptions that go into radiation hydrodynamical modelling. For these reasons we develop a model of rotationally-recurrent line profile variations that makes no specific dynamical assumptions but instead adopts a purely kinematical approach: given any (axisymmetric - see Sect. 2) wind velocity law we can calculate the line-profile variations that would result from any variation of mass-loss rate over the surface of the star, and we investigate the inverse problem (Craig \& Brown 1986) of inferring the stellar wind velocity law (and density variation) given an observed line-profile variation (i.e., given a dynamical spectrum). That is, we formulate the problem in approximate kinematic fashion ( 7 , above) but address it as an inverse problem. Though still subject to the objection of ignoring the detailed dynamical feedback of density on velocity, this may enable inference of approximate forms of the absorber density and velocity distribution with radius along the line of sight without restrictive parametric assumptions regarding these forms, such as a $\beta$-law velocity law, which can never reveal the presence of a plateau in the wind velocity. The intention is to create a means to find a "corotating dense region" (CDR) approximation to the actual CIR structure as the starting point of a search for a physically consistent structure using a full dynamical treatment along the lines of Cranmer \& Owocki (1996). The attempt by de Jong (2000) to carry out DAC diagnosis used a kinematic model with a Genetic Algorithm search of a parametric density model space with prescribed radial velocity law shows how time consuming such searches can be. It also shows, as discussed later, how a restrictive parametrisation may lead to failure to obtain a satisfactory fit to the data. While emphasising, and exploring for the first time, the inverse formulation we recognise that the forward fitting approach can be valuable in terms of testing for the presence and magnitude of specific preconceived model features.

We first (Sect. 2) discuss the information content of recurrent DAC data and the need to make assumptions concerning the wind structure in order to reduce the degrees of freedom in the model to match those in the data, whether by forward fitting or inverse inference. With these assumptions we then derive the basic equations relating the DAC properties in the kinematic approximation to the density and velocity distributions with radius for a general CDR which may be broad in azimuth (cf. discussions in Hamann et al. 2001 of the narrow stream case). 
In Sect. 3 we summarise the analytic properties of the narrow-stream CDR case, first in the forward modelling approach (cf. Hamann et al. 2001), then as an inverse problem of inferring the CDR matter velocity law non-parametrically from DAC acceleration data. We set out the narrow-stream inversion procedure given a wind rotation law and without the need to use mass continuity, and discuss the fact that our model assumption of an axisymmetric wind velocity law is not strictly necessary in this case. The relationship of narrow-stream to wide-stream inversions is considered. In Sect. 4 we tackle the problem of a general wide CDR showing how its density and velocity distibutions are reflected in the DAC profile and its recurrent time variation. Note that Hamann et al. (2001) only addressed the time dependence of the narrow DAC wavelength and not the DAC profile, and that in most treatments (e.g., Prinja \& Howarth 1988; Owocki et al. 1995) addressing the DAC profile, only a parametric fit (e.g., Gaussian) is used, rather than the full information present in the profile. We then address the inverse problem of inferring non-parametrically the $\mathrm{CDR} /$ stream velocity and density structure from full data on the time-varying wide DAC profile, and illustrate in Sect. 5, using synthetic data, the success of the method within the restrictions of the kinematic approach. Finally in Sect. 6 we discuss how future work may integrate this inverse CDR diagnostic formalism with full dynamical modelling to enhance our ability to model the true CIR structure of specific stars from their recurrent DAC data sets.

\section{Kinematic formulation of the DAC interpretation problem}

The blue wing of the P Cygni profile of a hot-star wind spectral line contains an absorption component (from moving material in the wind absorbing the stellar continuum) and a scattered component (from the wind volume). Since we are interested in DACs we want to remove the scattered light leaving only the absorption component. This requires a careful treatment (Massa et al. 1995, 2003). In addition, it will be seen in this section that for the kinematical model that we develop the optical depth profile depends linearly on the surface density (i.e., mass loss rate) variation, for a given wind velocity law. It follows from this that we could examine the absorption component of the whole wind, or we could consider only the optical depth excess related to the DAC overdensity itself. The latter may be simpler in practice to obtain, by subtraction of a "least absorption" wind absorption line profile, say (e.g., Kaper et al. 1999). In the results presented here we generally assume that we have the optical depth excess corresponding to the DAC, but this is not necessary for the formalism we develop.

In any event, we suppose here that high resolution spectral line data can be processed so as to extract the absorption component (or the absorption component of a single recurrent DAC feature) from the overall line profile. Then the recurrent DAC data can be expressed in terms of the DAC optical depth $\tau$ as a function of Doppler shift $\Delta \lambda$ and time $t$ related to the observer azimuth $\phi$, measured relative to a convenient reference point in the frame of the star, rotating at angular speed $\Omega_{0}$, by $t=\phi / \Omega_{0}$.
The data function $\tau(\Delta \lambda, \phi)$ of two variables for a single line is clearly incapable of diagnosing the full 3-D structure of even a steady state general wind which involves at least the mass density $\rho$, velocity $\boldsymbol{v}$ and temperature $T$ as functions of 3 -D vector position $\boldsymbol{r}$, and the inclination $i$. Clearly, the structure inference inversion problem to determine these four functions of three variables (along with the inclination) from the single function, $\tau(\Delta \lambda, \phi)$, of two variables is massively underdetermined. Correspondingly, in any forward modelling of the $\tau(\Delta \lambda, \phi)$ data from a theoretical structure, there may be a multiplicity of $\rho, \boldsymbol{v}, T$ distributions which fit the data. Progress in either approach can only be made then by introducing a number of simplifying assumptions about the geometry etc., and by utilising the physics of the situation.

In the work presented here we make one important physical approximation regarding the nature of the wind velocity law, and a number of assumptions (mostly geometrical) of lesser importance, which do not critically affect to the conclusions we reach but greatly simplify the presentation.

Model assumption: The wind velocity is assumed to be axisymmetric, that is, the radial flow speed $v_{\mathrm{s}}(r)$ of matter in the absorbing stream (and the rotation rate $\Omega(r)$ ) is independent of $\phi$. In addition, we assume that the velocity law is (approximately) monotonic. In general, this velocity law need not be the same as the flow speed law in the mean wind (see remark 8 in Sect. 1).

Note that the principal consequence of this assumption is that all streamlines in the corotating frame are obtained from a single streamline (see Eq. (3) by shifting in azimuth: the pattern of streamlines is also axisymmetric.

Although dynamical simulations do not satisfy $v_{\mathrm{s}}(r)$ monotonic and independent of $\phi$ (Cranmer \& Owocki 1996), the deviations from these conditions are not very large, at least for weak DACs, and our assumption seems a reasonable first approximation. Moreover, as we will show, it allows us to make considerable progress with the structure inference problem; without this model assumption the inference of the wind structure is far from trivial (although see Sects. 3.4 and 4.2, where we discuss inferring the structure of general, nonaxisymmetric winds).

The assumption (cf. Point 7 in Sect. 1) that the DAC can be described approximately by a "corotating dense region" or "CDR" with definite radial flow speed $v_{\mathrm{s}}(r)$ independent of $\phi$ and originating at some inner surface is akin to the DAC data analysis modelling by Owocki et al. (1995) and Fullerton et al. (1997) in which, as they put it "the hydrodynamical feedback between density and velocity is ignored".

What the observer sees is then the time-dependent profile of the stream absorption over the range of Doppler speeds presented along the observer line of sight at each moment under the combined effects of radial matter flow and rotation of the matter pattern. It is vital to note that (a): we do not assume the spatial stream flow speed $v_{\mathrm{s}}(r)$ of DAC producing dense matter to be the same as that $\left(v_{\text {wind }}(r)\right)$ of matter in the mean wind producing the overall stellar profile; (b) the apparent pattern/phase speed $v_{\mathrm{p}}$ of the absorption features seen as a function 
of time $(\phi)$ is not the same as that of either $v_{\mathrm{s}}(r)$ nor of $v_{\text {wind }}(r)$ since rotation sweeps matter at larger $r$ into the line of sight. Indeed our approach is aimed at inferring $v_{\mathrm{s}}(r)$ for the stream material and comparing it with the velocity law $v_{\text {wind }}(r)$ of the general wind. It can encompass any monotonic form of velocity law $v_{\mathrm{s}}(r)$, including for example that used for narrow streams by Hamann et al. (2001) with the usual $\beta$-law form plus a superposed inward pattern speed, intended as a qualitative representation of an Abbott wave (Abbott 1980 - cf. Cranmer \& Owocki 1996; Feldmeier \& Shlosman 2002). By not restricting $v_{\mathrm{s}}(r)$ to some parametric form, we should be able to recover, from DAC profile data, information on such features as plateaux in $v_{\mathrm{s}}(r)$, i.e., regions where $v_{\mathrm{s}}^{\prime}(r)$ is small, which can be (see Eq. (1)) at least as important in determining DAC profiles as local density enhancements (Cranmer \& Owocki 1996). By formulating the data diagnostic problem in a non-parametric way in our general treatment (e.g. not enforcing a $\beta$-law) we show that it is possible to infer kinematically the form of $v_{\mathrm{s}}(r)$ and hence the presence both of density enhancements and of velocity plateaux from DAC profile data, and to compare these with dynamical model predictions.

In addition to our model assumption we make the following geometrical and physical idealisations, mostly to clarify the relationship between the intrinsic physics of the wind and its observational characteristics. These simplifications are similar to those used by previous authors in similar regimes of the problem (e.g., Fullerton et al. 1997; Kaper et al. 1999 and references therein).

Firstly, we neglect variations with radius of the ionisation or excitation state of the absorbing ion. Such variations would correspond to an effective source or sink term in the continuity equation (reflecting the fact that the number of absorbers in any fluid element does not remain constant as it moves through the wind), and the function $\mathcal{P}(w)$ in Eq. (6) would consequently be modified. In principle, such variations can be accounted for by analysing lines from a range of ions and levels. This lets us, for example, drop $T(r)$ as an unknown. The absorbing ion density variation with $r$ and $\phi$ is then effectively controlled solely by steady state continuity. We recognise that, in reality, application of continuity to a single ion without allowing for varying ionisation could give very misleading results since variations in ionisation are often observed (Massa et al. 1995; Fullerton et al.1997; Prinja et al. 2002). Determining the ionisation balance throughout the wind - and correcting our continuity equation in light of this - is a separate inference problem that we do not consider here.

Secondly, we make the "point-star" approximation:

1. The system is assumed to be seen at $i=\pi / 2$ and the wind stream structure is approximated as constant (or averaged) across the stellar disk. This essentially reduces the structure problem from 3-D to 2-D, eliminating the spherical coordinate $\theta$.

2. We consider only absorption features formed at large enough distances $r$ compared to the (continuum) stellar radius $R$ so that (a) absorption layers are essentially plane parallel (perpendicular to the line of sight $z$ ) and (b) the line of sight speed of absorbing matter is essentially the radial speed $v_{\mathrm{s}}(r)$ of stream matter away from the star. Though this can hardly apply to the $\mathrm{H}_{\alpha}$ and other Balmer lines, according to Kaper et al. (1999), DACs in the UV are typically not detected until at least $\Delta \lambda / \lambda_{0} \geq 0.2-0.4$, and even higher for Main Sequence stars, so for these our approximations should be reasonable.

3. We assume the time variation of the DAC to arise from the rotation of the perturbation pattern through the line of sight. However we assume that the rotation speed $\Omega(r) r \ll v_{\mathrm{s}}(r)$, where $v_{\mathrm{s}}(r)$ is the radial physical speed of the stream matter, so that the $z$-component of $\Omega(r) r$ does not significantly affect the observed Doppler shifts.

It is a straightforward matter to relate the point-star and finitestar line profiles given the underlying wind density and velocity laws, so this approximation has no significant bearing on the forward modelling of the line-profile variations. The inference of the wind structure from dynamic spectra does have a different character when finite-star effects are considered: the "smearing" of features in the spectrum for a finite stellar disk depends on the wind velocity laws, and therefore to deconvolve this effect to obtain the corresponding point-star spectrum (on which out inversion technique is based) requires, in principle, knowledge of the wind structure that we are hoping to infer. However, this is only important close to the star, and for most of the wind the point-star approximation is adequate.

With the above assumptions we now get, in the Sobolev approximation, that for a transition of oscillator strength $f_{0}$ and rest wavelength $\lambda_{0}$, the optical depth at shift $\Delta \lambda$ is

$$
\begin{aligned}
\tau(\Delta \lambda, \phi) & =\frac{\pi e^{2} f_{0} \lambda_{0}}{m c} \frac{\mathrm{d} N}{\mathrm{~d} v_{\mathrm{s}}} \\
& =\frac{\pi e^{2} f_{0} \lambda_{0}}{m c}\left[\frac{n\left(r\left(v_{\mathrm{s}}\right), \phi\right)}{v_{\mathrm{s}}^{\prime}(r)}\right]_{v_{\mathrm{s}}=\Delta \lambda \mathrm{c} / \lambda_{0}},
\end{aligned}
$$

where $m$ and $e$ are the electron mass and charge, $c$ is the speed of light, and $n, N$ are the values at point $r, \phi$ of the space density and column density to the observer of the ions in the absorbing level. Our assumptions have now resulted in apparently more functional degrees of freedom in the model $\left(n(r, \phi), v_{\mathrm{s}}(r)\right.$ and $\Omega(r))$ than in the data $(\tau(\Delta \lambda, \phi))$, since $\tau$ and $n$ contain the same number of degrees of freedom. It is usual to eliminate one of the remaining degrees of indeterminacy by assuming a form for $\Omega(r)$ (e.g., constant angular momentum) which then leaves us the unknowns $n(r, \phi)$ and $v_{\mathrm{s}}(r)$ apparently involving only one more degree of freedom than the data, which can be removed by recognising the need to satisfy the steady state continuity equation (see below).

In fact, however, we show in Sect. 4.3 that, rather surprisingly, it is not actually necessary to make an assumption on $\Omega(r)$. Due to the separable/self-similar form of the dependence of $\tau$ on $v_{\mathrm{s}}(r), \phi$ and $\Omega(r)$ it proves possible to recover all three functions $v_{\mathrm{s}}, n$ and $\Omega$ from $\tau(\Delta \lambda, \phi)$. This means that $\tau(\Delta \lambda, \phi)$ combined with the continuity equation contain more information than just $n(r, \phi)$ and that we are able to use it to infer not only $n(r, \phi)$ but information on $v_{\mathrm{s}}(r)$ and $\Omega(r)$.

[In the numerical treatment of the DAC data modelling problem by de Jong (2000), the continuity equation is not considered but a form $\Omega \propto r^{-2}$ is adopted and it is further assumed 
that $v_{\mathrm{s}}(r)$ is known (in fact it is taken to be the same as the mean wind speed). For specified $v_{\mathrm{s}}(r)$ they could invert Eq. (1) to get the radial density profile at each $\phi$, viz

$n(r, \phi)=\frac{m c}{\pi e^{2} f \lambda_{0}} v_{\mathrm{s}}^{\prime}(r) \tau\left(v_{\mathrm{s}}(r), \phi\right)$

and so build up a picture of the stream $n(r, \phi)$ structure. However, by specifying $v_{\mathrm{s}}(r)$ and $\Omega(r)$, their approach ignores the steady state continuity equation which the stream material $n(r, \phi), v_{\mathrm{s}}(r)$ and $\Omega(r)$ must satisfy and in fact doubly over-determines the problem since information on both $v_{\mathrm{s}}(r)$ and $\Omega(r)$ is present in $\tau$. In other words, with $v_{\mathrm{s}}(r)$ specified, it may not be possible to find a satisfactory solution of Eq. (2) for $n(r, \phi)$ from DAC data, or to satisfy the continuity equation, unless the adopted $v_{\mathrm{s}}(r)$ and $\Omega(r)$ happen to be in fact the true ones.

The most convenient way to express continuity is to link $n(r, \phi)$ to the stream density $n_{0}\left(\phi_{0}\right)=n\left(R, \phi_{0}\right)$ at some inner boundary surface $r=R$ where the flow speed $v_{\mathrm{s}}(R)=v_{0}$. The original azimuth $\phi_{0}$ of the stream when at $r=R$ is related to its azimuth $\phi$ when in the line of sight at distance $r$ by $\phi=$ $\phi_{0}+\Delta \phi(r)$ where $\Delta \phi(r)$ is determined from $\mathrm{d} \phi=\left(\Omega_{0}-\Omega(r)\right) \mathrm{d} t$ so that

$\Delta \phi=\int_{R}^{r}\left[\Omega_{0}-\Omega(r)\right] \frac{\mathrm{d} r}{v_{\mathrm{s}}(r)}=\int_{v_{0}}^{v}\left[\Omega_{0}-\Omega(r)\right] \frac{\mathrm{d} v_{\mathrm{s}}}{v_{\mathrm{s}} v_{\mathrm{s}}^{\prime}}$.

Here $\Delta \phi(r)$ is the azimuth angle through which a parcel of stream matter has moved between leaving the surface $R$ at point $\phi_{0}$ and reaching distance $r$, or corresponding physical speed $v_{\mathrm{s}}(r)$. If the parcel is in the line of sight when at distance $r$ then $\phi(r)-\phi_{0}=\Delta \phi(r)$.

The continuity equation (see Appendix) then gives

$n(r, \phi)=\frac{v_{0} R^{2}}{v_{\mathrm{s}} r^{2}} n_{0}\left(\phi_{0}=\phi-\Delta \phi(r)\right)$

where we have assumed the flow is 3-D. (If it were strictly 2-D then $v_{0} R^{2} /\left(v_{\mathrm{s}} r^{2}\right)$ would be replaced by $\left.v_{0} R /\left(v_{\mathrm{s}} r\right)\right)$. Thus, once we have determined $n_{0}\left(\phi_{0}\right)$ and $v_{\mathrm{s}}(r)$, we can derive $n(r, \phi)$ everywhere using the continuity Eq. (4).

Before discussing the forward and inverse properties of the problem, we introduce a set of dimensionless variables and parameters:

$$
\begin{array}{ll}
x=r / R ; \quad w=\frac{c \Delta \lambda}{\lambda_{0} v_{\infty}} ; & w_{\mathrm{s}}(x)=v_{\mathrm{s}}(r) / v_{\infty} ; \\
W(x)=\Omega(r) / \Omega_{0} ; & S=\Omega_{0} R / v_{\infty}=V_{\mathrm{eq}} / v_{\infty} ; \\
f(w, \phi)=\tau(\Delta \lambda, \phi) / \tau_{0} ; & \tau_{0}=\frac{\pi e^{2} f_{0} \lambda_{0}}{m c} \frac{n_{0}(0) R}{v_{\infty}} .
\end{array}
$$

Then with $w_{\mathrm{s}}^{\prime}=\mathrm{d} w_{\mathrm{s}} / \mathrm{d} x$ and the important function $\mathcal{P}(w)$ defined by

$\mathcal{P}(w)=\left[\frac{1}{x^{2} w_{\mathrm{s}} w_{\mathrm{s}}^{\prime}}\right]_{w_{\mathrm{s}}=w}$

the dimensionless optical depth equation becomes, using Eqs. (1), (4), and (5),

$f(w, \phi)=\frac{F_{0}(\phi-\Delta \phi(w))}{\left[x^{2} w_{\mathrm{s}} w_{\mathrm{s}}^{\prime}\right]_{w_{\mathrm{s}}=w}}=\mathcal{P}(w) F_{0}(\phi-\Delta \phi(w))$ for dimensionless base density function

$F_{0}\left(\phi_{0}\right)=\frac{n_{0}\left(\phi_{0}\right)}{n_{0}(0)}$

and the stream azimuth shift Eq. (3) becomes

$$
\begin{aligned}
\Delta \phi(w(x)) & =S \int_{1}^{x}[1-W(x)] \frac{\mathrm{d} x}{w_{\mathrm{s}}} \\
& =S \int_{w_{0}}^{w}\left[1-W\left(x\left(w_{\mathrm{s}}\right)\right)\right] \frac{\mathrm{d} w_{\mathrm{s}}}{w_{\mathrm{s}} w_{\mathrm{s}}^{\prime}} .
\end{aligned}
$$

The significance of the function $\mathcal{P}(w)$ derives from the fact that, when the wind density is axisymmetric $\left(F_{0}\left(\phi_{0}\right) \equiv 1\right)$ we have

$f(w, \phi)=\mathcal{P}(w)$,

so that $\mathcal{P}(w)$ is the (time-independent) optical depth profile of the stellar line, in the absence of scattering from the volume of the wind (that is, it is the absorption component of the P Cygni profile).

Using Eq. (7) above we can now consider the DAC diagnostic problem as determining as much as possible about the velocity laws $w_{\mathrm{s}}(x), W(x)$ and the mass loss angular distribution function $F_{0}\left(\phi_{0}\right)$ from optical depth data $f(w, \phi)$ using steady state continuity to reduce the number of degrees of freedom and so make the problem determinate. We will address this from both the forward predictive viewpoint $\left(F_{0}\left(\phi_{0}\right), w_{\mathrm{s}}(x), W(x) \rightarrow f(w, \phi)\right)$ and the inverse deductive one $\left(f(w, \phi) \rightarrow F_{0}\left(\phi_{0}\right), w_{\mathrm{s}}(x), W(x)\right)$.

At this point we note a very important property of expression (7) which is the basis for our later inverse solution of the problem but which also describes the limitation of the purely kinematic model we are using. The time $(\phi)$ evolution of the optical depth line profile function $f(\phi, w)$ is a direct reflection of the azimuthal distribution $F_{0}\left(\phi_{0}\right)$ of the surface mass loss density subject only to a scaling factor $\mathcal{P}(w)$ and a phase shift $\Delta \phi(w)$ wholly determined by the velocity laws $w_{\mathrm{s}}(x), W(x)$. With the $\mathcal{P}(w)$ scaling factor removed the time profile of $f$ should look the same at all $w$ apart from a phase shift. This is a restrictive property of the kinematic model and is not satisfied by $\phi$-periodic functions $f(\phi, w)$ in general. It arises because $F_{0}\left(\phi_{0}\right)$ is time independent and since the kinematic model approximates $w_{\mathrm{s}}=w_{\mathrm{s}}(x)$ only. If $w_{\mathrm{s}}=w_{\mathrm{s}}(x, \phi)$, as in dynamical CIR models where the density stream variation with $\phi$ feeds back on the $w_{\mathrm{s}}=w_{\mathrm{s}}(x, \phi)$, then in general $f(\phi, w)$ will not have the $\mathcal{P}(w)$-scaled, $\Delta \phi(w)$-phase-shifted invariance property we are using here. So, as already noted, we are using a kinematic approximation to the real situation. The extent to which this approximates to dynamical models and to real DAC data is the subject of a future paper but is briefly discussed in Sect. 6 .

We will mainly discuss the problem in terms of general functional forms rather than assumed parametric ones though we will discuss the forward problem in some particular parametric cases of $w_{\mathrm{s}}(x)$ and for $W(x)$ given by the parametric form

$W(x)=x^{-\gamma}$,

where $\gamma=0$ would imply full corotation of a straight stream with the star, $\gamma=2$ is the case usually considered of constant angular momentum (e.g., de Jong 2000; Hamann et al. 2001), 


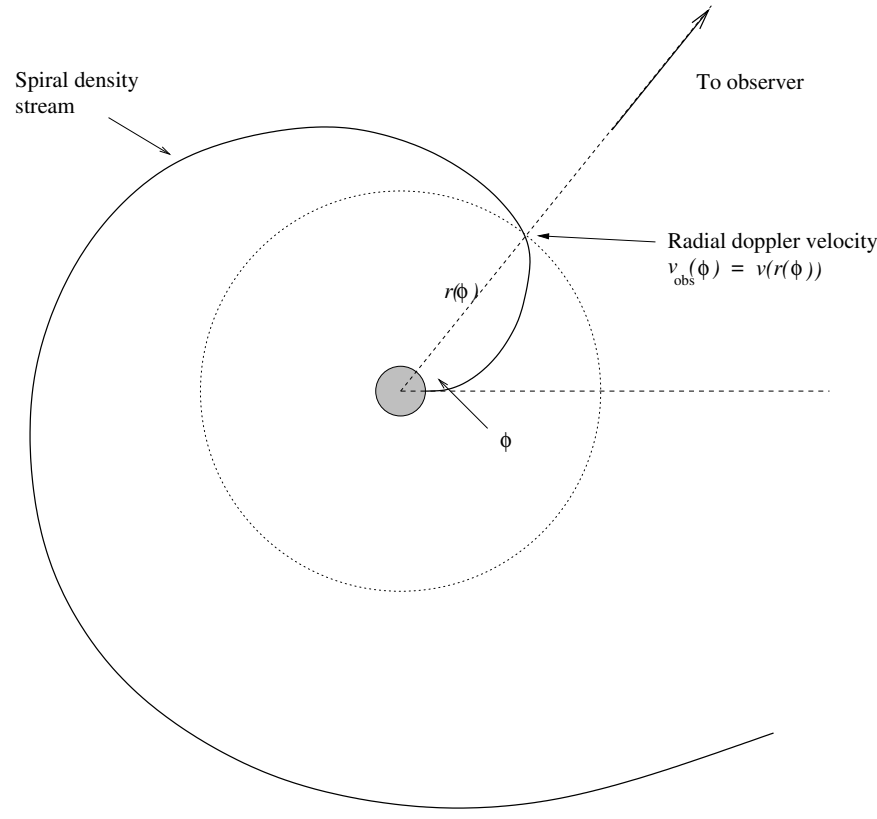

Fig. 1. A schematic illustration of the geometry of a narrow spiral density stream showing that an observer sees a narrow absorption feature corresponding to the radial velocity of the stream material in front of the star at any moment (in the point-star approximation).

and $\gamma \rightarrow \infty$ corresponds to rapid damping of the angular momentum with distance, stream matter moving in purely radial lines in the observer frame.

\section{Properties of narrow streams}

\subsection{Predicted DAC properties for general $w_{\mathrm{s}}(x)$ and $W(x)$ laws}

By a narrow stream we mean one in which the dense outflow at the inner boundary is not very extended in azimuth so that the spread in consequent DAC $\Delta \lambda \ll$ the overall width of the absorption line.

This results in a narrow range of stream $x$ at each $\phi$ and so in a narrow range $(\ll 1)$ of $w(x)$ in the line of sight at any given time $\phi$ (see Fig. 1). In the limiting case we can describe this by $F_{0}\left(\phi_{0}\right) \propto \delta\left(\phi_{0}\right)$ where $\delta\left(\phi_{0}\right)$ is the delta function and we arbitrarily adopt the mass loss point as $\phi_{0}=0$. It is obvious physically and from Eq. (1) that at any observer azimuth $\phi$ (i.e., time) the DAC will appear as a sharp feature in $f$ at a single Doppler shift $w$ (Fig. 1). Although the stream density pattern is time independent in the stellar frame, it is carried by rotation across the line of sight as shown in Fig. 1 and so the Doppler shift changes with $\phi$ and at a rate determined by the stream geometry as well as by the physical flow speed $w_{\mathrm{s}}(x)$ of the stream matter. We describe this in more detail in Sect. 3.4.1. What the observer sees is an acceleration due to the changing view angle of the density pattern and we will use the terms "pattern speed $w_{\mathrm{p}}$ " and "pattern acceleration $a_{\mathrm{p}}$ " for this (cf. Fullerton et al. 1997; Hamann et al. 2001). To find the value $w$ of this Doppler shift speed as a function of "time" $\phi$ we equate the observer direction $\phi$ to the angle $\phi_{\mathrm{p}}$ at which the dense stream matter passing through the line of sight at that time has spatial speed $w$.

Since we have adopted $\phi_{0}=0$ as the stream base point this yields, using Eq. (3) and recalling that $\phi$ is measured in the corotating stellar frame,

$$
\begin{aligned}
\Omega_{0} t=\phi_{\mathrm{p}}(w) & =\phi_{0}+\Delta \phi(w)=\Delta \phi(w) \\
& =S \int_{w_{0}}^{w}\left[1-W\left(x\left(w_{\mathrm{s}}\right)\right)\right] \frac{\mathrm{d} w_{\mathrm{s}}}{w_{\mathrm{s}} w_{\mathrm{s}}^{\prime}} .
\end{aligned}
$$

Thus $\phi_{\mathrm{p}}(w)$ defines the pattern (or phase) speed $w_{\mathrm{p}}(\phi)$ with which the matter appears to accelerate as the outflowing stream is rotated through the line of sight and does not give the actual speed variation of any particular part of the structure.

Expression (12) is valid for any form of $W, w_{\mathrm{s}}$ and, as first noted by Hamann et al. (2001) for specific $W, w_{\mathrm{s}}$, reveals a surprising and important property of such kinematic DAC models which appears to have received little mention hitherto though the essential equations are contained in e.g., Fullerton et al. (1997). $\phi=\Omega_{0} t$ is a measure of time so $\phi_{\mathrm{p}}$ in Eq. (12) measures the time it takes the DAC produced by the rotating pattern to accelerate (apparently) from $w_{0} \approx 0$ to $w$-i.e., for the observer to rotate from $\phi=\phi_{0}=0$ to the azimuth where matter in the line of sight has speed $w$. This can be compared with the "time" $\phi_{\mathrm{s}}$ it takes for a single actual element of stream matter moving with the same radial flow speed law $w_{\mathrm{s}}(x)$, to accelerate from $w_{0}$ to $w$, namely

$\phi_{\mathrm{s}}(w)=S \int_{w_{0}}^{w} \frac{\mathrm{d} w_{\mathrm{s}}}{w_{\mathrm{s}} w_{\mathrm{s}}^{\prime}}$.

In the terminology of Prinja \& Howarth (1988) this would be termed the behaviour of a "puff".

Comparison of Eqs. (12) and (13) immediately shows that for any rotation law satisfying $W(x)=\Omega(r) / \Omega_{0}>0$ (which is the case for all plausible $\Omega$ )

$\phi_{\mathrm{p}}(w)<\phi_{\mathrm{s}}(w)$

(this follows because $w_{\mathrm{s}} w_{\mathrm{s}}^{\prime}>0$, so the $W(x)$ term in Eq. (12) always decreases the integrand).

This generalises the result of Hamann et al. (2001) and is surprising in two ways. First the DAC seen from a corotating structure in which the matter follows a radial flow speed law $w_{\mathrm{s}}(x)$ takes less time to reach (i.e., accelerates faster up to) any observed Doppler speed $w$ than would an absorption feature produced by a transient puff of material following the same flow law $w_{\mathrm{s}}(x)$. Second, the enhancement of the apparent acceleration of the DAC from the corotating stream pattern over that for the puff is independent of the absolute value $\Omega_{0}$ of the rotation rate (though it does depend on the relative variation $W(x)$ of $\Omega$ with $x)$. This can also be expressed in terms of $d i$ mensionless accelerations

$a=\frac{R}{v_{\infty}^{2}} \frac{\mathrm{d} v}{\mathrm{~d} t}=\frac{R}{v_{\infty}^{2}} \Omega_{0} \frac{\mathrm{d} v}{\mathrm{~d} \phi}=S \frac{\mathrm{d} w}{\mathrm{~d} \phi}=\frac{S}{\mathrm{~d} \phi / \mathrm{d} w}$.

Evaluating $\mathrm{d} \phi / \mathrm{d} w$ for $\phi_{\mathrm{p}}(w)$ and $\phi_{\mathrm{s}}(w)$ given by Eqs. (12) and (13) we obtain the actual, physical acceleration of stream matter (equal to that which would be observed for absorption in a puff):

$a_{\mathrm{s}}(w)=\left[w_{\mathrm{s}} w_{\mathrm{s}}^{\prime}\right]_{w_{\mathrm{s}}=w}$ 
(which is trivial), and the apparent acceleration for the pattern Doppler shift $w$ of a DAC from a rotating spiral density stream

$a_{\mathrm{p}}(w)=\left[\frac{w_{\mathrm{s}} w_{\mathrm{s}}^{\prime}}{1-W\left(x\left(w_{\mathrm{s}}\right)\right)}\right]_{w_{\mathrm{s}}=w}=\frac{S}{\phi_{\mathrm{p}}^{\prime}}$

so that

$\frac{a_{\mathrm{p}}(w)}{a_{\mathrm{s}}(w)}=\frac{1}{1-W\left(x\left(w_{\mathrm{s}}=w\right)\right)}>1$

(for $W(x)>0$ ).

These results are rather counter-intuitive. It is tempting to think that DACs are observed to accelerate more slowly than the mean wind because the long rotation period of the star (compared with wind flow time $R / v_{\infty}$ ) carries the absorbing stream across the line of sight only slowly. In fact Eq. (18) shows that precisely the opposite is true. As time passes any rotation brings into the line of sight stream matter which left the star progressively earlier. This increases the rate at which higher Doppler speeds are seen above the rate due to material motion alone (which is the rate exhibited by a puff of the same material speed) - that is, $a_{\mathrm{p}}$ is the phase acceleration of a pattern (cf. Hamann et al. 2001). This is very important because it means that, at least for narrow streams (but see also Sect. 4.1), for the slow observed acceleration $a_{\mathrm{p}}$ of DACs (compared to the mean wind acceleration $a_{\text {wind }}$ ) to be attributed to a corotating density pattern the actual flow acceleration $a_{\mathrm{s}}$ of the matter creating that pattern must be lower than $a_{\text {wind }}$ since the apparent $a_{\mathrm{p}}$ is in fact higher than the physical acceleration $a_{\mathrm{s}}$ of the stream matter. That is, for the observed DAC (pattern) acceleration to be slow compared with the mean wind, the stream matter acceleration must be very slow compared with the wind. For example, Hamann et al. (2001), addressing the forward problem, added a constant inward speed plateau to the general outflow to represent empirically the presence of an Abbott wave (Abbott 1980). In the dynamical modelling results of Cranmer \& Owocki (1996), denser material is accelerated more slowly because of its greater inertia per unit volume. This lends motivation to our aim of providing a means of inferring the true flow speed of dense stream matter direct from recurrent DAC data. The result may also provide a partial explanation for why de Jong (2000) found difficulty in fitting data with a parametric stream density model $n(r, \phi)$ since they assumed a flow speed $w_{\mathrm{s}}(x)$ equal to that of the mean wind. Such a flow speed model should, from the above results, predict apparent DAC (pattern) accelerations higher than those of the mean wind and so could never properly fit the observed slow accelerations. (Recall also that de Jong 2000 did not ensure that their $n(r, \phi)$, $v(r), W(r)$ satisfied the continuity equation.)

The second surprise, that $a_{\mathrm{p}}$ is independent of the absolute rotation rate $S$, can be understood by the fact that although higher $\Omega_{0}$ sweeps the dense matter pattern across the line of sight faster, the pattern itself is more curved for higher $\Omega_{0}$. The effects of higher rate and of greater stream curvature cancel out. It is also instructive to note the two limiting cases of $W=\Omega / \Omega_{0}$. For $W \rightarrow 0(\gamma \rightarrow \infty)$ we get $a_{\mathrm{p}}(w)=a_{\mathrm{s}}(w)$ because all stream elements observed are moving directly toward the observer, and for $W \rightarrow 1,(\gamma \rightarrow 0) a_{\mathrm{p}}(w) / a_{\mathrm{s}}(w) \rightarrow \infty$ because the density stream is straight and radial and all points $(w)$ along it are swept into the line of sight at the same moment.

The finding that the ratio of the observed apparent stream pattern acceleration $a_{\mathrm{p}}$ compared with the true matter acceleration should be independent of $\Omega_{0}$ does not contradict the data (Kaper et al. 1999) which suggest a correlation between observed acceleration and $\Omega_{0}$. This is because (see Fig. 5, Sect. 4.1) the translation from data on $w(\phi)$ to $w_{\mathrm{s}}(x)$ involves the value of $S$. In addition, only a wide-stream analysis is adequate fully to describe the situation, since the acceleration of the peak of a DAC from a wide stream depends on the density function $F_{0}\left(\phi_{0}\right)$ which may be affected by the rotation rate $\Omega_{0}-$ see Sect. 4.2.2.

\subsection{Explicit expressions for $\beta$-law parametric form of $w_{\mathrm{s}}(x)$}

Though we are mainly seeking to address the DAC problem non-parametrically, explicit expressions for some of the results in Sect. 3.1 for particular forms of $w(x)$ are useful for illustrating properties of the kinematic DAC model such as the dependence of streak and stream line shape on rotation and acceleration parameters (e.g., $\beta, \gamma$ ).

Here, for reference, we restate some results of Hamann et al. (2001) for the $\beta$-law (with $w_{0}=0$ )

$w_{\mathrm{s}}(x)=\left(1-\frac{1}{x}\right)^{\beta}$

For this $\beta$-law and for form Eq. (11) of the rotation $W(x)$ law we obtain the following expressions

$\Delta \phi(w)=S \int_{1}^{x(w)}\left(1-\frac{1}{x^{\gamma}}\right) \frac{\mathrm{d} x}{w_{\mathrm{s}}(x)}$

$\left(x(w)=\left(1-w^{1 / \beta}\right)^{-1}\right)$. In general Eq. (20) has to be evaluated numerically, which is rather inconvenient, especially if (cf. below) one wants to invert to get $w(\phi)$, but the integration is analytic for some specific cases (so long as we approximate $w_{0}=0$ ). In particular for constant stream angular momentum $(\gamma=2)$ with $\beta=1 / 2$ we get

$\frac{\phi_{\mathrm{p}}(w)}{S}=\frac{1}{2} \log \left(\frac{1+w}{1-w}\right)+\frac{w}{1-w^{2}}-2 w$,

while the time $\phi_{\mathrm{s}}$ at which a puff moving radially with speed $w_{\mathrm{s}}(x)$ would reach speed $w$ is just the first two terms of the the above integral, i.e.,

$\frac{\phi_{\mathrm{s}}}{S}=\frac{1}{2} \log \left(\frac{1+w}{1-w}\right)+\frac{w}{1-w^{2}}$.

For $\beta=1$ we get

$\frac{\phi_{\mathrm{p}}(w)}{S}=\frac{w}{1-w}-\log (1-w)$,

and

$\frac{\phi_{\mathrm{s}}(w)}{S} \sim \frac{w}{1-w}+\log \left(\frac{w}{1-w}\right)$

(a logarithmically divergent term $\log w_{0} \rightarrow-\infty$ has been neglected in Eq. (24): this simply indicates that the $\beta=1$ velocity 
law is singular at the stellar surface; fluid elements take an infinitely long time to accelerate to a finite velocity).

Note that for arbitrary $\beta$, with $\gamma \rightarrow \infty$ (no stream angular momentum) the second term in the integrand in Eq. (20) vanishes and stream material moves purely radially, rotation serving only to "time-tag" the part of the stream in the observer line of sight. The observed stream Doppler speed is then just the actual matter speed and $\phi_{\mathrm{p}}(w)=\phi_{\mathrm{s}}(w)$ for all $w$.

Secondly, for arbitrary $\beta$, with $\gamma=0$ (rigid stream corotation), stream matter corotates rigidly with the star and all points along it are seen simultaneously, corresponding to infinite apparent acceleration or $\phi_{\mathrm{p}}(w)=0$ for all $w$.

\subsection{A new $\alpha$-law parametrisation of $w(x)$}

Though we do not use it explicitly in the present paper we suggest here a new parametric form of velocity law which should prove useful in future studies of hot-star winds, particularly from an inferential point of view, as it makes it easier to obtain analytic results. This "alpha-law" parametric form for $w_{\mathrm{s}}(x)$, in contrast to the $\beta$-law, allows exact analytic integration to give $\phi_{\mathrm{p}}(w)$ and $\phi_{\mathrm{s}}(w)$ for any value of a continuously variable acceleration parameter $\alpha$ and for any finite $w_{0}$, namely

$w_{\mathbf{s}}(x)=\frac{1}{1+\frac{1-w_{0}}{w_{0}} x^{-\alpha}}$.

Note that for this form it is essential to retain non-zero $w_{0}$. This has the required asymptotic values $w_{\mathrm{s}}(1)=w_{0}, w_{\mathrm{s}}(\infty)=1$ and $\alpha$ parametrises a "typical" acceleration just as $\beta$ does for $\beta$-law Eq. (19). This $\alpha$-law form has no physical basis but nor for that matter do $\beta$-laws other than the one special (CAK) case $\beta=1 / 2$. However, $\beta$-laws are so widely used as a way of fitting DAC and wind data (to the point that they are often thought of as "reality") that it is interesting to see how $\alpha$-laws compare with them. In Fig. 2 we have plotted $\beta$-law $w_{\mathrm{s}}(x)$ for $\beta=\frac{1}{2}$, 1 and 2 and for $w_{0}=0.1$ together with "eyeball" $\alpha$-law best matches to each with the "best" value of $\alpha$ indicated. It is clear that the $\alpha$-laws resemble the $\beta$-laws quite well within the typical uncertainties of data and models. The match is least good at small $x$ and $w_{\mathrm{s}}$ values and is worse for smaller values of $w_{0}$ where in any case data are very sparse (Kaper et al. 1999), the physics is least certain, and to which the point-star approximation (Sect. 2) does not apply in any case.

\subsubsection{Explicit expressions for $\alpha$-law $w_{\mathrm{s}}(x)$}

Using Eq. (25) we obtain the following expressions

$$
\begin{aligned}
& x\left(w_{\mathrm{s}}\right)=\left[\frac{w_{\mathrm{s}}}{w_{0}} \frac{1-w_{0}}{1-w_{\mathrm{s}}}\right]^{\frac{1}{\alpha}} \\
& w_{\mathrm{s}} w_{\mathrm{s}}^{\prime}=\alpha\left(\frac{w_{0}}{1-w_{0}}\right)^{\frac{1}{\alpha}} \frac{\left(1-w_{\mathrm{s}}\right)^{1 / \alpha+1}}{w_{\mathrm{s}}^{1 / \alpha-1}} \\
& x^{\gamma} w_{\mathrm{s}} w_{\mathrm{s}}^{\prime}=\alpha\left(\frac{1-w_{0}}{w_{0}}\right)^{\frac{\gamma-1}{\alpha}} \frac{w_{\mathrm{s}}^{(\gamma+\alpha-1) / \alpha}}{\left(1-w_{\mathrm{s}}\right)^{(\gamma-\alpha-1) / \alpha}} .
\end{aligned}
$$

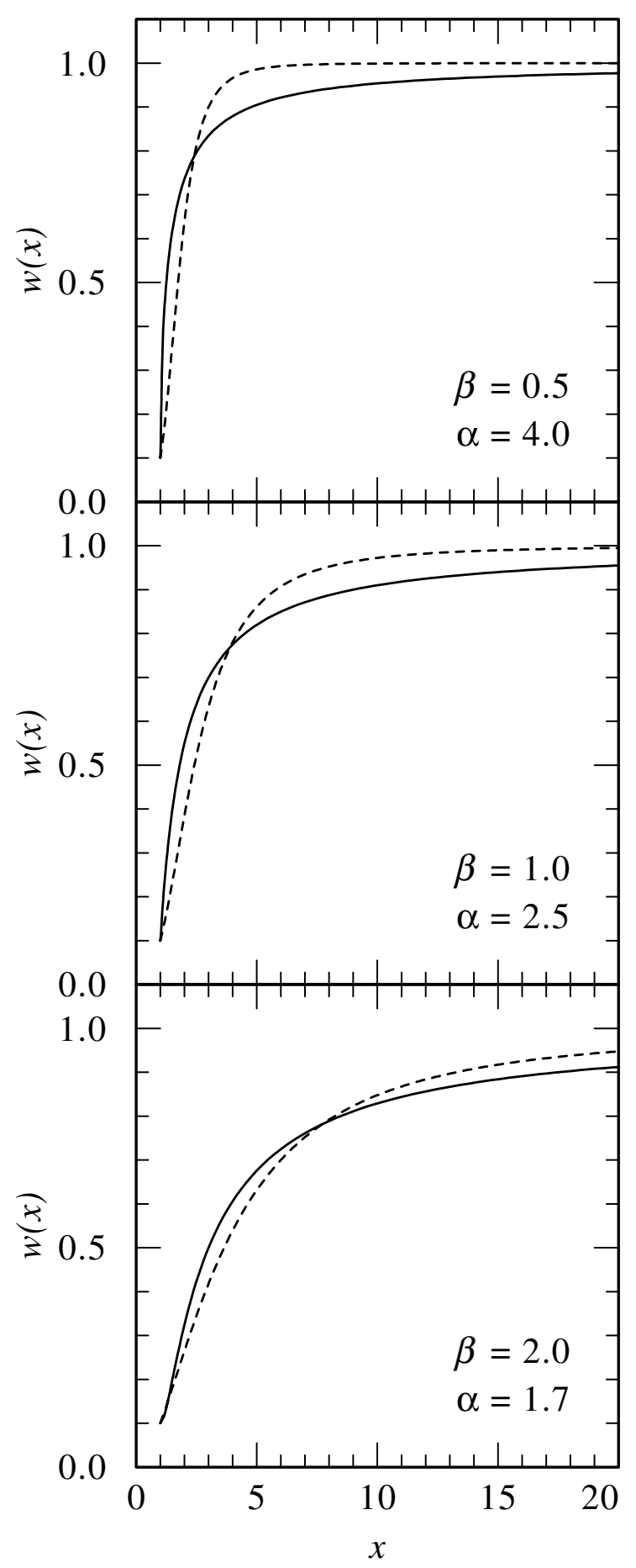

Fig. 2. Comparison of approximate best fit $\alpha$-law parametric forms of $w(x)$ with $\beta$-law $w(x)$ (Eqs. (19) and (25)) for $w_{0}=0.1$. Solid lines represent $\alpha$-law and dotted lines represent $\beta$-law. Parameters $\alpha$ and $\beta$ are indicated within each panel.

Then for the times $\phi$ to reach speed $w$ in the case of a puff and of a rotating pattern we obtain

$\phi_{\mathrm{p}}\left(w_{\mathrm{s}}\right)=x\left(w_{\mathrm{s}}\right)-1+\frac{1}{\alpha-1} \frac{1-w_{0}}{w_{0}}\left[1-x\left(w_{\mathrm{s}}\right)^{-\alpha+1}\right]$ 
and

$$
\begin{aligned}
\phi_{\mathrm{p}}(w)= & x\left(w_{\mathrm{s}}\right)-1+\frac{1}{\alpha-1} \frac{1-w_{0}}{w_{0}}\left[1-x\left(w_{\mathrm{s}}\right)^{-\alpha+1}\right] \\
& -\frac{1}{\gamma-1}\left[1-x^{-\gamma+1}\left(w_{\mathrm{s}}\right)\right] \\
& -\frac{1}{\alpha+\gamma-1}\left[1-x\left(w_{\mathrm{s}}\right)^{-\alpha-\gamma+1}\right]
\end{aligned}
$$

where $x\left(w_{\mathrm{s}}\right)$ is given by Eq. (26).

\subsection{Inversion to find stream flow speed $w_{\mathrm{s}}(x)$ from observed DAC pattern speed $w_{\mathrm{p}}(\phi)$ for a narrow stream}

We have seen in Sect. 3.1 that the actual stream matter flow acceleration $a_{\mathrm{s}}(\phi)$ in a corotating density pattern must be slower than the apparent (pattern) acceleration $a_{\mathrm{p}}(\phi)$ (and much slower than typical wind acceleration $a_{\text {wind }}$ ) in order to match typical narrow DAC observations - cf. results in Hamann et al. (2001) for $\beta$-laws. More generally it is of interest to see whether it is possible to infer the actual flow speed $w_{\mathrm{s}}(x)$ from sufficiently good data on the apparent DAC acceleration. We do so here assuming $W(x)$ is known. What we observe is a pattern speed $w_{\mathrm{p}}(\phi)$ as a function of time $\phi / \Omega_{0}$. What we really want is the true matter flow speed law $w_{\mathrm{s}}(x)$

\subsubsection{Forward problem}

The forward problem is to determine how the observed lineprofile variations are determined by the physical properties of the stream, i.e., to find the observed $w_{\mathrm{p}}(\phi)$ given the wind law $w_{\mathrm{s}}(x)$. This is illustrated in Fig. 3. The wind velocity and rotation laws $w_{\mathrm{s}}(x)$ and $W(x)$ give the physical velocity of fluid elements in the stream (in the corotating frame), from which we can determine the shape of the matter spiral (i.e., the streamline of the CDR): $\mathrm{d} x / \mathrm{d} t=v(r) / R=\left(v_{\infty} / R\right) w_{\mathrm{s}}(x)$ and $\mathrm{d} \phi / \mathrm{d} t=$ $\Omega-\Omega_{0}=\Omega_{0}[1-W(x)]$, so that

$\frac{\mathrm{d} x}{\mathrm{~d} \phi}=\frac{\mathrm{d} x}{\mathrm{~d} t} / \frac{\mathrm{d} \phi}{\mathrm{d} t}=\frac{1}{S} \cdot \frac{w_{\mathrm{s}}(x)}{1-W(x)}$

( $\left.S=\Omega_{0} R / v_{\infty}\right)$, which can be integrated to give $\phi(x)=\Delta \phi(x)$ (see Eq. (3)). This is monotonic, by assumption $(W(x) \leq 1$ ), and so can be inverted (in general numerically) to obtain the spiral law $x(\phi)$. Once we have the spiral law for the streamline of the CDR we can use the (monotonic) velocity law $w_{\mathrm{s}}(x)$ to describe the spiral in terms of the variation of radial velocity with $\phi$, as shown in Fig. 3:

$w_{\mathrm{p}}(\phi)=w_{\mathrm{s}}(x(\phi))$

as was done for $\beta$-laws in Hamann et al. (2001). This $w_{\mathrm{p}}(\phi)$ is the actual doppler velocity that is observed when matter at $\phi$ is in front of the star, so unwrapping the velocity spiral $w_{\mathrm{p}}(\phi)$ directly gives the observed dynamic spectrum, as shown Fig. 3.

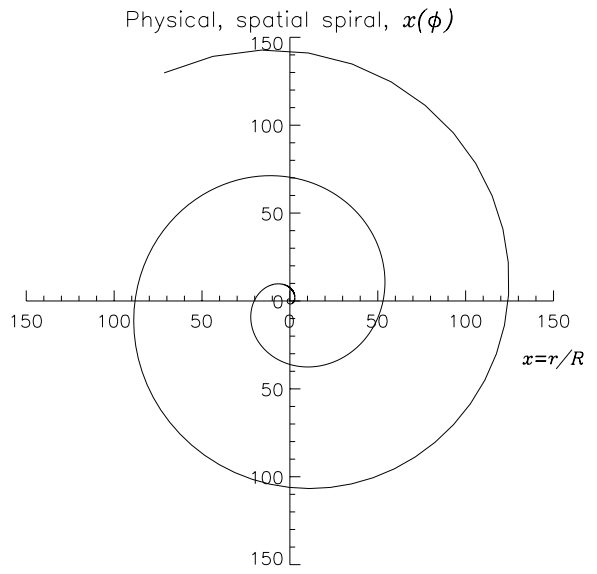

transform $x$ to doppler $\downarrow$ velocity using $v(r)$-law
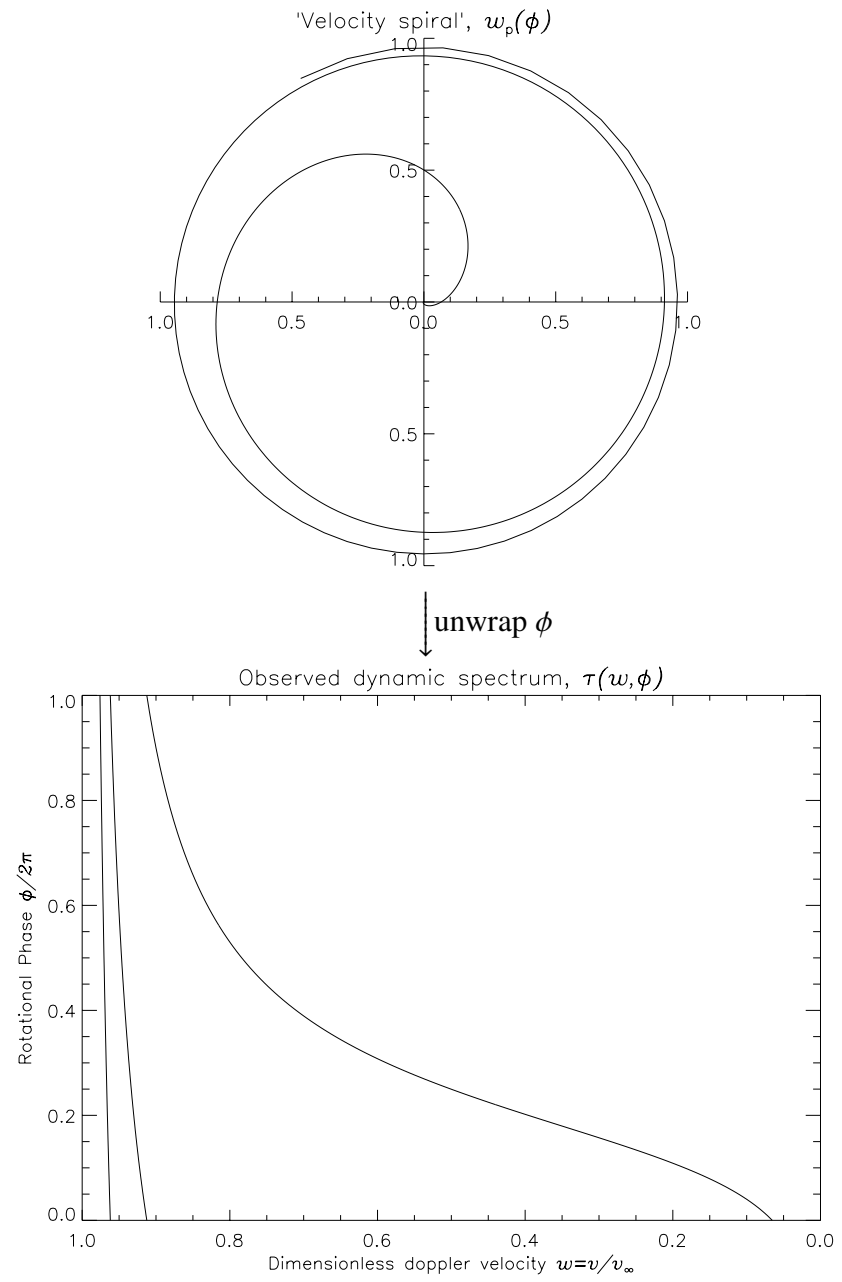

Fig. 3. Obtaining the narrow-stream DAC dynamic spectrum $\tau(w, \phi)$ for given $v(r)$ and $\Omega(r)$ : i) $v$ and $\Omega$ fix the shape of the physical CDR spiral $x(\phi)$ in the corotating frame (see text); ii) converting radius to radial velocity gives the "velocity spiral" $w_{\mathrm{p}}(\phi)$; iii) unwrapping $\phi$ gives $\tau(w, \phi)$, showing how the position of the absorption feature varies as a function of rotational phase (the three lines correspond to successive windings of the spiral - see Fig. 1). Note that for the narrow-stream inversion we only use the position of the absorption feature in the spectrum, $w_{\mathrm{p}}(\phi)$, not the actual value of the optical depth. Figures are for a $\beta=4$ law with constant angular momentum $(\gamma=2)$. 


\subsubsection{Narrow-stream inversion given $\Omega(r)$}

The inverse problem is to find $w_{\mathrm{s}}(x)$ given observations of the time-dependent Doppler shift in the form of the monotonic "velocity spiral" function

$w(\phi)=w_{\mathrm{p}}(\phi) ; \quad$ or $\phi=\phi(w)$.

Here we present the solution to this inverse problem when we assume that we know the rotation law $\Omega(r)$. In this case, the observed line profile variations contain enough information to determine $w_{\mathrm{s}}(x)$ without the need to use the continuity equation. This fact has important consequences for the significance of the inversion, as we discuss below.

In order to recover $w_{\mathrm{s}}(x)$ we need to determine the spatial spiral law $x(\phi)$ (see Fig. 3), because then we will know, at any $\phi$, the distance of the absorbing material from the star and its radial velocity $w_{\mathrm{p}}(\phi)$, which immediately gives us the wind velocity law. In other words we need to translate $w(\phi)$ from the observed time variable $\phi$ to the real spatial variable $x=x_{\mathrm{s}}$ defining the distance at which the absorbing matter lies when it has speed $w$.

This translation is most easily achieved as follows. Imagine we did not have the CIR (or CDR) model of the narrow DAC feature, but instead thought that the DAC results from a spherical shell of material emitted from the star at some instant (a "puff"). Then at each time the $w_{\mathrm{p}}$ we observe would be the radial velocity of this shell as it accelerates through the wind. Assuming that the shell is ejected from the surface, $x=1$, we can integrate up $\mathrm{d} x / \mathrm{d} t=w_{\mathrm{p}}$ to obtain the actual spatial position $x_{\mathrm{p}}(t)$ of the shell at each time:

$x_{\mathrm{p}}(\phi)=1+\frac{1}{S} \int_{0}^{\phi} w_{\mathrm{p}}\left(\phi^{\prime}\right) \mathrm{d} \phi^{\prime}$,

(where, as usual, we have chosen the time unit to be related to the rotation period of the star, so that $t=\phi$; the $1 / S$ factor depending on the rotation rate appears as a result of this conversion from $t$ to $\phi$ in the integral, not for any physical reason see Eq. (5)). Thus, $x_{\mathrm{p}}$ is a notional distance which would be reached by a particle actually moving with the observed apparent pattern speed $w_{\mathrm{p}}(\phi)$. Since $w_{\mathrm{p}}>0, x_{\mathrm{p}}(\phi)$ is monotonic and the result of Eq. (34) can be inverted to yield $\phi\left(x_{\mathrm{p}}\right)$ and hence from Eq. (33)

$w\left(x_{\mathrm{p}}\right)=w_{\mathrm{p}}\left(\phi\left(x_{\mathrm{p}}\right)\right)$.

This is then the wind law that would be derived if we believed the DAC resulted from a puff of material.

However, we really believe that the observed DAC results from a CIR or CDR and, whereas the puff is a time-dependent, axisymmetric disturbance in the wind density, the CDR spiral is a stationary (in the corotating frame), nonaxisymmetric density perturbation. As a result, the observed DAC feature does not directly trace the actual motion of matter through the wind, but rather reflects the shape of the spiral pattern. As the star rotates (i.e., as $\phi$ increases) we see fluid elements at parts of the spiral further out in the wind $(x(\phi)$ increases), where the wind velocity $w_{\mathrm{s}}(x)$ is larger: effectively, the velocity of the actual material doesn't increase as fast as that of the spiral itself (see the discussion of DAC acceleration in Sect. 3.1, after Eq. (18)). We must somehow account for the fact that we do not see the same (or, at least, equivalent) fluid elements at different times.

In order to understand the relationship between the material velocity and the "spiral" velocity we must stop identifying time with $\phi$, because the difference between the puff and CDR interpretations derives precisely from the difference between the way material moves in time and the spiral moves in $\phi$. If at time $t$ the absorbing material in the spiral is at $\phi(t)=\Omega_{0} t$ and $x_{\mathrm{s}}=x(\phi(t))$ (and so has velocity $w_{\mathrm{s}}\left(x_{\mathrm{s}}\right)$ ), after a short time $d t$ this fluid element will have moved through a distance

$\mathrm{d} x_{\mathrm{p}}=\frac{v_{\infty}}{R} w_{\mathrm{s}}\left(x_{\mathrm{s}}\right) \mathrm{d} t$

exactly as for the puff model. However, we will now be seeing absorption from another fluid element in a different part of the spiral, at $\phi=\Omega_{0}(t+\mathrm{d} t)$, with position $x\left(\phi+\Omega_{0} t\right)=$ $x_{\mathrm{s}}+(\mathrm{d} x / \mathrm{d} \phi) \Omega_{0} \mathrm{~d} t$. So the actual change in radius of the spiral is

$$
\begin{aligned}
\mathrm{d} x_{\mathrm{s}} & =\left.\frac{\mathrm{d} x}{\mathrm{~d} \phi}\right|_{x_{\mathrm{s}}} \Omega_{0} \mathrm{~d} t=\left(\frac{\mathrm{d} x}{\mathrm{~d} t} / \frac{\mathrm{d} \phi}{\mathrm{d} t}\right) \Omega_{0} \mathrm{~d} t \\
& =\frac{v_{\infty}}{R} \cdot \frac{w_{\mathrm{s}}\left(x_{\mathrm{s}}\right)}{1-W\left(x_{\mathrm{s}}\right)} \mathrm{d} t
\end{aligned}
$$

Now, we want to determine $x_{\mathrm{s}}$, which is the physical spiral radius, but we cannot as it stands because $\mathrm{d} x_{\mathrm{s}} / \mathrm{d} t$ from Eq. (37) depends on $w_{\mathrm{s}}$, which we don't know. We do, however, know $\mathrm{d} x_{\mathrm{p}}$ in Eq. (36), because we obtained $x_{\mathrm{p}}$ directly from the observed $w_{\mathrm{p}}(\phi)$ in Eq. (34), and we can relate $x_{\mathrm{s}}$ to $x_{\mathrm{p}}$ through

$\frac{\mathrm{d} x_{\mathrm{p}}}{\mathrm{d} x_{\mathrm{s}}}=\frac{\mathrm{d} x_{\mathrm{p}}}{\mathrm{d} t} / \frac{\mathrm{d} x_{\mathrm{s}}}{\mathrm{d} t}=w_{\mathrm{s}}\left(x_{\mathrm{s}}\right) \frac{1-W\left(x_{\mathrm{s}}\right)}{w_{\mathrm{s}}\left(x_{\mathrm{s}}\right)}=1-W\left(x_{\mathrm{s}}\right)$.

Since this only depends on the given rotation law $W(x)$ we can integrate $\mathrm{d} x_{\mathrm{p}} / \mathrm{d} x_{\mathrm{s}}$, again assuming $x_{\mathrm{p}}=1$ at $x_{\mathrm{s}}=1$, to obtain

$x_{\mathrm{p}}\left(x_{\mathrm{s}}\right)=1+\int_{1}^{x_{\mathrm{s}}}\left[1-W\left(x^{\prime}\right)\right] \mathrm{d} x^{\prime}$.

Inserting this into Eq. (35) gives the solution we seek:

$w_{\mathrm{s}}\left(x_{\mathrm{s}}\right)=w\left(\phi\left(x_{\mathrm{p}}\left(x_{\mathrm{s}}\right)\right)\right)$.

To illustrate this, suppose we observe a Doppler shift variation $w(\phi)$ of the form which would arise if we were seeing absorption by a puff of matter moving toward us with a $\beta=1$ velocity law. From Eq. (22) this would produce

$\phi(w)=S\left[\log \left(\frac{w}{1-w}\right)+\frac{1}{(1-w)}\right]$.

We have computed $\phi(w)$ from Eq. (41) for $S=0.1$ and inverted numerically to get $w_{\mathrm{p}}(\phi)$ then used Eq. (34) to get $x_{\mathrm{p}}(\phi)$. To translate $x_{\mathrm{p}} \rightarrow x_{\mathrm{s}}$ we use the particular case $W=1 / x^{2}$ for which Eq. (39) gives

$x_{\mathrm{p}}\left(x_{\mathrm{s}}\right)=x_{\mathrm{s}}+\frac{1}{x_{\mathrm{s}}}-1$

or

$x_{\mathrm{s}}\left(x_{\mathrm{p}}\right)=\frac{1+x_{\mathrm{p}}+\left[\left(1+x_{\mathrm{p}}\right)^{2}-4\right]^{\frac{1}{2}}}{2}$. 


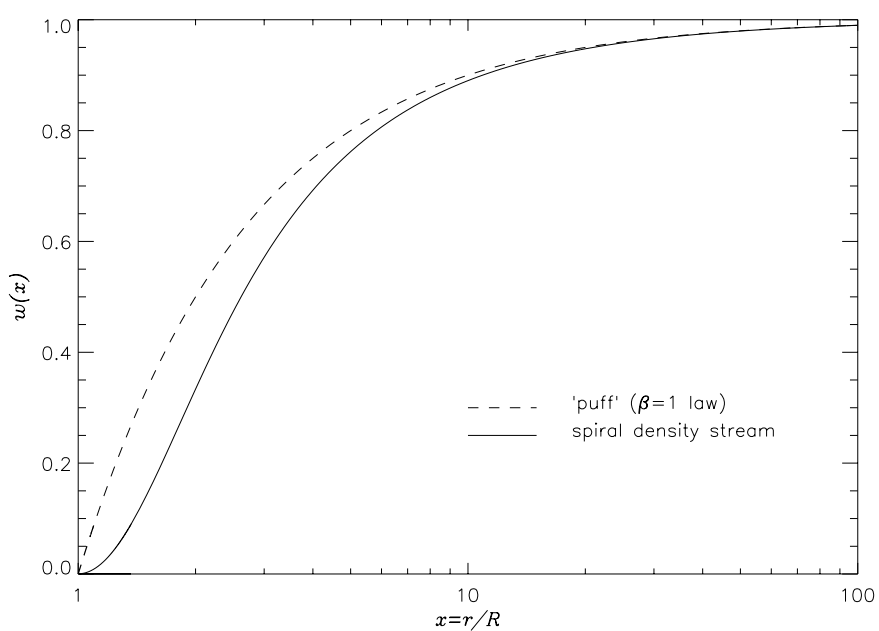

Fig. 4. Solution of the narrow-stream velocity inversion problem. The upper curve is an apparent Doppler shift law $w_{\mathrm{p}}(x)$ law, equivalent to that from a moving "puff" following a $\beta=1$ law. The lower curve shows the inverse solution of the rotating narrow stream problem, namely the stream matter velocity law $w_{\mathrm{s}}(x)$ which would be required to produce the same observed Doppler shift as a function of time when rotation is included.

In Fig. 4 we show the resulting actual matter speed $w_{\mathrm{s}}(x)$ required for a rotating stream to give the same observed $w(\phi)$ as that from a $\beta=1$ law puff motion. In line with our earlier discussion the resulting $w_{\mathrm{s}}(x)$ has a slower acceleration than the $\beta=1$ law, looking more like a $\beta=1.5$ law. These results show just how important it is to include the effect of pattern rotation when interpreting apparent DAC accelerations.

It will pay to think a little more deeply about the narrowstream inversion procedure we have just set out. At no stage did we make use of the value of the optical depth along the streamline, only the doppler velocity at which the absorption occurs. It is for this reason that the continuity equation is not required (and, indeed, cannot be used as a constraint). Furthermore, we required knowledge only of the fluid flow along the single CDR streamline of the narrow DAC, not of any neighbouring streamlines. Most importantly, we did not directly use the assumption that the wind velocity is axisymmetric: the velocity law that we derive does not depend on our model assumption of Sect. 2. The only sense in which we use the assumption of axisymmetry is to allow us to apply our inferred velocity-radius relation Eq. (40) to the entire wind (i.e., to every azimuth). Without the assumption of an axisymmetric wind velocity we can only say that along this particular streamline velocity varies with radius according to Eq. (40), but on other streamlines the velocity-radius relation may be different: for streamlines originating from the surface at azimuth $\phi_{0}$ we have a velocity-radius relation $w_{\mathrm{s}}\left(x, \phi_{0}\right)$. Were we to observe several discrete narrow DACs simultaneously we could use the inversion procedure of this section to infer $w_{\mathrm{s}}\left(x, \phi_{0}\right)$ for each of them (i.e., for each $\phi_{0}$ ), potentially recovering a non-axisymmetric wind velocity law. We discuss the implications of this in relation to wide-stream DAC inversions in Sect. 4.2.

\subsubsection{Narrow stream inversion using the continuity equation}

Can we extract more information from narrow-stream DAC observations by making use of the optical depth of the absorption feature and how it varies with phase, possibly allowing $\Omega(r)$ to be inferred rather than assumed? It turns out that we can, but at a cost. Whereas, as we have just discussed, the narrowstream inversion procedure of Sect. 3.4.2 requires no knowledge of streamlines in the vicinity of the CDR stream, to interpret optical depth information requires the continuity equation and therefore knowledge of the variation of the wind velocity around the CDR stream, since the continuity equation relates the divergence of neighbouring streamlines to the change in density along them, and thus, through Eq. (1) to the change in optical depth of the corresponding DAC. It follows, therefore, that we must employ our model assumption on the axisymmetry of the velocity law (or some alternative) to take advantage of narrow-stream optical depth variations.

If we consider a narrow DAC generated by a $\delta$-function surface density function $F_{0}\left(\phi_{0}\right)=A \delta\left(\phi_{0}\right)$, then from Eq. (7) the dimensionless optical depth function becomes

$f(w, \phi)=A \mathcal{P}(w) \delta(\phi-\Delta \phi(w))$,

and we can think of the DAC either as a $\delta$-function in velocity at any time $\phi$, or as a $\delta$-function in phase at any velocity.

The inversion procedure of Sect. 3.4.2 was based on the observed DAC velocity as a function of phase $w_{\mathrm{p}}(\phi)$, which is precisely the inverse function of $\Delta \phi(w)$ in Eq. (44). Can we use optical depth measurements to determine instead the $\mathcal{P}(w)$ function in Eq. (44), and then use this to further constrain the parameters of the wind?

As we mentioned in Sect. $2, \mathcal{P}(w)$ represents the line profile of the absorption component of the wind in the absence of DACs, and we show in Sect. 4.3 that it can be used to determine the wind law $w_{\mathrm{s}}(x)$ without knowledge of $\Omega(r)$

To determine $\mathcal{P}(w)$ from observations, given the dynamical spectrum from Eq. (44), we must integrate over the spectrum to obtain the amplitude $A$ of the $\delta$-function DAC feature. As we discuss in Sect. 4.2.2, it is advantageous to think of the variation of optical depth with phase at fixed $w$, rather than in terms of the spectrum at fixed phase. This is seen clearly here if we integrate over $f(w, \phi)$ in Eq. (44) to obtain $\mathcal{P}(w)$. Integrating over $\phi$ gives

$\mathcal{A}(w)=\int_{0}^{2 \pi} A \mathcal{P}(w) \delta(\phi-\Delta \phi(w)) \mathrm{d} \phi=A \mathcal{P}(w)$,

which is what we want, whereas integrating over $w$ gives

$$
\begin{aligned}
\mathcal{A}(\phi) & =\int A \mathcal{P}(w) \delta(\phi-\Delta \phi(w)) \mathrm{d} w \\
& =A \int \mathcal{P}(w) \delta(\phi-\Delta) \frac{\mathrm{d} w}{\mathrm{~d} \Delta} \mathrm{d} \Delta \\
& =A \mathcal{P}(w) \frac{\mathrm{d} w}{\mathrm{~d} \Delta \phi}
\end{aligned}
$$

So from the DAC amplitude of Eq. (45) we can infer the velocity law of the wind as in Sect. 4.3, and can compare this with 
the law inferred in Sect. 3.4.2 to examine the consistency of our choice of rotation law, and ultimately to infer $\Omega(r)$. We will not pursue this further here, since it is just a limiting case of the wide-stream DAC inversion that we present in Sect. 4.3.

\section{Wide streams and the general DAC inversion problem}

\subsection{Acceleration of DAC peak $\tau$ from a wide stream}

In Sect. 3.1 we discussed the apparent Doppler acceleration of the narrow DAC feature arising from a stream which is narrow in $\phi_{0}$ (and therefore in $w$ ). In reality streams do have substantial widths, as evidenced by the finite Doppler width of DACs and the fact (Kaper et al. 1999) that they must have sufficient spatial extent to cover a large enough fraction of the stellar disk for DAC absorption to be important. Thus narrow stream analysis must be treated with caution, as indeed must analyses (e.g., Kaper et al. 1999) that make restrictive parametric assumptions (e.g., Gaussian) on the shape of the profile of either the DAC $f(w)$ or of the stream density $F_{0}\left(\phi_{0}\right)$. For wide streams there is no unique $w(\phi)$ but rather a profile $\tau(w, \phi)$ which depends (Eq. (7)) not only on $w_{\mathrm{s}}(x)$ but also on $W(x)$ and $F_{0}\left(\phi_{0}\right)$. For these one has to discuss the acceleration of a feature (or of the mean over some $w$ interval) - for example of the value $w=w_{*}(\phi)$ of the Doppler speed at which $\tau(w, \phi)$ maximises. In general the apparent (pattern) acceleration $a_{*}$ of $w_{*}(\phi)$ may depend on the mass loss flux profile function $F_{0}\left(\phi_{0}\right)$ as well as on $w_{\mathrm{s}}$ and $W$. Here we examine the acceleration of $w_{*}(\phi)$ for general $F_{0}\left(\phi_{0}\right), w_{\mathrm{s}}(x)$ and $W(x)$ to see how much $F_{0}\left(\phi_{0}\right)$ affects our earlier narrow stream result that the apparent DAC pattern acceleration from a narrow stream exceeds that from an absorbing puff moving radially with the same matter speed.

We will denote by $a_{*}=\mathrm{d} w_{*} / \mathrm{d} \phi$ the dimensionless Doppler "acceleration" in the "time" coordinate $\phi$ of the DAC peak. For a very narrow stream where there is a unique Doppler speed this will just be the pattern acceleration $a_{\mathrm{p}}$ we derived earlier, viz.

$a_{\mathrm{p}}=\frac{1}{\mathrm{~d} \Delta \phi / \mathrm{d} w_{\mathrm{p}}}=\frac{1}{\Delta \phi^{\prime}\left(w_{\mathrm{p}}\right)}$.

For a broad stream $w=w_{*}$ is where $f(w, \phi)$ from Eq. (7) maximises in $w$-i.e., $\partial f / \partial w=0$ which can be written

$$
\begin{aligned}
& \frac{\mathrm{d} \log \mathcal{P}}{\mathrm{d} w}\left(w=w_{*}\right)= \\
& \frac{\mathrm{d} \log F_{0}}{\mathrm{~d} \phi_{0}}\left(\phi_{0}=\phi-\Delta \phi\left(w=w_{*}\right)\right) \frac{\mathrm{d} \Delta \phi}{\mathrm{d} w}\left(w=w_{*}\right) .
\end{aligned}
$$

Defining

$H(w)=\frac{\mathrm{d} \log \mathcal{P} / \mathrm{d} w}{\mathrm{~d} \Delta \phi / \mathrm{d} w} ; \quad L\left(\phi_{0}\right)=\mathrm{d} \log F_{0} / \mathrm{d} \phi_{0} ;$

$w_{*}(\phi)$ is given by

$H\left(w_{*}\right)=L\left(\phi-\Delta \phi\left(w_{*}\right)\right)$.

Differentiating Eq. (50) for $w_{*}$ with respect to $\phi$ we then get

$H^{\prime}\left(w_{*}\right) a_{*}=$

$\frac{\mathrm{d} L}{\mathrm{~d} \phi_{0}}\left(\phi_{0}=\phi-\Delta \phi\left(w_{*}\right)\right)\left[1-\Delta \phi^{\prime}\left(w_{*}\right) a_{*}\left(w_{*}\right)\right]$ where' denotes $\mathrm{d} / \mathrm{d} w$. Solving for $a_{*}$ we get for the "acceleration" of the DAC peak

$a_{*}\left(w_{*}\right)=\left[\Delta \phi^{\prime}\left(w_{*}\right)+\frac{H^{\prime}\left(w_{*}\right)}{\frac{\mathrm{d} L}{\mathrm{~d} \phi_{0}}\left(\phi\left(w_{*}\right)-\Delta \phi\left(w_{*}\right)\right)}\right]^{-1}$,

where $\phi\left(w_{*}\right)$ is given by Eq. (48). Finally we note from Eq. (47) that $\Delta \phi^{\prime}(w)=1 / a_{\mathrm{p}}(w)$ where $a_{\mathrm{p}}$ is the apparent (pattern) "acceleration" for a narrow stream at Doppler speed $w$. We can finally compare the wide stream DAC peak optical depth acceleration with the narrow-stream acceleration at the same $w$ as

$\frac{a_{*}}{a_{\mathrm{p}}}=\left\{1+\left[\frac{H^{\prime}(w) / \Delta \phi^{\prime}(w)}{L^{\prime}(\phi(w)-\Delta \phi(w))}\right]\right\}^{-1}=\frac{1}{1+\psi(w)}$,

where

$\psi(w)=\left[\frac{H^{\prime}(w) / \Delta \phi^{\prime}(w)}{L^{\prime}(\phi(w)-\Delta \phi(w))}\right]_{w=w_{*}}$.

The first property we note about this expression is that if $\mathcal{P}(w)$ is constant then $H^{\prime}(w)=0$ and $a_{\mathrm{p}}=a_{*}$ for all $w-$ i.e., the maximum DAC absorption peak in $\tau$ moves in exactly the same way as that from a narrow stream (and so faster than a puff i.e., $>a_{\mathrm{s}}$ ) regardless of the form of the mass loss flux distribution $F_{0}\left(\phi_{0}\right) . \mathcal{P}(w)=$ constant corresponds to $x^{2} w_{\mathrm{s}} w_{\mathrm{s}}^{\prime}=$ constant or $w(x)=(1-1 / x)^{1 / 2}-$ i.e., a $\beta$-law with $\beta=1 / 2$. This property can also be seen directly from Eq. (49) where constant $\mathcal{P}$ implies $H=0$ so that the equation for $w_{*}(\phi)$ is just $L\left(\phi-\Delta \phi\left(w_{*}\right)\right)=0$ or $\Delta \phi=\phi_{0 \text { peak }}$ where $\phi_{0 \text { peak }}$ is where the density function $F_{0}\left(\phi_{0}\right)$ peaks. That is, for constant $\mathcal{P}(w)$ $(\beta=1 / 2)$, the shape $f(w, \phi)$ of the moving DAC profile simply tracks the shape of $F_{0}\left(\phi_{0}\right)$ with a phase that changes with time according to the $\Delta \phi(w)$ law. We noted in Sect. 2 and will see again below (Sect. 4.2.2) that a generalised form of this result actually applies to all $w(x)-$ i.e. to general $\mathcal{P}(w)-$ in that rescaling the data $f(w, \phi)$ to $f(w, \phi) / \mathcal{P}(w)$ yields a DAC profile function which tracks the form of $F_{0}\left(\phi_{0}\right)$ with a phase shift $\Delta \phi(w)$. This property, which arises from the fact that $w, \phi$ only arise in a separable combination in the argument of the factor $F_{0}$ in expression (8) is what makes it possible to recover all three functions $w_{\mathrm{s}}(x), W(x)$ and $F_{0}\left(\phi_{0}\right)$ from $f(w, \phi)$

For the case $\mathcal{P} \neq$ constant (anything other than a $\beta=1 / 2$ velocity law), whether $a_{*}>a_{\mathrm{p}}$ or $a_{*}<a_{\mathrm{p}}$, i.e., whether the peak of a DAC from a wide stream accelerates faster or slower than that from a narrow stream, depends on whether $\psi<0$ or $\psi>0$ in Eq. (53).

We see that, in general, $\psi$ depends on $F_{0}\left(\phi_{0}\right)$ as well as on $w_{\mathrm{s}}(x), W(x)$, so that the acceleration $a_{*}$ of a spectral peak in the DAC optical depth $f$ from a broad stream is not the same as that $\left(a_{\mathrm{p}}\right)$ from a narrow one. This is because the shape of $F_{0}$ causes the spectral shape of $f$, including the behavior of its peak, to change with time. Now in Eq. (54) $\Delta \phi^{\prime}(w)=1 / a_{\mathrm{p}}(w)$ is always $>0$ while the sign of $L^{\prime}$ describes the concavity of $\log F_{0}$. Noting that

$L^{\prime}=\frac{\mathrm{d}}{\mathrm{d} \phi_{0}}\left[\frac{1}{F_{0}} \frac{\mathrm{d} F_{0}}{\mathrm{~d} \phi_{0}}\right]=-\left[\frac{1}{F_{0}} \frac{\mathrm{d} F_{0}}{\mathrm{~d} \phi_{0}}\right]^{2}+\frac{1}{F_{0}} \frac{\mathrm{d}^{2} F_{0}}{\mathrm{~d} \phi_{0}^{2}}$ 
we also see that, since $F_{0}^{\prime \prime}<0$ anywhere in the neighbourhood of a peak in $F_{0}\left(\phi_{0}\right), L^{\prime}<0$ so the sign of $\psi$, and hence of $a_{\mathrm{p}}-a_{*}$, is opposite to the sign of $H^{\prime}$.

To proceed further we need to adopt definite forms for $F_{0}\left(\phi_{0}\right)$ and for $w_{\mathrm{s}}(x)$. Taking a $\beta$-law $w(x)$ as an example we have

$H(w)=-\frac{2}{S}(2 \beta-1) \frac{w\left(2-w^{\frac{1}{\beta}}\right)}{\left(1-w^{\frac{1}{\beta}}\right)^{2}}$,

while $\Delta \phi^{\prime}(w)$ is given by Eqs. (12) and (17). Thus we find

$$
\frac{H^{\prime}(w)}{\Delta \phi^{\prime}(w)}=-2(2 \beta-1) w^{2-2 / \beta}\left[\beta+\frac{w^{1 / \beta}\left(3-w^{1 / \beta}\right)}{\left(1-w^{1 / \beta}\right)\left(2-w^{1 / \beta}\right)}\right]
$$

the sign of which is simply fixed by the sign of $\beta-1 / 2$ and is always $<0$ for all $\beta>1 / 2$. By Eq. (54) this means that if $F_{0}\left(\phi_{0}\right)$ is concave down at the $\phi_{0}$ relevant to the peak at $w=w_{*}$ then $\psi>0$ and so $a_{*}<a_{\mathrm{p}}$ for all $\beta$-laws of $(\beta>1 / 2)-$ i.e., the wide stream peak $f$ accelerates slower than that of a narrow stream - because the observed speed of the peak $\tau$ is affected by the angular density profile of the wide stream as well as by the velocities $w(x), W(x)$, which solely govern the narrow stream case discussed above and by Hamann et al. (2001).

To see how large this effect is we consider for convenience the particular form of $F_{0}$

$F_{0}\left(\phi_{0}\right)=\left[\frac{A+\phi_{0} / 2 \pi}{(1+1 / A)^{\phi_{0} / 2 \pi}}\right]^{B}$.

This resembles a Maxwellian function, is continuous across $\phi_{0}=2 \pi$, and has an asymmetric peak at a $\phi_{0}$ value, and with a sharpness, which depend on constant dimensionless parameters $A$ and $B$. For this form it proves possible, using Eq. (55) to get an explicit analytic expression for $L^{\prime}$ at the point $\phi_{0}=\phi\left(w_{*}\right)-\Delta \phi\left(w_{*}\right)$ where $f$ peaks in $w$ i.e., at $w=w_{*}$ given by Eq. (50), namely

$L^{\prime}\left(\phi_{0}=\phi\left(w_{*}\right)-\Delta \phi\left(w_{*}\right)\right)=$

$$
-\frac{B}{4 \pi^{2}}\left[\frac{2 \pi}{B} H\left(w_{*}\right)-\log \left(1+\frac{1}{A}\right)\right]^{2} .
$$

Inserting this $L^{\prime}$ and $H^{\prime}\left(w_{*}\right) / \Delta \phi^{\prime}(w)$ from Eq. (57) in Eq. (53), we obtain an explicit expression for $a_{*} / a_{\mathrm{p}}$ as a function of $w$ for this form of $F_{0}\left(\phi_{0}\right)$ in terms of $\beta, A, B$. We confirmed that for $\beta=0.5, a_{*} / a_{\mathrm{p}}=1$ for any stream parameters $A, B$ and also found that for any quite narrow stream the value was very close to unity as expected.

Thus using the peak optical depth point $w_{*}$ as if it were the unique $w_{\mathrm{p}}$ for a narrow stream is a good approximation and so can be used to deduce $w_{\mathrm{s}}(x)$ from $w_{*}(\phi)$ as described in Sect. 3.4. For streams with $F_{0}$ of considerable width in $\phi_{0}$, corresponding to those DACs which have $f(w, \phi)$ rather broad in $w$ at small $w$ (cf. figures of data in Massa et al. 1995 and of simulations in Cranmer \& Owocki 1996) the results can be very different and quite complex since the evolution of $w_{*}(\phi)$ is strongly influenced by the stream density profile

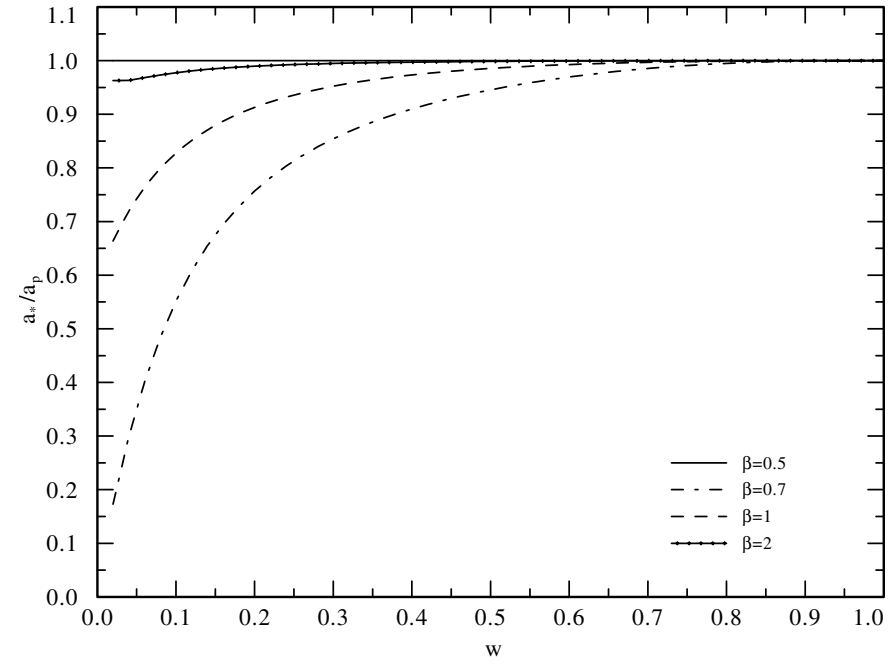

Fig. 5. Ratio of apparent accelerations of a wide stream DAC peak $\left(a_{*}(w)\right)$ to a narrow stream DAC $\left(a_{\mathrm{p}}(w)\right)$ for various $\beta$-laws for the input mass loss function with constants $A, B$ specified in the text

function $F_{0}\left(\phi_{0}\right)$. (Note that the DAC from a stream of any width in $\phi_{0}$ always becomes narrow in $w$ as $w \rightarrow 1$ since all the material eventually reaches terminal speed.) Results are shown for various $\beta$ values in Fig. 5 for $A=0.1, B=10$ which correspond to the fairly extreme case of a stream with a half width $F_{0}\left(\phi_{0}\right)$ of about 0.25 in $\phi$.

We see that in such cases the wide stream peak acceleration $a_{*}$ can be much less than the narrow stream result $a_{\mathrm{p}}$ especially for smaller values of $w$, and particularly for $\beta$ close to but greater than 0.5 which is thus a singular case. This means that, for DACs which are wide in $w$ at any stage in their development, estimating $w_{\mathrm{s}}(x)$ from, or even just fitting a value of $\beta$ to, data by applying narrow stream results to the acceleration of the DAC peak can be very misleading. The essential point here is that recurrent DAC data $f(w, \phi)$ contain much more information than on just $w_{\mathrm{s}}(x)$ but also on $F_{0}\left(\phi_{0}\right)$ and $W(x)$. To utilise this information content fully we have to treat the inverse problem, using both $w$ and $\phi$ distributions of $f(w, \phi)$. We show how this can be done in the next section.

\subsection{Inversion of wide-stream $f(w, \phi)$ for the wind characteristics}

In Sect. 3.4.2 we addressed the problem of recovering the $w(x)$ law from the observed $w_{\mathrm{p}}(\phi)$ pattern in the dynamic spectrum for a narrow DAC (representing matter flowing along a single streamline, from a single point on the stellar surface), and we showed that if we assume a rotation law (constant angular momentum, $\Omega(r) \sim 1 / r^{2}$, say) we can recover the spiral pattern $x_{\mathrm{s}}(\phi)$ and velocity law along that streamline without any consideration of neighbouring streamlines (i.e., without taking advantage of mass continuity or knowing $\mathrm{d} v / \mathrm{d} r$ ). Since a wide-stream DAC can be thought of as a collection of narrow streams from many (all) points on the stellar surface, surely we can apply the narrow-stream inversion procedure for each streamline, using the $w_{\mathrm{p}}\left(\phi, \phi_{0}\right)$ from each $\phi_{0}$ on the surface to 
obtain the spatial spiral law $x\left(\phi, \phi_{0}\right)$ for that streamline and the velocity-radius relation $w_{\mathrm{s}}\left(x, \phi_{0}\right)$ along each spiral, thus recovering the (in general non-axisymmetric) wind law $w_{\mathrm{s}}(x, \phi)$ without using our model assumption of an axisymmetric wind velocity (Sect. 2). In fact, we could do better even than this, because, if we obtain the velocity-radius relation along every streamline from the surface we have the wind velocity everywhere in the wind; we then know how it varies in the vicinity of every streamline and can calculate the derivatives necessary to apply the continuity equation and thus make use of the optical depth variations along streamlines as we discussed in Sect. 3.4.3. These variations would only be consistent with the observed $\tau(w, \phi)$ if the rotation law $\Omega(r)$ that we used to find the streamlines was correct, allowing us, in principle, to infer $\Omega(r)$ as well as the velocity law, thus giving all required wind parameters for a general non-axisymmetric wind.

Why don't we apply this procedure to the wide-stream DAC inversion problem? The answer is obvious: dynamic spectra do not come with the "velocity spirals" $w_{\mathrm{p}}\left(\phi, \phi_{0}\right)$ drawn on. It may be possible in general to draw many different spiral patterns on top of the dynamic spectrum of a wide DAC that give consistent inversions for $w(x, \phi)$, and it is certainly not obvious how, given just the dynamic spectrum, such a set of streamline spirals could be unambiguously chosen. As a result, the inference of a general azimuthally varying velocity law from recurrent DAC data is not a simple matter. We sidestep this issue here by introducing our model assumption of Sect. 2 (namely axial symmetry) to reduce the wide-stream inverse problem effectively to the narrow stream procedure (in a certain sense), but with the inclusion of mass continuity (Sect. 3.4.3). In fact, with our model assumption allowing us to make use of the continuity equation, wide-stream inference closely parallels the narrowstream problem with continuity of Sects. 3.4.2 and 3.4.3. As we will show, with this simple model assumption we are able to recover all characteristics of the wind velocity and CDR density.

\subsubsection{Forward problem}

The forward problem for wide-stream DACs involves the calculation of the dynamical spectrum $f(w, \phi)$ from $F_{0}\left(\phi_{0}\right), w_{\mathrm{s}}(x)$ and $W(x)$ :

1. From $w_{\mathrm{s}}(x)$ and $W(x)$ find the phase-shift function $\Delta \phi(w)$, which is just the inverse function of the "velocity spiral" $w_{\mathrm{p}}(\phi)$ found for the narrow-stream forward problem in Sect. 3.4.1;

2. Calculate $\mathcal{P}(w)$ from $w_{\mathrm{s}}(x)$ according to Eq. (6);

3. For each $w$ calculate $f(w, \phi)$ in Eq. (7) by phase-shifting $F_{0}\left(\phi_{0}\right)$ through $\Delta \phi(w)$ and multiplying by $\mathcal{P}(w)$.

\subsubsection{Inversion of $f(w, \phi)$ to find $w_{s}(x)$ for given $W(x)$}

In Sect. 3.4 we showed how the actual stream matter speed $w_{\mathrm{s}}(x)$ could be derived from the apparent DAC speed for a narrow stream. For a wide stream one might think of a similar method, using the apparent speed of DAC peak optical depth (i.e., the motion in $\phi$ of the $w=w_{*}$ at which $\partial f / \partial w=0$ ). However a better approach here is actually to consider rather the variation with $w$ of the time $\phi=\Phi(w)$ at which the optical depth at $w$ maximises, i.e., the $\phi(w)$ at which $\partial f / \partial \phi=0$. In fact, as a moment's thought shows, if we can identify any feature in the surface density profile $F_{0}\left(\phi_{0}\right)$, such at its peak at $\phi_{0 \text { peak }}$, say, and follow it as it flows out through the wind then we are precisely determining the velocity spiral for the single streamline emanating from the point $\phi_{0 \text { peak }}$. We can then apply the narrowstream inversion procedure of Sect. 3.4.2 to this spiral to infer the wind velocity law (strictly, to infer $w_{\mathrm{s}}\left(x, \phi_{0_{\text {peak }}}\right)$, but this is universal, i.e., independent of $\phi_{0}$, by our model assumption). Owing to the axisymmetry of the streamlines (Sect. 2) the way to trace the movement of the peak is to examine the variation of the optical depth with phase at each $w$, since, from Eq. (7), at fixed $w$ the variation of $f$ with $\phi$ is just proportional to $F_{0}$ phase-shifted by $\Delta \phi(w)$.

The position of the peak can be found from

$\frac{\partial f}{\partial \phi} \equiv L\left(\phi_{0}=\phi-\Delta \phi(w)\right)=0$

(cf. Eq. (7)). We know the peak occurs at $\phi_{0}=\phi_{0 \text { peak }}$, so the solution of (60) is simply

$\Phi(w)=\phi_{0 \text { peak }}+\Delta \phi(w)$

The result is thus independent of the form of $F_{0}$ except for the location of the peak, and depends only on the functions $w_{\mathrm{s}}(x)$ and $W(x)$ through $\mathcal{P}(w)$ and $\Delta \phi(w)$. The function $\Phi(w)$ in Eq. (61) is, apart from an irrelevant offset $\phi_{0 \text { peak }}$, just $\Delta \phi(w)$ the (inverse of) the velocity spiral function, which is the basis of the narrow-DAC inversion. We can now follow that procedure to infer $w_{\mathrm{s}}(x)$ given $\Omega(r)$ (i.e., $\left.W(x)\right)$ - see Eq. (40).

An alternative way to see explicitly how $w_{\mathrm{s}}(x)$ can be derived from Eq. (61) is to note that a monotonic function $Z(w)$ can be constructed from the data on $\Phi(w)$ and related to $W, w_{\mathrm{s}}$ by Eq. (72), viz.

$Z\left(w_{\mathrm{s}}\right)=\frac{\mathrm{d} \Delta \phi}{\mathrm{d} w}=S\left[\frac{1-W\left(x\left(w_{\mathrm{s}}\right)\right)}{w_{\mathrm{s}} w_{\mathrm{s}}^{\prime}}\right]_{w_{\mathrm{s}}=w}$.

For a known $W(x) \geq 0$, the right side is a known monotonic function of $x$ while the left side $Z$ is a monotonic function of $w_{\mathrm{s}}$ known from data. We can thus derive $w_{\mathrm{s}}(x)$ numerically. For the particular and commonly used case of $W=1 / x^{2}$ the expression reduces to the quadratic in $x$

$Z=S\left[x+\frac{1}{x}-2\right]$

with solution

$x\left(w_{\mathrm{s}}\right)=\left(1+\frac{Z\left(w_{\mathrm{s}}\right)}{2 S}\right)\left[1+\sqrt{1-\frac{1}{\left(1+Z\left(w_{\mathrm{s}}\right) / 2 S\right)^{2}}}\right]$.

\subsection{General inversion of $f(w, \phi)$ to find $w(x), W(x)$ and $F_{0}\left(\phi_{0}\right)$}

We now show that it is not necessary to know $W$ and that it is actually possible to recover all three functions $w(x), W(x)$ and $F_{0}\left(\phi_{0}\right)$ from data on $f(w, \phi)$, via the basic relationship

$f(w, \phi)=\mathcal{P}(w) F_{0}(\phi-\Delta \phi(w))$. 
This somewhat surprising result holds essentially because $F_{0}\left(\phi_{0}\right)$ is periodic and because $\phi$ and $w$ occur in the argument of $F_{0}$ only as a $w$-dependent phase shift combination with a scale factor $\mathcal{P}\left(w_{\mathrm{s}}\right)$ determining the basic data $f$ as already noted in Sect. 2.

We pointed out in Sect. 3.4.3 that use of quantitative optical depth information boils down ultimately to the determination of the "line profile function" $\mathcal{P}(w)$, which contains information about mass continuity contraints (Sect. 2). Here we show that $\mathcal{P}(w)$ may easily be found from the observed dynamic spectrum, and can be used to infer $w_{\mathrm{s}}(x)$ without considering the rotation law at all.

Denoting by $\bar{f}$ the mean value of $f$ at any fixed $w$ over any $2 \pi$-range of $\phi_{0}$ we have, from Eq. (7),

$\bar{f}(w)=\frac{1}{2 \pi} \int_{0}^{2 \pi} f(w, \phi) \mathrm{d} \phi=\bar{F}_{0} \mathcal{P}(w)$,

with

$\bar{F}_{0}=\frac{1}{2 \pi} \int_{0}^{2 \pi} F_{0}\left(\phi_{0}\right) \mathrm{d} \phi_{0}$,

so that within a factor $\bar{F}_{0}$ we have

$\mathcal{P}(w)=\frac{1}{x^{2} w w^{\prime}}=\frac{\bar{f}(w)}{\bar{F}_{0}}$.

We have now found $\mathcal{P}(w)$ directly from the optical depth data by averaging $f$ over $\phi$ at different $w$. We could instead have used the value of $f$ along a single indentifiable streamline (such as the peak streamline above, i.e., $f(w, \Phi(w))=$ $\left.\mathcal{P}(w) F_{0}\left(\phi_{0 \text { peak }}\right)\right)$, but averaging will generally reduce the effects of noise on the solution.

From Eq. (68) it follows that

$\int_{w}^{1} \bar{f}(w) w \mathrm{~d} w=\frac{\bar{F}_{0}}{x}$

Putting $x=1, w=0$ at the surface gives

$\bar{F}_{0}=\int_{0}^{1} \bar{f}(w) w \mathrm{~d} w$,

so we can determine the inverse solution for $x\left(w_{\mathrm{s}}\right)$ from $f(w, \phi)$ namely

$x\left(w_{\mathrm{s}}\right)=\frac{\int_{0}^{1} \bar{f}(w) w \mathrm{~d} w}{\int_{w_{\mathrm{s}}}^{1} \bar{f}(w) w \mathrm{~d} w}$,

from which, by inversion (since $x\left(w_{\mathrm{s}}\right)$ is monotonic) the matter velocity law $w_{\mathrm{s}}(x)$ can be recovered. Note that Eq. (71) only involves integrals of averages of the data funcion $f$ and we therefore expect the solution for $w_{\mathrm{s}}(x)$ to be very stable against data noise in $f$.

Next we show that we can use the combination of the streamline-based and $\mathcal{P}(w)$-based determinations of $w_{\mathrm{s}}$ (Eqs. (40) and (71)) to find the rotation law $\Omega(r)$. From Eq. (9) (see also Sect. 3.4.2) we can see that $\Delta \phi(w)$ depends on $W(x)$ and $w_{\mathrm{s}}(x)$. Since we have just determined $w_{\mathrm{s}}(x)$ from $\mathcal{P}(w)$ independently of $W(x)$ in Eq. (71) we can use this to determine $W(x)$. Differentiating $\Delta \phi$ with respect to $w$ gives

$$
\frac{\mathrm{d} \Delta \phi}{\mathrm{d} w}=\Delta \phi^{\prime}(w)=S\left[\frac{1-W(x(w))}{w_{\mathrm{s}} w_{\mathrm{s}}^{\prime}}\right]_{w_{\mathrm{s}}=w} .
$$

We know the left-hand side and $w_{\mathrm{s}}(x)$ from the data, and so we can rearrange Eq. (72) for $W(x)$ :

$W(x)=1-\frac{1}{S} w_{\mathrm{s}}(x) w_{\mathrm{s}}^{\prime}(x) \Delta \phi^{\prime}\left(w_{\mathrm{s}}(x)\right)$.

This solution for $W(x)$ involves a derivative of $\Delta \phi(w)$ and is therefore rather unstable to data noise unless subjected to smoothing procedures.

Finally, having found $\mathcal{P}(w)$ and $\Delta \phi(w)$ from the data we can, at each $w$, rescale the optical depth by $\mathcal{P}(w)$ and remove the phase shift $\Delta \phi(w)$ that results from the spiral shape to obtain the mass loss flux distribution function at the inner boundary, $F_{0}$, from $f(w, \phi)$ :

$F_{0}\left(\phi_{0}\right)=\frac{f\left(w, \phi_{0}+\Delta \phi(w)\right)}{\mathcal{P}(w)}$.

On the face of it, $F_{0}$ in Eq. (74) would depend on $w$, but, if the data truly conform to the form imposed by our kinematic corotating stream model then the result for $F_{0}\left(\phi_{0}\right)$ should be the same from any $w$ value used. In practice, a solution for $F_{0}\left(\phi_{0}\right)$ would be more sensibly and even more stably obtained from averaging Eq. (74) over $w$ (although if the noise on the dynamical spectrum varies strongly with $w$ it would be preferable to consider only those $w$ with the lowest noise). Note, however, that the solution for $F_{0}$ is directly proportional to the data $f$ with no derivatives involved so is very stable against data noise.

\section{Numerical inversion results for artificial data sets}

In Sect. 4.3 we have shown that, in principle, it is possible in the context of our kinematic wide-stream model to recover $w_{\mathrm{s}}(x)$, $F_{0}\left(\phi_{0}\right)$ and $W(x)$ from DAC optical depth data $f(w, \phi)$. We now investigate the extent to which it is actually possible to use this procedure to infer numerically the properties of a stellar wind from dynamical spectra, both in the case of perfect data and in the realistic case where there are errors on the observed dynamical spectrum.

To this end, we have tested the inversion procedure of Sect. 4.3 for a variety of artificial datasets, including $\beta$-laws with $\frac{1}{4} \leq \beta \leq 4$, velocity laws with a plateau (see below), rotation laws $W(x)=1 / x^{\gamma}$ with different $\gamma$, and various surface density profiles (wide and narrow Gaussians and sinusoidal modulations). In addition we have examined the effects on the inferred quantities of adding noise to the dynamical spectrum and also of "smearing" in velocity of the spectrum, simulating the influence of thermal and turbulent broadening intrinsic to the source.

Here we present the results of several representative inversions, choosing those that are most relevant to hot-star winds. Inversions for other wind parameters have comparable stability and accuracy. 
Table 1. The values of the plateau strength parameter $A$ in Eq. (76) for the wide and narrow plateaux for the three different underlying $\beta$-laws.

\begin{tabular}{|r||c|c|c|}
\hline \hline$\beta=$ & $\frac{1}{4}$ & $\frac{1}{2}$ & 1 \\
\hline \hline Wide plateau & 0.03 & 0.07 & 0.12 \\
\hline Narrow plateau & 0.004 & 0.007 & 0.010 \\
\hline
\end{tabular}

We concentrate on examining the dependence of DAC inversions on the characteristics of the underlying velocity law, and on the quality of the DAC data; the following rotation law and surface density profiles were used for all of the inversions shown in Figs. 6-18 ${ }^{1}$ :

- We take a constant angular momentum rotation law $W(x)=$ $1 / x^{2}$ (i.e., $\gamma=2$ in Eq. (11)).

- We adopt a Gaussian surface density profile

$$
F_{0}\left(\phi_{0}\right)=\frac{D}{\sqrt{2 \pi \sigma^{2}}} \mathrm{e}^{-\left(\phi_{0}-\mu\right)^{2} / 2 \sigma^{2}},
$$

with (dimensionless) amplitude $D=0.2$ and width $\sigma=$ 0.35 , centred on $\phi_{0}=\mu=0.3$. (Strictly speaking, we consider $F_{0}\left(\phi_{0}\right)$ given by folding Eq. (75) with period $2 \pi$, adding the contributions in each period together to ensure continuity across $\phi_{0}=2 \pi$.)

We are particularly interested in the effects of plateaux in the velocity law, such as those visible in the simulations of Cranmer \& Owocki (1996) and ascribed to the Abbott wave. The axial symmetry of the velocity field that we assume precludes the accurate modelling of Abbott-wave type effects (since the Abbott wave occurs in a spiral pattern, rather than at a fixed radius), but, as we would intuitively expect from the fact that the optical depth is inversely proportional to the velocity gradient (and as the inversion results in Figs. 6-14 show), a plateau in $w_{\mathrm{s}}(x)$ produces a very strong signal in the optical depth and can thus easily be recovered. Thus, the method recovers both density enhancements and velocity plateaux, if present. To illustrate this we consider three types of velocity law $w_{\mathrm{s}}(x)$ : pure $\beta$-laws, $\beta$-laws with a wide plateau (wide in radius) introduced around some radius, and $\beta$-laws with a narrow plateau. To introduce a plateau into a $\beta$-law $w_{\mathrm{s}}^{(\beta)}(x)$ (or any other velocity law) around some $x_{0}$ we can subtract a function $C(x)$ peaked around $x_{0}$ from $w_{\mathrm{s}}^{(\beta)}(x)$ so that the gradients of and $C(x)$ almost cancel at some point. Here we take

$C(x)= \begin{cases}A \cos ^{2} \frac{\pi\left(x-x_{0}\right)}{2 \delta}, & \text { for }\left|x-x_{0}\right|<\delta \\ 0, & \text { otherwise }\end{cases}$

where $\delta$ is simply the chosen width of the plateau region, and the amplitude $A$ controls the 'strength' of the plateau (how small the gradient becomes). Once appropriate values for $x_{0}$, $\delta$ and $A$ are chosen the plateau velocity law then becomes

$w_{\mathrm{s}}^{\text {plateau }}=w_{\mathrm{s}}^{(\beta)}(x)-C(x)$

(see the velocity-law panels of Figs. 6-8b, c). In all of the inversions shown we centre the plateau on $x_{0}=5$, and we consider

\footnotetext{
${ }^{1}$ Only the figures for $\beta=1$ are included in the printed version; the rest are available online as supplementary material.
}

two plateau widths, $\delta=3$ and $\delta=0.5$ (the values of $A$ used depend on the value of $\beta$ in the underlying velocity law, and are given in Table 1).

To summarise, three types of velocity laws are shown in the inversions of Figs. 6-14:

1. pure $\beta$-law $w_{\mathrm{s}}^{(\beta)}(x)($ Eq. (19));

2. $\beta$-law with a wide plateau $\left(x_{0}=5, \delta=3\right)$;

3. $\beta$-law with a narrow plateau $\left(x_{0}=5, \delta=0.5\right)$.

We present inversions for three underlying values of $\beta: \frac{1}{4}, \frac{1}{2}$ and 1 , chosen to bring out the effects we are examining most clearly. We show in Table 1 the values of the plateau constant $A$ for the wide and narrow plateaux shown in Figs. 6-14.

For the rotation law, surface density profile, and velocity laws set out above we calculated the resulting dynamical spectrum at $N_{v}=300$ uniformly-spaced velocity values between $v=0$ and $v=v_{\infty}$ and $N_{\mathrm{t}}=100$ times (i.e., rotational phases) throughout one rotation period by determining $\mathcal{P}(w)$ and $\Delta \phi(w)$ from Eqs. (6) and (9) respectively and then using Eq. (7) to obtain $f(w, \phi)$. (Although it is not necessary to use 300 velocity points to obtain acceptable resolution in general, the strong plateaux that we consider here give rise to extremely sharp features in the line profile, and failure to resolve these leads to significant truncation error in the calculation of the dynamical spectrum, and a consequent bias in the inversion.) These dynamical spectra were then inverted using the procedure described in Sect. 4.3 to infer $w_{\mathrm{s}}(x), W(x), F_{0}\left(\phi_{0}\right)$ (as well as $\Delta \phi(w)$ and $\mathcal{P}(w))$.

Figures 6, 7 and 8 show inversions for $\beta=\frac{1}{4}, \frac{1}{2}$ and 1 , respectively when no noise was added to the dynamical spectrum: the left panels show the dynamical spectra (data to be inverted), the right panels the inferred $w_{\mathrm{s}}(x), \Delta \phi(w) / 2 \pi, \mathcal{P}(w)$ and $F_{0}\left(\phi_{0}\right)$ (along with their "true" input values, which are virtually indistinguishable). Subfigures a), b) and c) are the inversions for each of the three velocity law types $(\beta$-law, wide plateau and narrow plateau, respectively.

Figures 9, 10 and 11 show inversions of exactly the same models as Figs. $6-8$, but with $10 \%$ noise added to the dynamical spectrum.

Finally, we illustrate in Fig. 14 an example of the effect of smearing the dynamical spectrum in velocity by convolving the error-free spectrum with a Gaussian blur with width $5 \%$ of the terminal velocity to artificially simulate thermal and turbulent broadening of the absorption from material at each radius. The inversions in Fig. 14 are for an underlying $\beta=1$ velocity law (results for each of the three velocity law types are shown, as usual). $10 \%$ errors are added to the smeared dynamical spectra. It is clear from the inversions that it is perfectly possible to infer the velocity law and surface density profile using the inversion procedure.

We have not yet discussed the rotation inference, however. We show in Figs. 15-17 the rotation law inferred for the noisefree inversions of Figs. 6-8. There are errors (mainly resulting from truncation error in the calculation of the model dynamical spectrum), but the quality of the inversion is good. Unfortunately, to calculate $W(x)$ requires differentiation of quantities derived from the data. This amplifies the errors significantly, and precludes accurate inference of $W(x)$ in the 
(a) Pure $\beta$-law
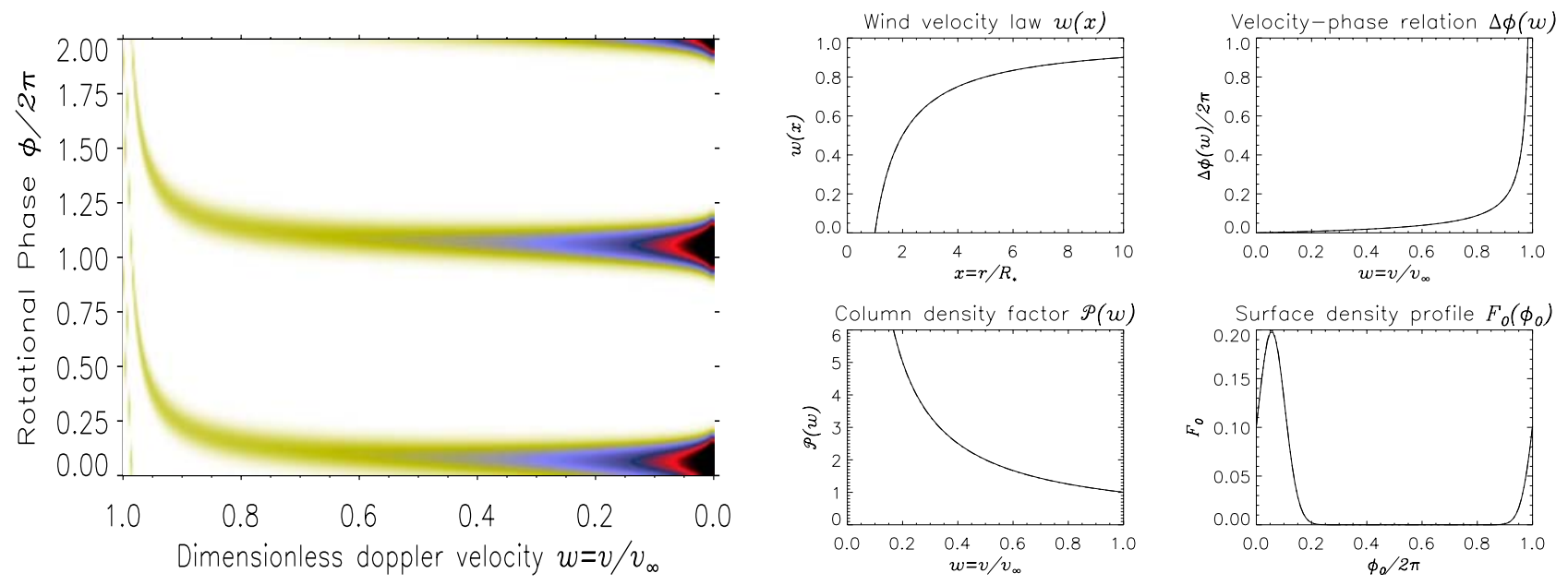

(b) Wide plateau
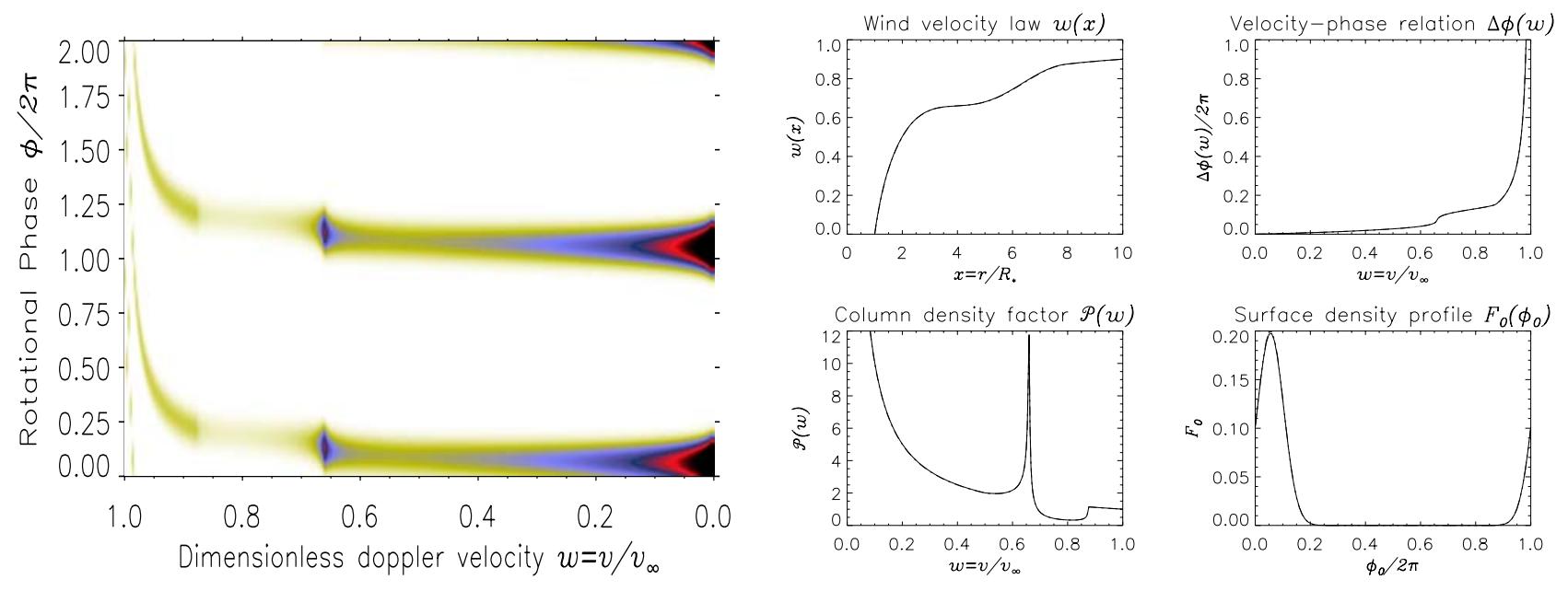

(c) Narrow plateau
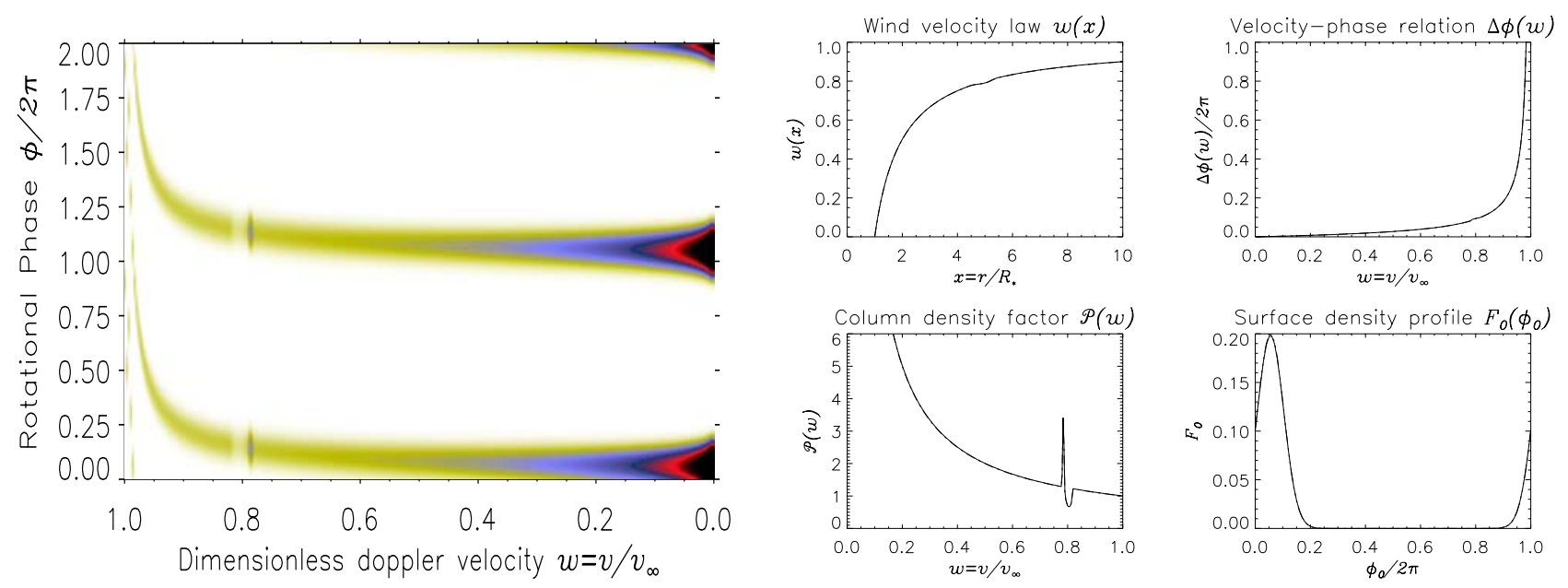

Fig. 8. $[\beta=1$, noise free] As in Fig. 6 , but for an underlying $\beta=1$ velocity law.

presence of errors as shown in Fig. 18. Looking at this more positively, though, it does indicate that the details of the wind's rotation velocity do not strongly influence the observed line profiles (physically this is because the angular velocity of fluid elements tends to zero quite quickly in general, so they follow the same path once they are beyond a few stellar radii), and 
(a) Pure $\beta$-law
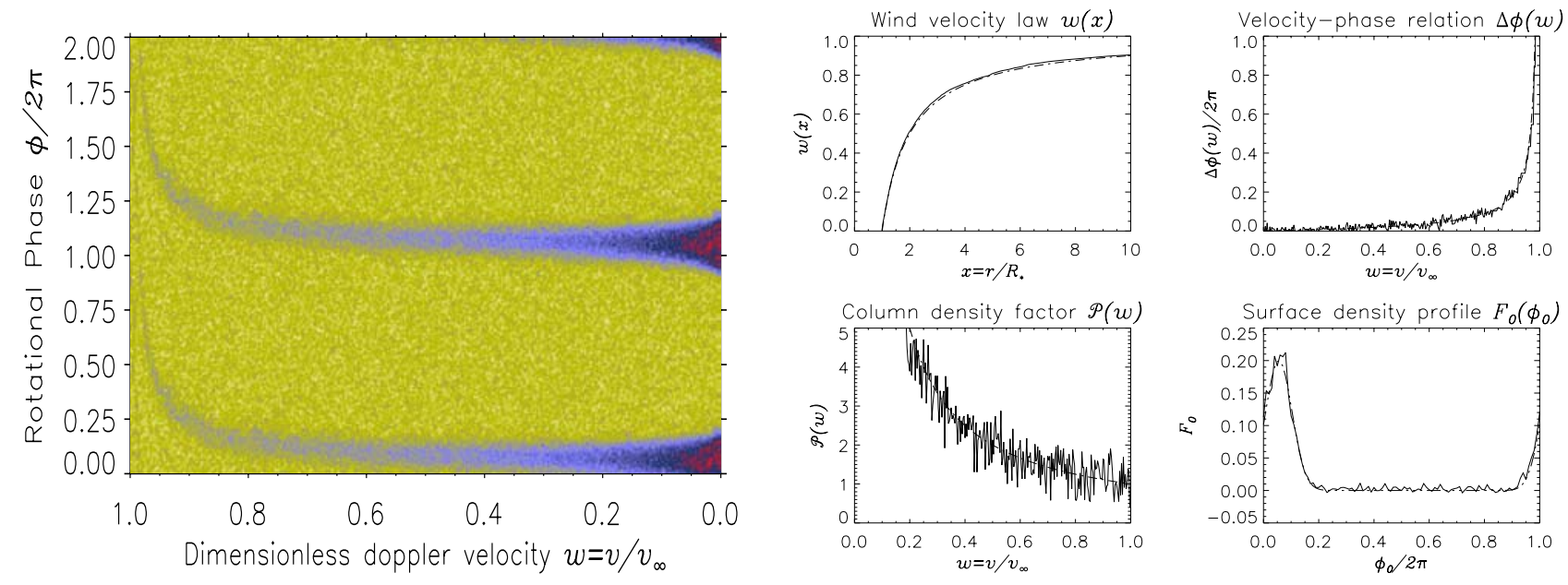

(b) Wide plateau
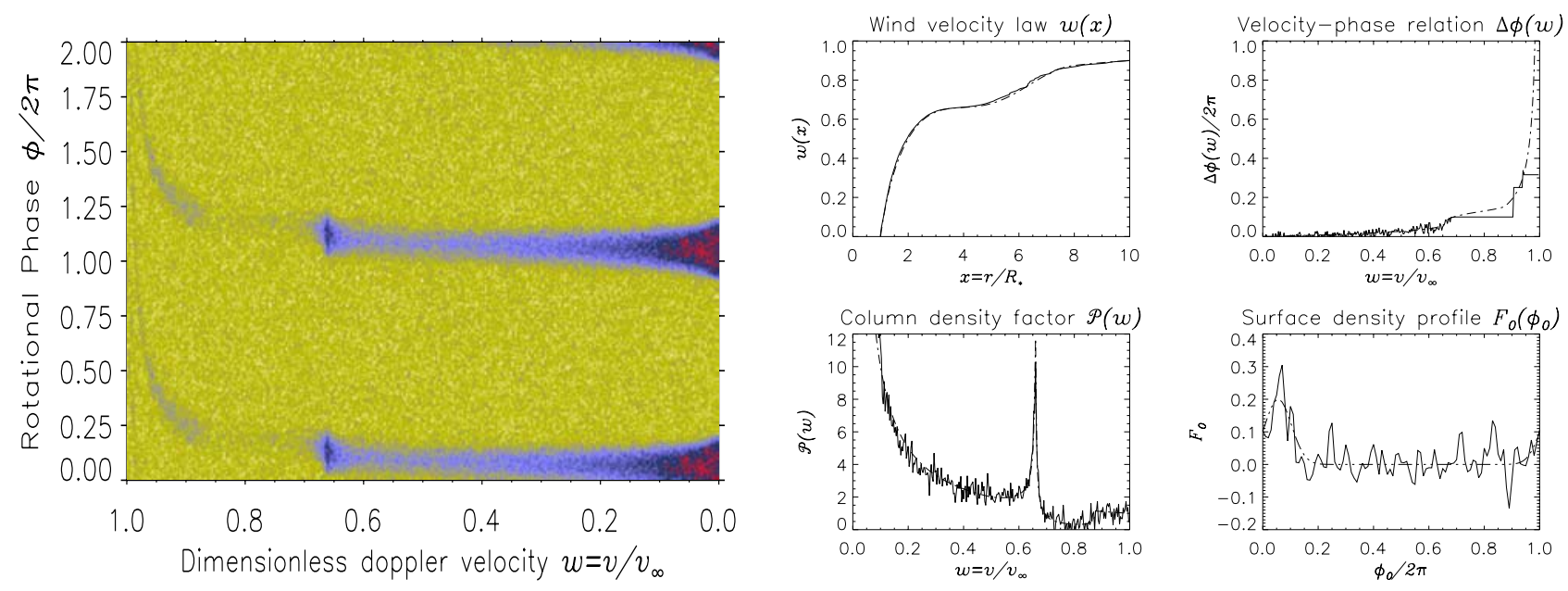

(c) Narrow plateau
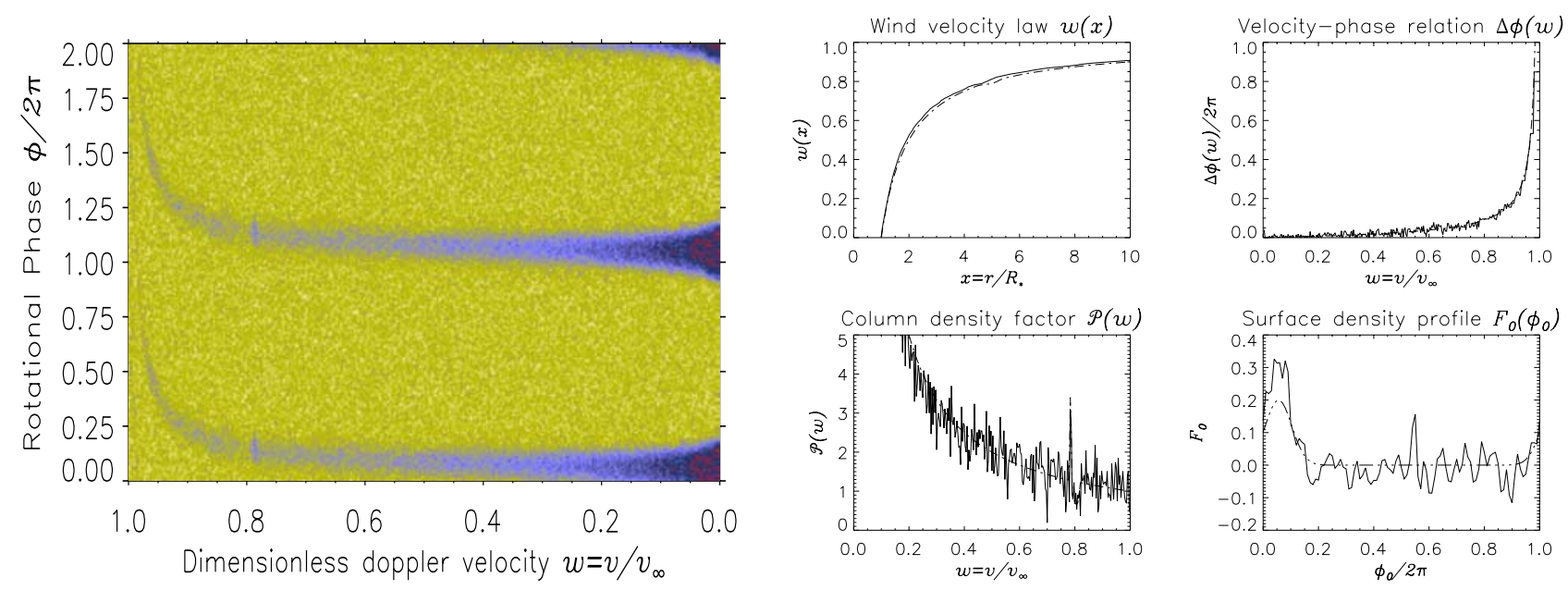

Fig. 11. $[\beta=1,10 \%$ errors $]$ As in Fig. 6 , but for an underlying $\beta=1$ velocity law and $10 \%$ errors.

uncertainty in $\Omega(r)$ does not prevent accurate inference of the other wind parameters. This means that it will not generally be neccessary to account accurately for the rotation law of the wind to make useful inferences about its structure.

\section{Discussion and application to real data}

The analysis above sheds much light on inferential wind stream diagnostics within the context of the kinematic model, and so 
(a) Pure $\beta$-law
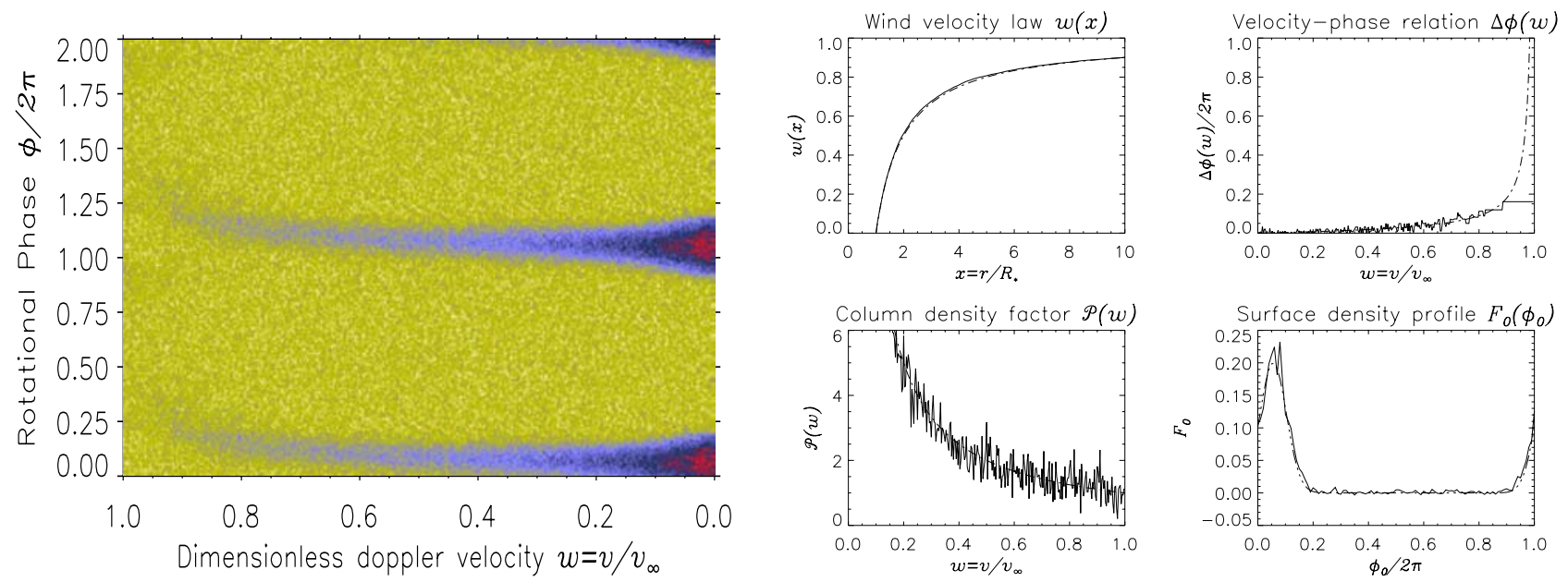

(b) Wide plateau
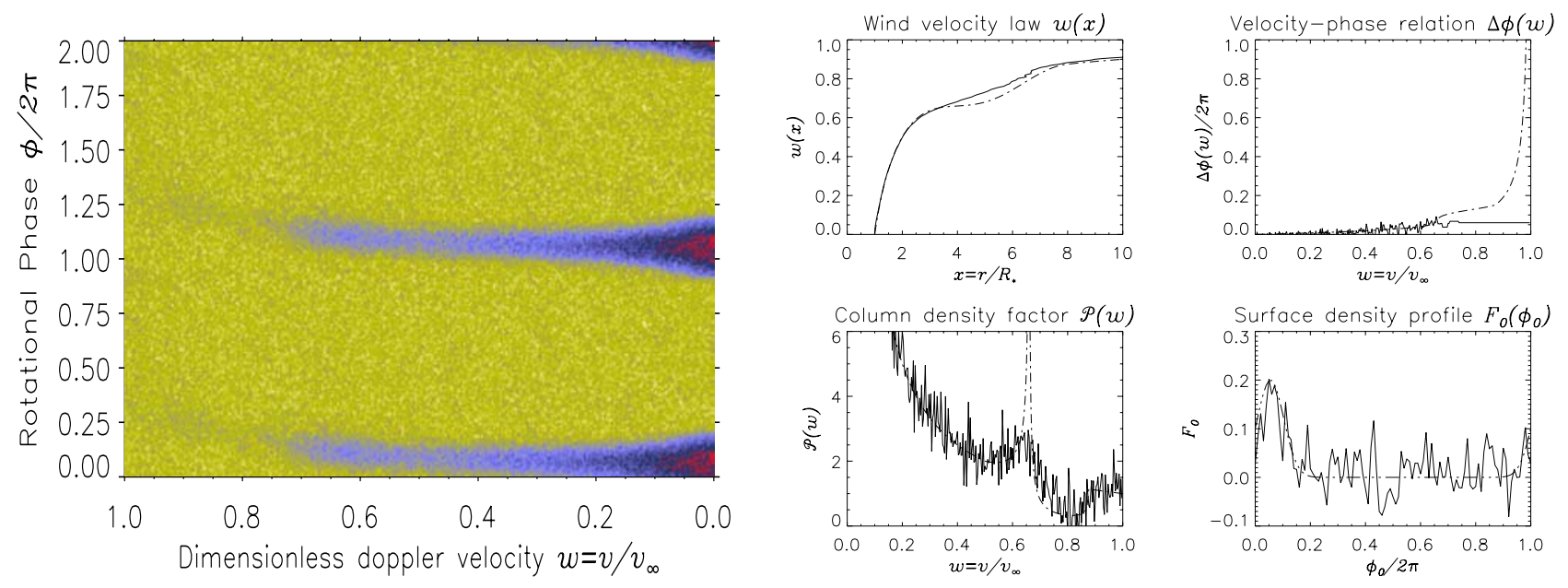

(c) Narrow plateau
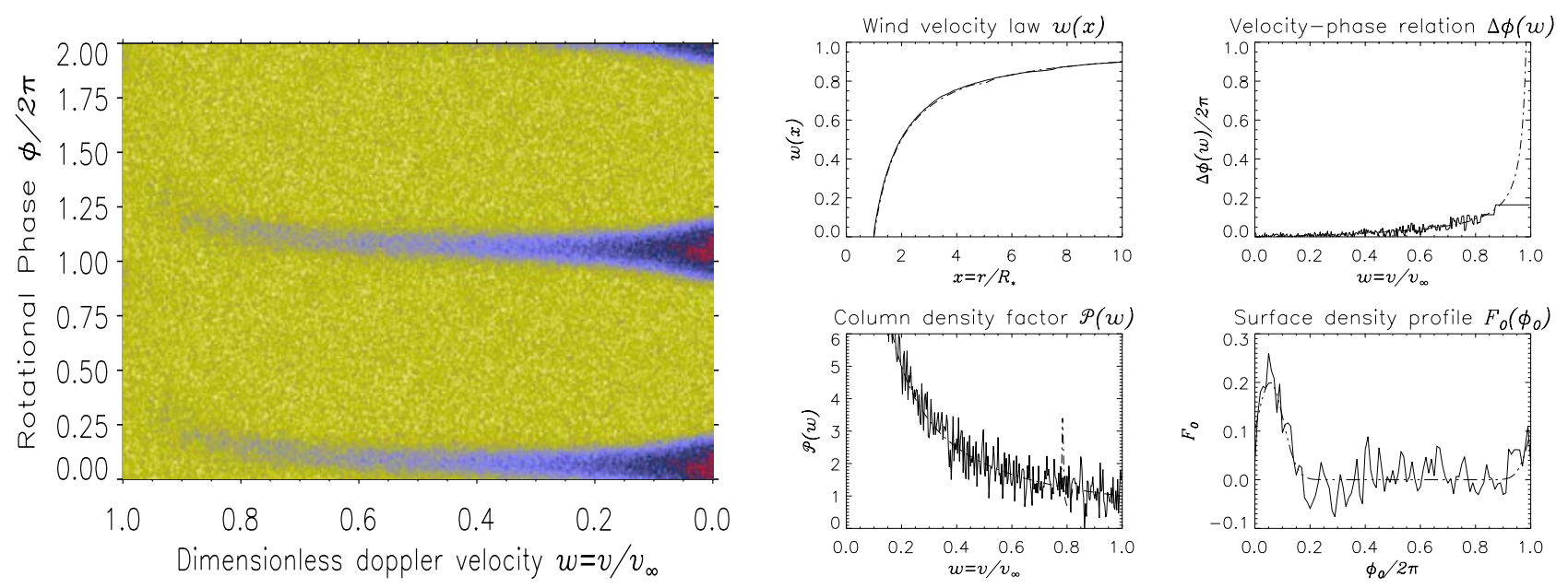

Fig. 14. [ $\beta=1,10 \%$ errors, $5 \%$ smearing] As in Fig. 6, but for an underlying $\beta=1$ velocity law, $10 \%$ errors and "velocity blurring" at $5 \%$ of the terminal velocity.

is a large step forward on the parametric forward fitting approach mainly used before in analysing data (Prinja \& Howarth 1988; Fullerton et al. 1997; Owocki et al. 1995) using the kinematic description. It is also a useful approach in bringing out the crucial importance of finite stream width and DAC feature profile in interpreting acceleration of features and in 
(a) Pure $\beta$-law

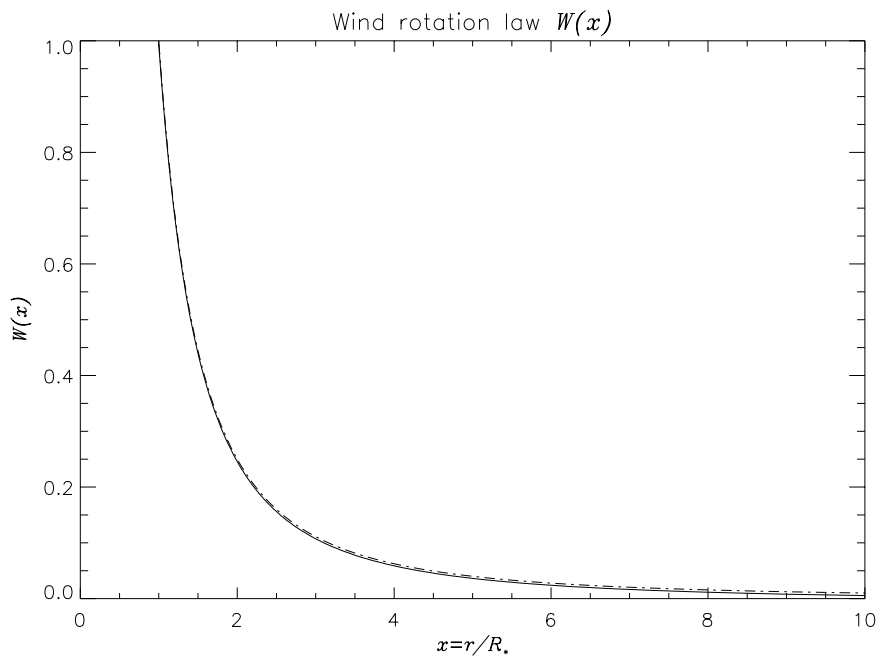

(b) Wide plateau

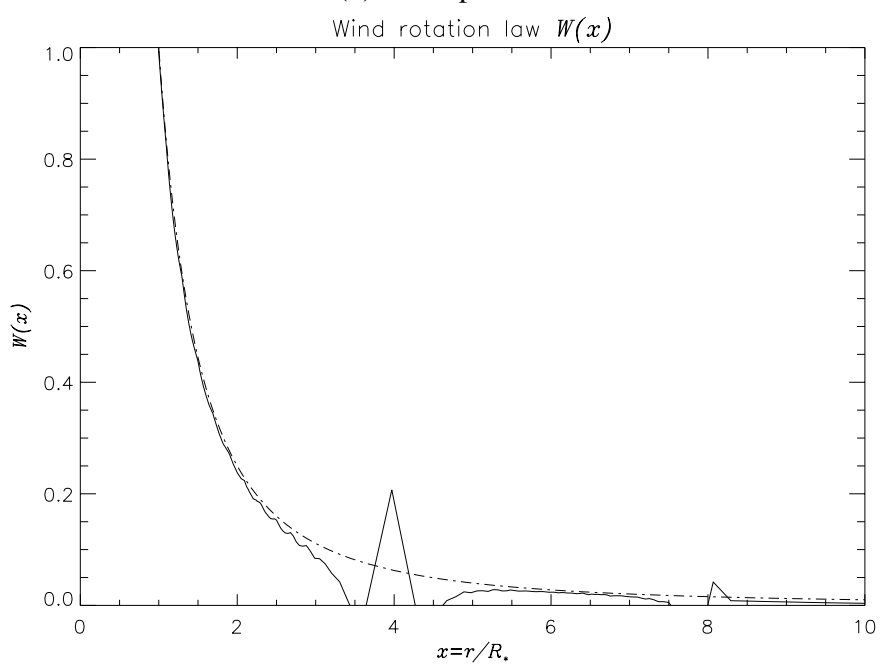

(c) Narrow plateau

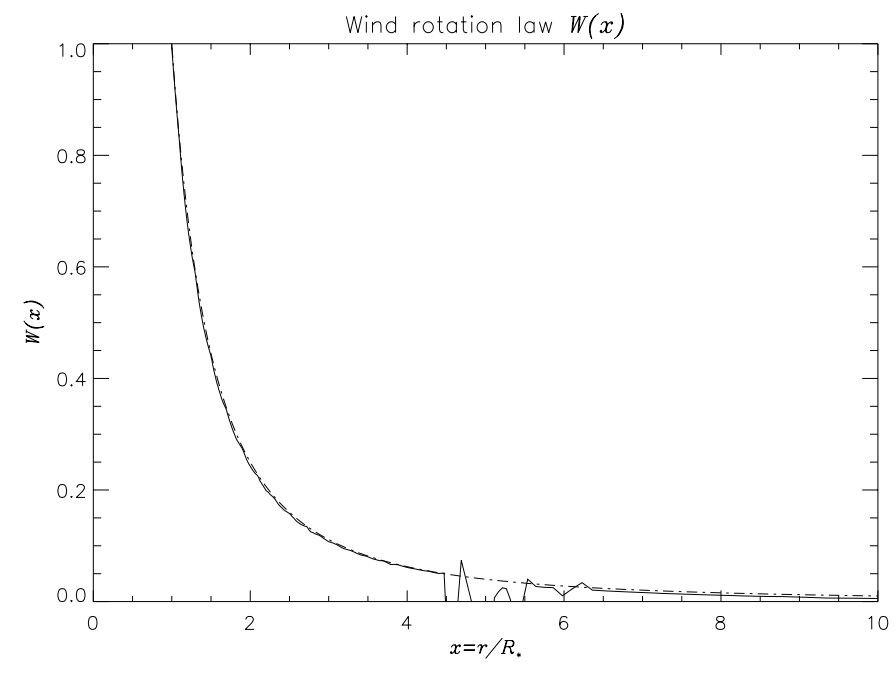

Fig. 17. $[\beta=1]$ As in Fig. 15, but for an underlying $\beta=1$ velocity law.

obtaining the fullest information from data on stream structure from DAC data which goes well beyond the velocity of

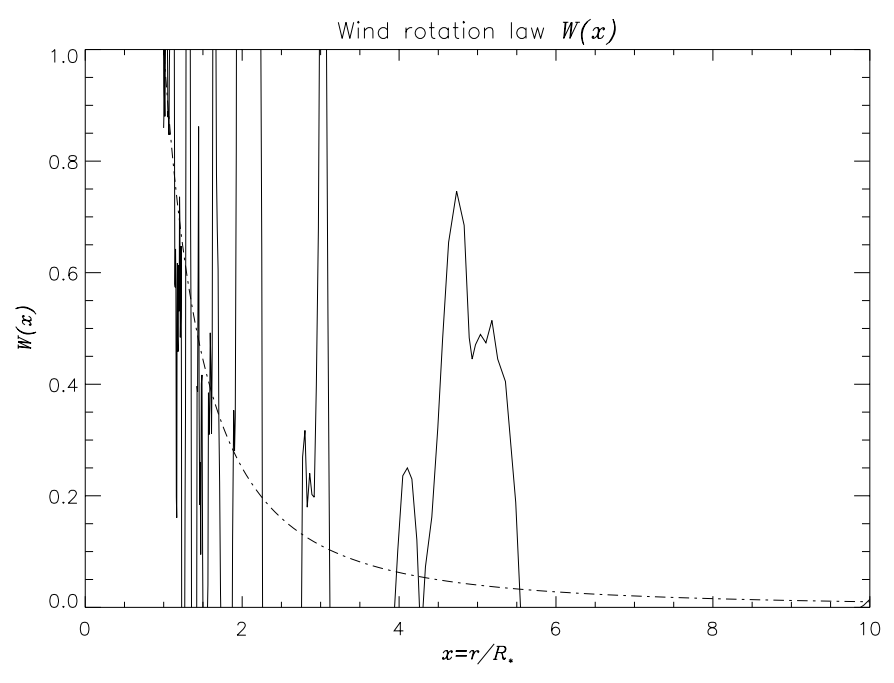

Fig. 18. Inferred wind rotation law, $W(x)$ for the dynamical spectrum shown in the left panel of Fig. 6a, that is, for a pure $\beta=1$ velocity law, with $10 \%$ errors added to the dynamical spectrum.

feature peaks discussed in Hamann et al. (2001). However, to take the model method forward it will be important to consider how well they match up to real data and to dynamical models (Cranmer \& Owocki 1996). These are the subjects of ongoing work and future papers (Krtička et al. 2003) but we consider them briefly here.

Recurrent hot star DAC features can be most clearly seen in UV resonance lines, such as SiIV and NV, though also present in $H_{\alpha}$ (de Jong 2000; de Jong et al. 2001), reflecting the presence of structure quite close to the stellar surface. There are a number of such datasets available from the IUE SWP instrument and we have made first attempts to apply our technique to the SiIV spectra of HD64760 (Massa et al. 1995). Results proved confusing mainly because the data contain distinct components (cf. Hamann et al. 2001). In particular there are strong slow DAC features lasting several rotation periods and crossing the weaker modulations at high $w$ corresponding to the CIR/CDRs which we have been discussing. Direct application of our solution to the full data set fails because the strong slow feature in no way conforms to the rotationally periodic character typical of the data features we set out to model. To progress, further work will be needed to try to isolate the two components and then apply our method to the rotationally periodic features only. As emphasised by Fullerton et al. (1997) exploration of data sets for the presence of several effects can be facilitated by phase binning all the data over a single period when effects not included in the model may show up as systematic residuals.

Another approach we are developing (Krtička et al. 2003) is to test the method against simulated data generated by dynamical models (Cranmer \& Owocki 1996) in which the dense wind streams are truly rotationally recurrent features. We do not expect to get wholly accurate recovery of $w_{\mathrm{s}}(x), F_{0}\left(\phi_{0}\right)$ since, as discussed earlier, the dynamical models, with the density feeding back on the veocity law though the radiative driver, produce structures in which $w_{\mathrm{s}}=w_{\mathrm{s}}(x, \phi)$ and not just $w_{\mathrm{s}}(x)$. Such features will not fully satisfy the assumption $w_{\mathrm{s}}=w_{\mathrm{s}}(x)$ of kinematic treatments used here and by others. The issue is to test 
how well the kinematically based inversion method we have developed enables approximate recovery of the density and velocity structure of the CIRs in the dynamical models. If reasonable first approximations to the forms $w_{\mathrm{s}}(x), F_{0}\left(\phi_{0}\right)$ of the dynamical models are recovered then we will be able to use the method on real data to provide first approximation inputs to dynamical simulations of the wind perturbation giving rise to the radiatively driven CIR. This will then enable dynamical modelling of real data sets to proceed faster than trial and error input of base perturbations to the dynamical code.

The other approximation which may limit the applicability of our method to real data is the "point-star" approximation. However, for a $\beta=1$ law speeds in the usefully observable range $0.5 \leq w \leq 0.9$ correspond to $2 \leq x \leq 10$ which should be reasonably approximated by the plane assumption, though again this can be tested by comparison of results with those of simulations incorporating curvature and finite star effects, to be undertaken in future work.

Acknowledgements. We wish to acknowledge the financial support of UK PPARC Research and Visitor Grants as well as University of Amsterdam Visitor funds. This paper has benefitted from numerous discussions with A.W. Fullerton, T. Hartquist, R. Prinja and J.P. Cassinelli. We are grateful for the comments of the referee, D. Massa.

\section{Appendix: The continuity equation}

The continuity equation was used in Sect. 2 to give Eq. (4). This can be proved in two ways - cf. Bjorkman (1992) and Bjorkman \& Cassinelli (1993).

1. Consider equatorial streamlines which are equally spaced in $\phi=\phi_{0}$ at the stellar surface. The streamline shape is given by

$\frac{1}{r} \frac{\mathrm{d} r}{\mathrm{~d} \phi}=\frac{v_{\mathrm{r}}}{v_{\phi}}$

which is independent of $\phi$ if $v_{\mathrm{r}}$ and $v_{\phi}$ are. Thus the streamlines are equally spaced in $\phi$ at any $r$. So the material flowing radially through an arc $R \delta \phi_{0}$ at the equator later flows radially through arc length $r \delta \phi=r \delta \phi_{0}$ at $r$ (but displaced in $\phi$ by amount $\Delta \phi=\phi-\phi_{0}$ due to the streamline curvature - Eq. (3)). If the surface density is $\Sigma(r)$ perpendicular to the equator then steady flow requires that

$\Sigma(r, \phi) r \delta \phi v_{\mathrm{r}}(r)=\Sigma_{o}\left(R, \phi_{0}\right) R \delta \phi_{0} v_{\mathrm{r}}(R)$,

so that

$\frac{\Sigma\left(r, \phi_{0}+\Delta \phi\right)}{\Sigma_{o}\left(R, \phi_{0}\right)}=\frac{v_{\mathrm{r}}(R) R}{v_{\mathrm{r}}(r) r}$.

But in 3-D flow near the equator $\Sigma \propto n(r) r$, so

$\frac{n\left(r, \phi_{0}+\Delta \phi\right)}{n_{0}\left(R, \phi_{0}\right)}=\frac{v_{\mathrm{r}}(R) R^{2}}{v_{\mathrm{r}}(r) r^{2}}$.

This simply means that the radial mass flow must be constant across all corresponding azimuthal elements.
2. More rigorously: the 3-D continuity equation at $\theta=0$ with $v_{\theta}=0$ is

$\frac{\partial}{\partial r}\left(n r^{2} v_{\mathrm{r}}\right)+r \frac{\partial}{\partial \phi}\left(n v_{\phi}\right)=0$.

Consider the variation $\delta X$ of the quantity $X=n r^{2} v_{\mathrm{r}}$

$\delta X=\delta r \frac{\partial X}{\partial r}+\delta \phi \frac{\partial X}{\partial \phi}$.

On a streamline this is, using (82),

$\delta X=\delta r\left[\frac{\partial X}{\partial r}+\frac{v_{\phi}}{r v_{\mathrm{r}}} \frac{\partial X}{\partial \phi}\right]$

and, again using the continuity Eq. (82), this is

$$
\begin{aligned}
\delta X & =\delta r\left[-r \frac{\partial}{\partial \phi}\left(n v_{\phi}\right)+\frac{v_{\phi}}{r v_{\mathrm{r}}} \frac{\partial\left(n r^{2} v_{\mathrm{r}}\right)}{\partial \phi}\right] \\
& =\delta r n r\left[-\frac{\partial v_{\phi}}{\partial \phi}+\frac{v_{\phi}}{r v_{\mathrm{r}}} \frac{\partial v_{\mathrm{r}}}{\partial \phi}\right],
\end{aligned}
$$

which is zero if $v_{\mathrm{r}}$ and $v_{\phi}$ are independent of $\phi$. Thus $X=n r^{2} v_{\mathrm{r}}$ is constant along streamlines. Equation (4) is just equivalent to $X(r)=X(R)$ on any streamline.

\section{References}

Abbott, D. C. 1980, ApJ, 242, 1183

Bjorkman, J. E. 1992, The effects of rotation on the winds from hot stars, Ph.D. Thesis, U. Wisconsin

Bjorkman, J. E., \& Cassinelli, J. P. 1993, ApJ, 409, 429

Brown, J. C., Richardson, L. L., Ignace, R., \& Cassinelli, J. P. 1997, A\&A, 325, 677

Craig, I. J. D., \& Brown, J. C. 1986, Inverse Problems in Astronomy (Adam Hilger Ltd)

Cranmer, S. R., \& Owocki, S. P. 1996, ApJ, 462, 469

de Jong, J. A. 2000, Ph.D. Thesis, University of Amsterdam

de Jong, J. A., Henrichs, H. F., Kaper, L., et al. 2001, A\&A, 368, 601

Feldmeier, A., \& Shlosman, I. 2002, ApJ, 564, 385

Fullerton, A. W., Massa, D. L., Prinja, R. K., Owocki, S. P., \& Cranmer, S. R. 1997, A\&A, 327, 699

Hamann, W.-R., Brown, J. C., Feldmeier, A., \& Oskinova, L. M. 2001, A\&A, 378, 946

Henrichs, H. F., Kaper, L., \& Nichols, J. S. 1994, A\&A, 285, 565

Kaper, L., Henrichs, H. F., Nichols, J. S., \& Telting, J. H. 1999, A\&A, 344,231

Kaper, L. 2000, in Thermal and Ionization Aspects of Flows from Hot Stars, ed. H. Lamers, \& A. Sapar, ASP Conf. Ser., 204, 3

Krtička, J., Barrett, R. K., Owocki, S., \& Brown, J. C. 2003, A\&A, submitted

Massa, D., Prinja, R. K., \& Fullerton, A. W. 1995, ApJ, 452, 842

Massa, D., Fullerton, A. W., Sonneborn, G., \& Hutchings, J. B. 2003, ApJ, 586, 996

Mullan, D. J. 1984, ApJ, 283, 303

Owocki, S. P., Cranmer, S. R., \& Fullerton, A. W. 1995, ApJ, 453, L37

Prinja, R. K., \& Howarth, I. D. 1988, MNRAS, 233, 123

Prinja, R. K., Massa, D., \& Fullerton, A. W. 2002, A\&A, 388, 587 


\section{Online Material}


J. C. Brown et al.: Inference of density stream properties from DACs, Online Material $p 2$

(a) Pure $\beta$-law
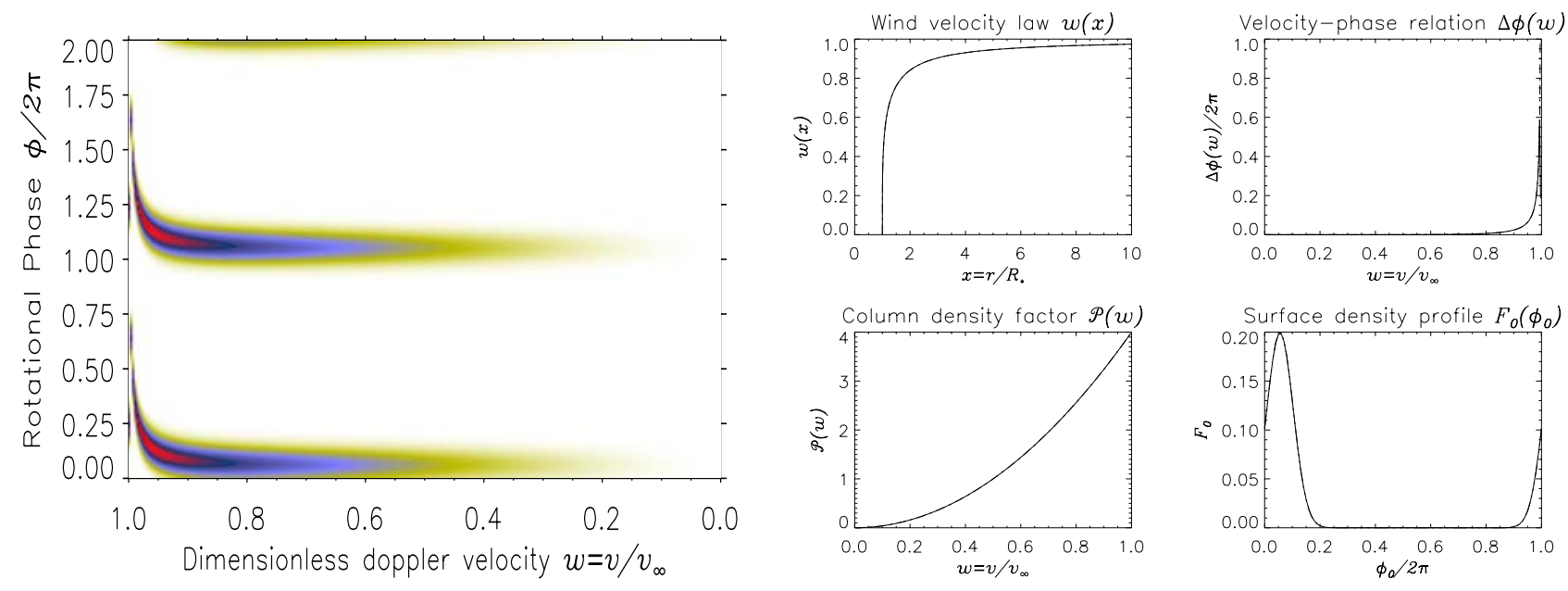

(b) Wide plateau
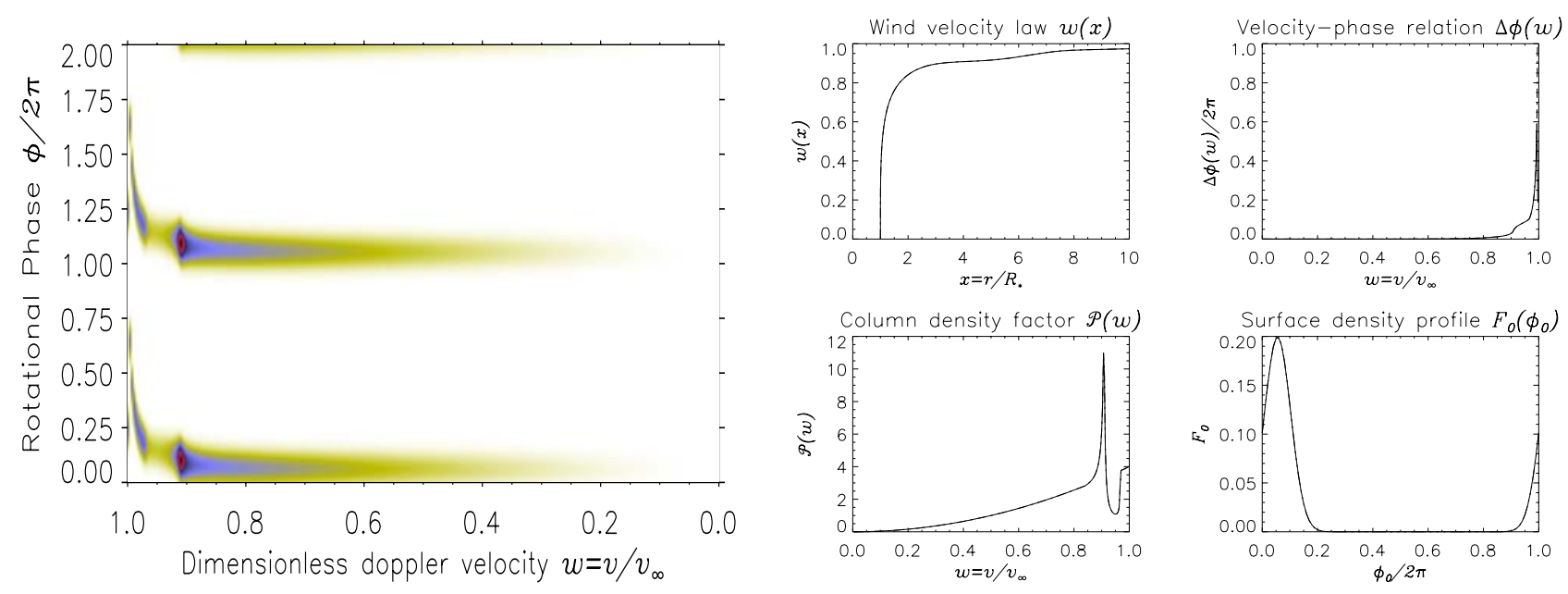

(c) Narrow plateau
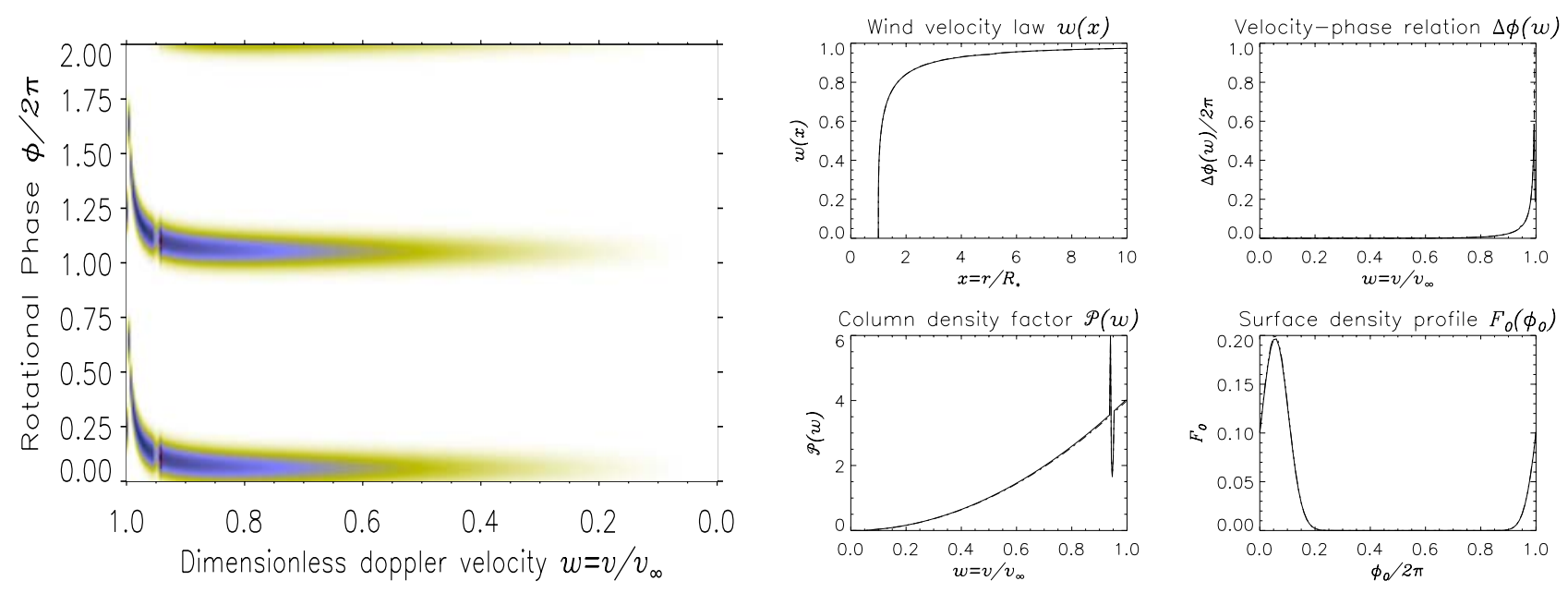

Fig. 6. $\left[\beta=\frac{1}{4}\right.$, noise free] Simulated dynamical spectra (left) and recovered wind profiles (right) for: a) pure $\beta$-law; b) $\beta$-law with a broad plateau (half-width $\Delta x=3$ ); $\mathbf{c}) \beta$-law with a narrow plateau $(\Delta x=0.5$ ). In b) and $\mathbf{c}$ ) the plateaux are centred on $x=5$ (see panels displaying the recovered $w(x)$ ). In all cases the underlying velocity law is a $\beta=\frac{1}{4}$ law, with a constant angular momentum rotation law and a broad Gaussian surface density profile (see the $F_{0}\left(\phi_{0}\right)$ panel). For the dynamical spectra the horizontal axis is doppler velocity, between $v=0$ (stellar surface) at the right of each panel and terminal velocity at the left. The vertical axis is time (i.e., rotational phase). No noise or blurring is added. In the right panels the "true" profiles are shown as dashed lines. Note that, for no noise or blurring, the true and inferred functions are almost completely indistinguishable. 
J. C. Brown et al.: Inference of density stream properties from DACs, Online Material p 3

(a) Pure $\beta$-law
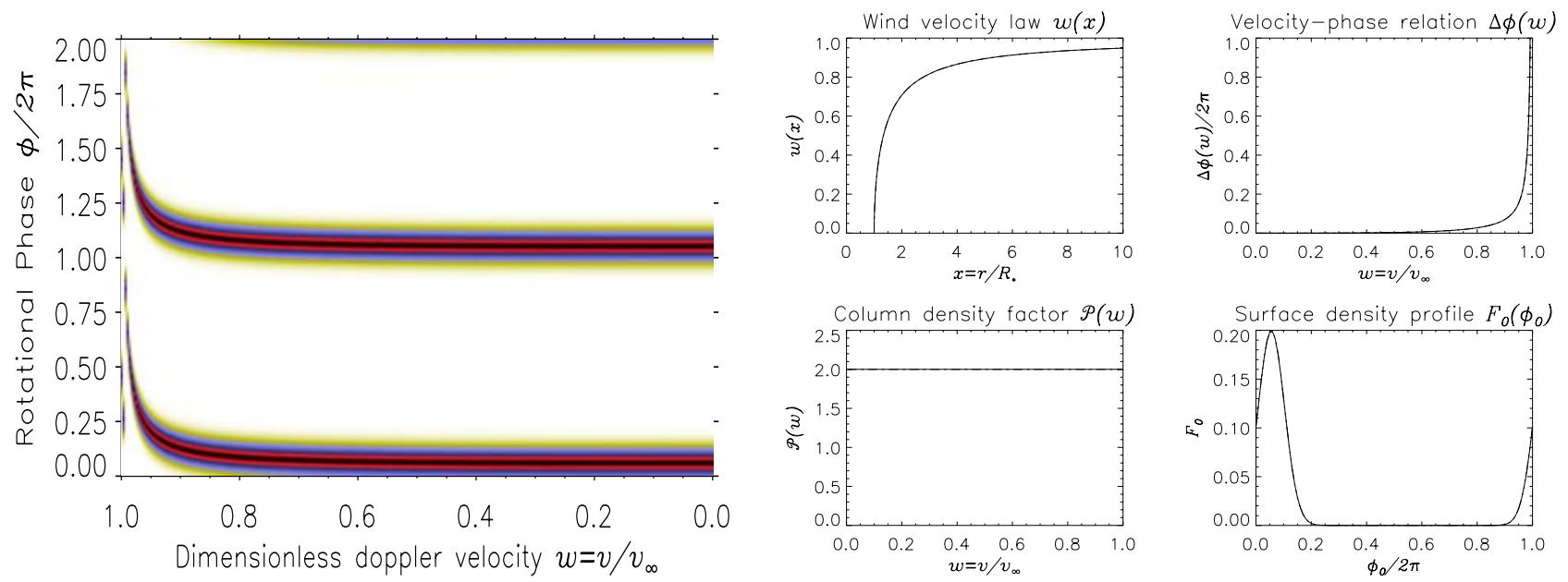

(b) Wide plateau
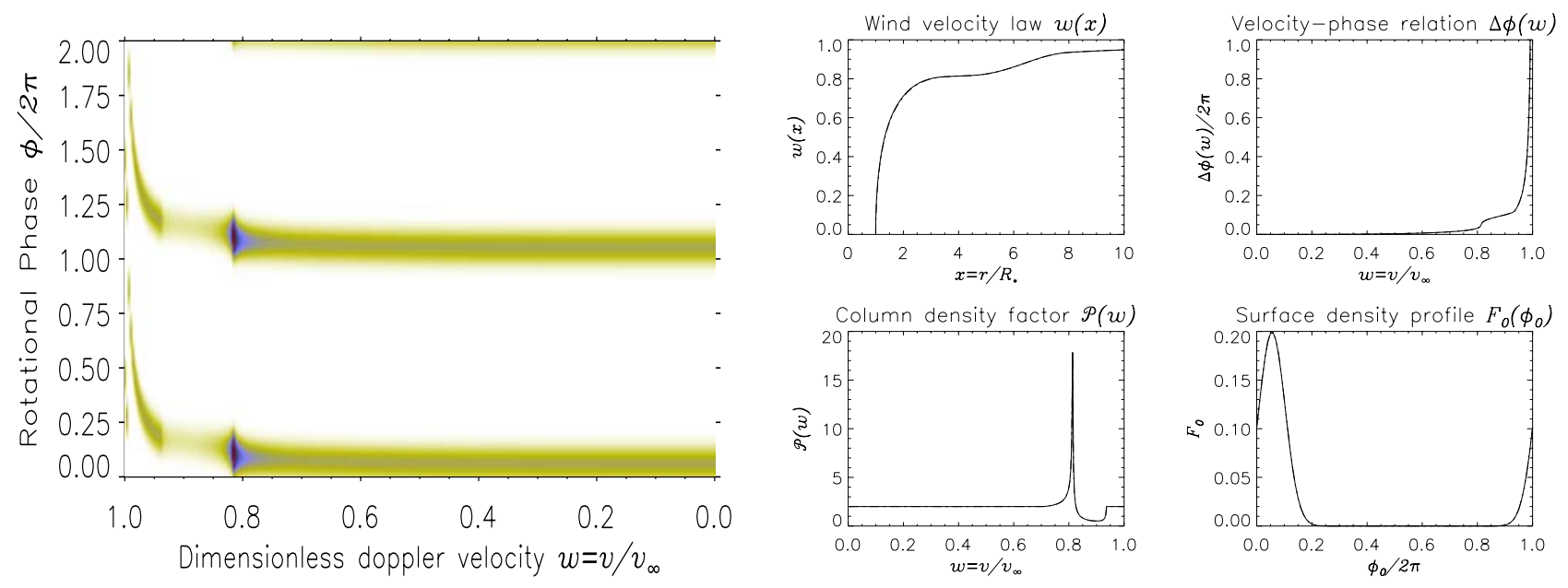

(c) Narrow plateau
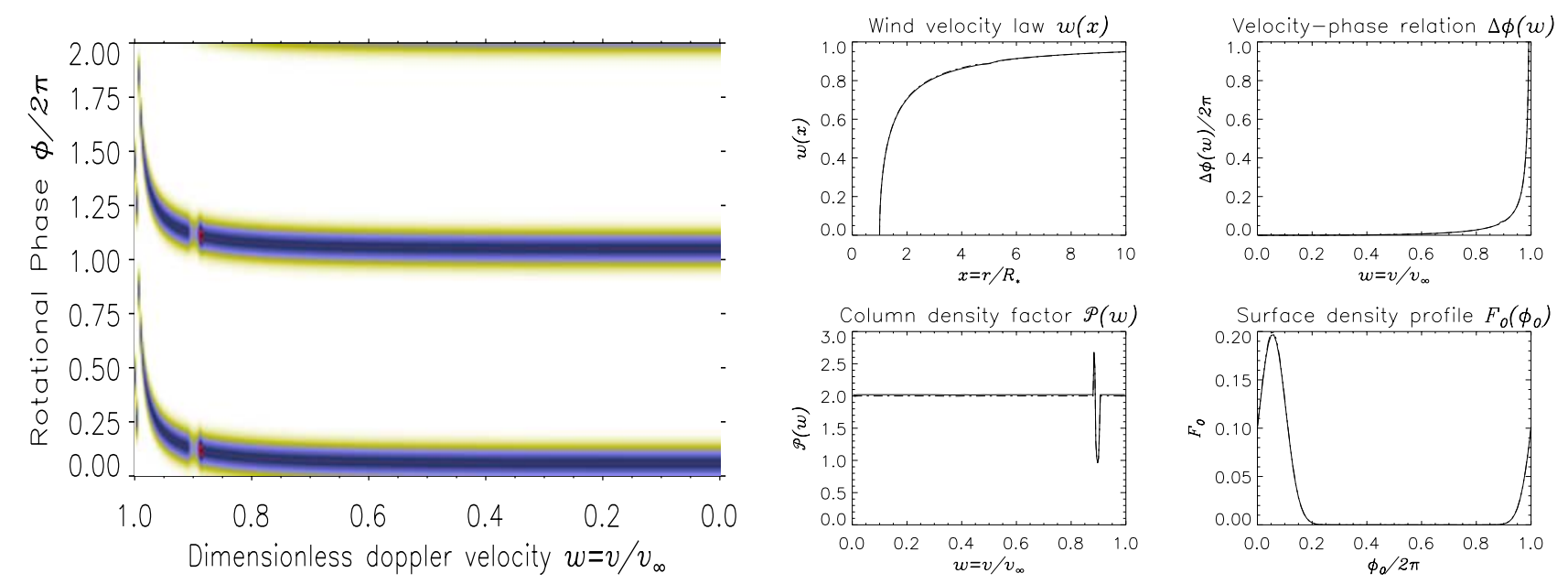

Fig. 7. $\left[\beta=\frac{1}{2}\right.$, noise free] As in Fig. 6, but for an underlying $\beta=\frac{1}{2}$ velocity law. 


\section{J. C. Brown et al.: Inference of density stream properties from DACs, Online Material p 4}

(a) Pure $\beta$-law
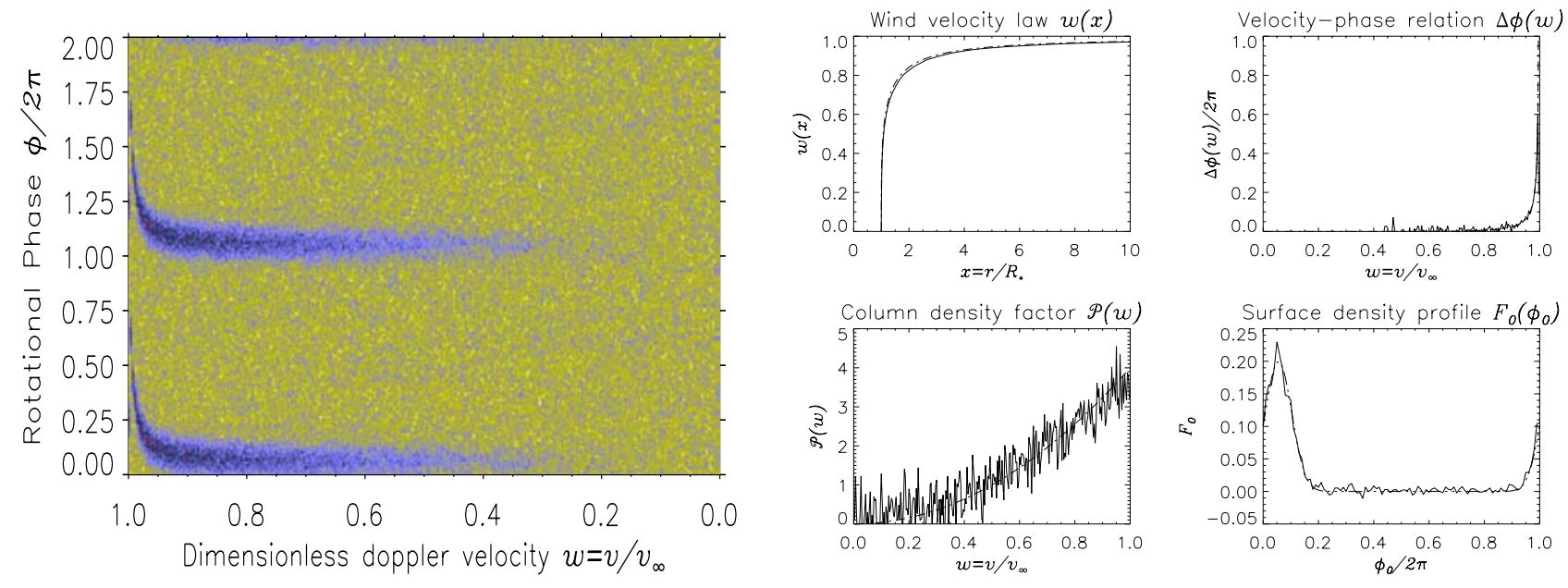

(b) Wide plateau
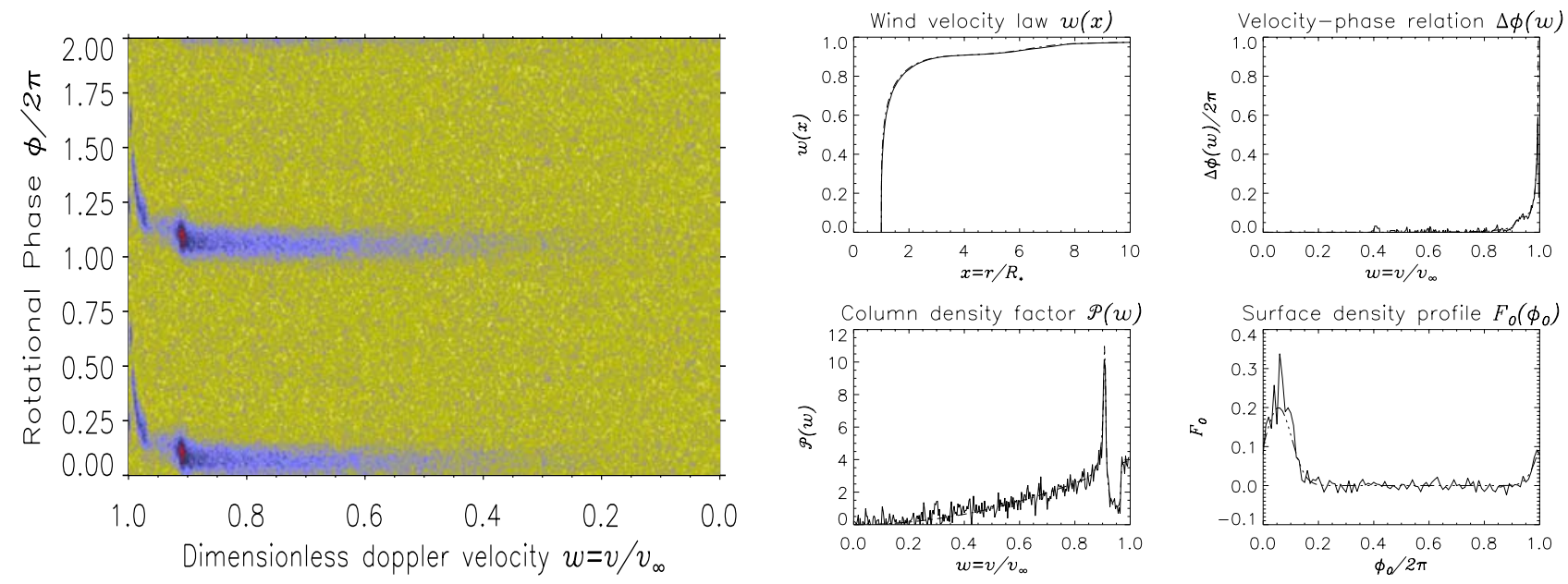

(c) Narrow plateau
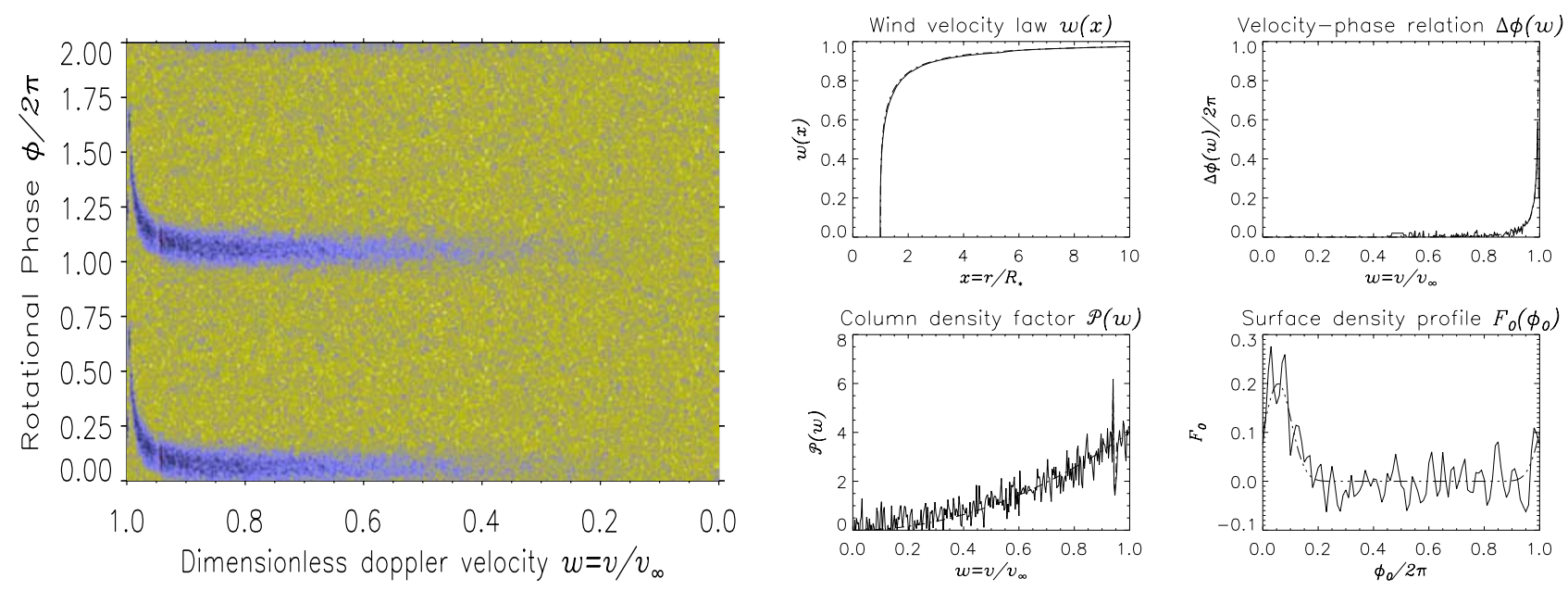

Fig. 9. $\left[\beta=\frac{1}{4}, 10 \%\right.$ errors] As in Fig. 6, but with $10 \%$ errors added to the dynamical spectrum. 
J. C. Brown et al.: Inference of density stream properties from DACs, Online Material p 5

(a) Pure $\beta$-law
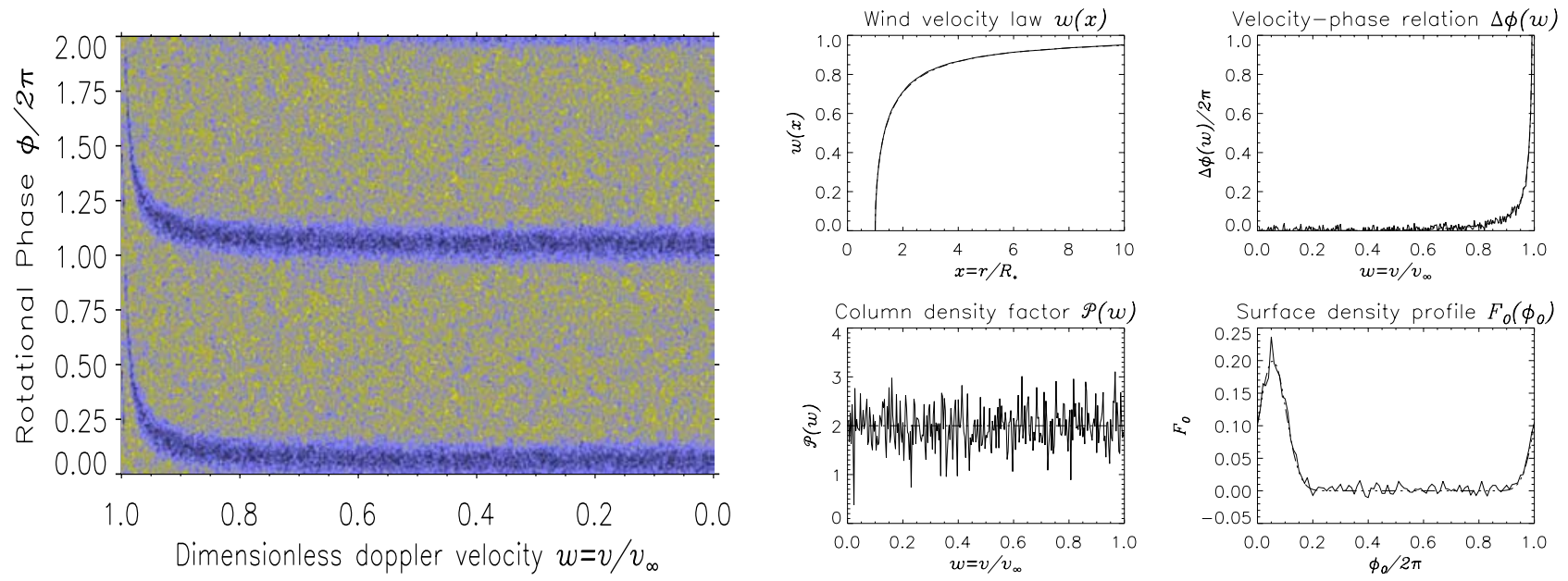

(b) Wide plateau
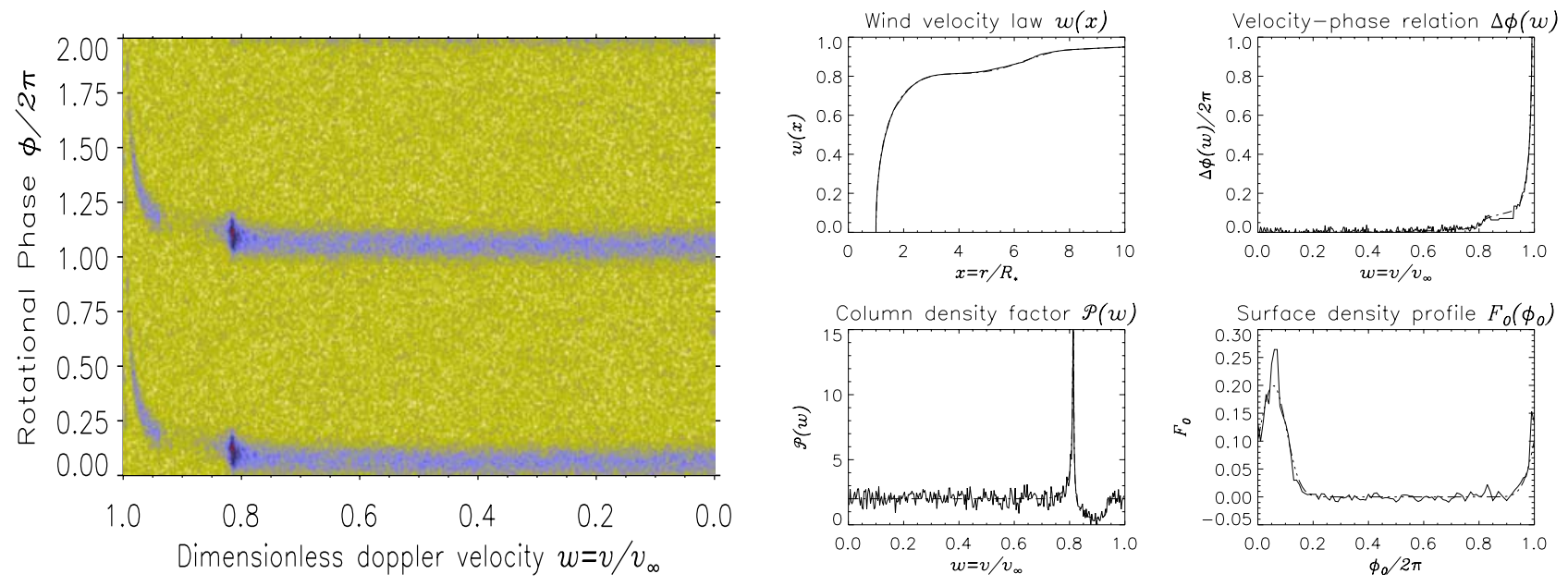

(c) Narrow plateau
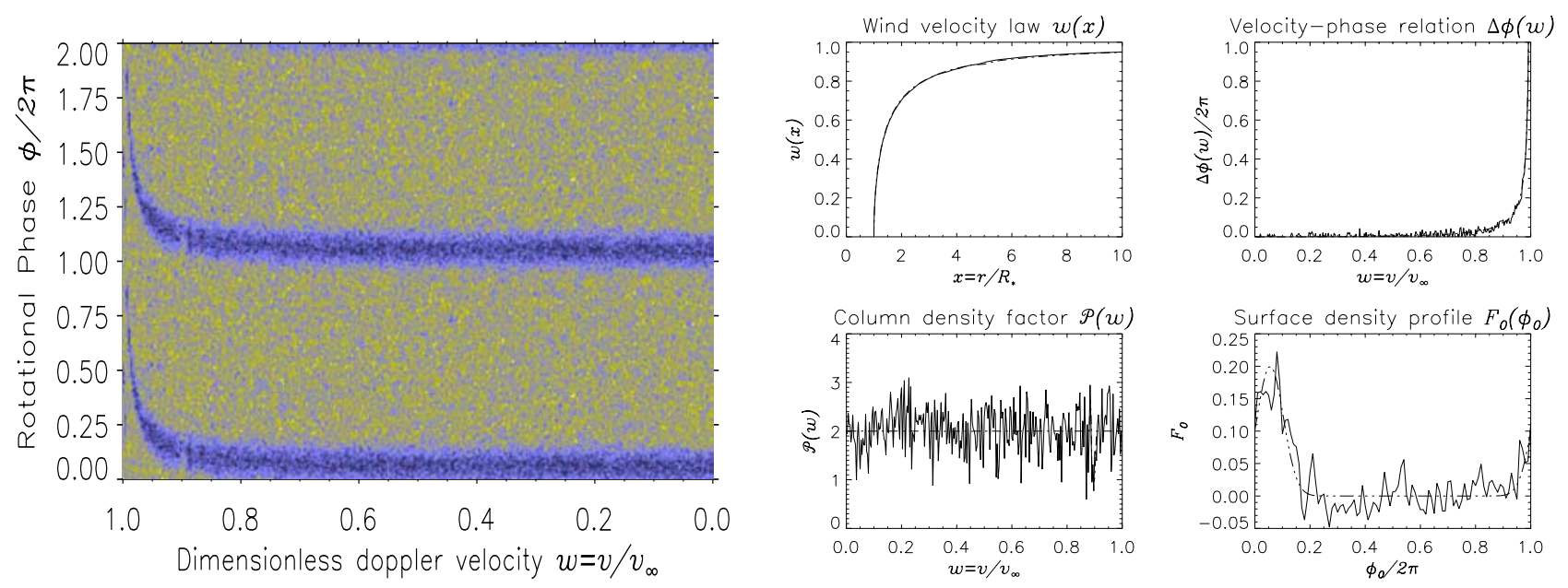

Fig. 10. $\left[\beta=\frac{1}{2}, 10 \%\right.$ errors $]$ As in Fig. 6, but for an underlying $\beta=\frac{1}{2}$ velocity law and $10 \%$ errors. 
J. C. Brown et al.: Inference of density stream properties from DACs, Online Material $p 6$

(a) Pure $\beta$-law
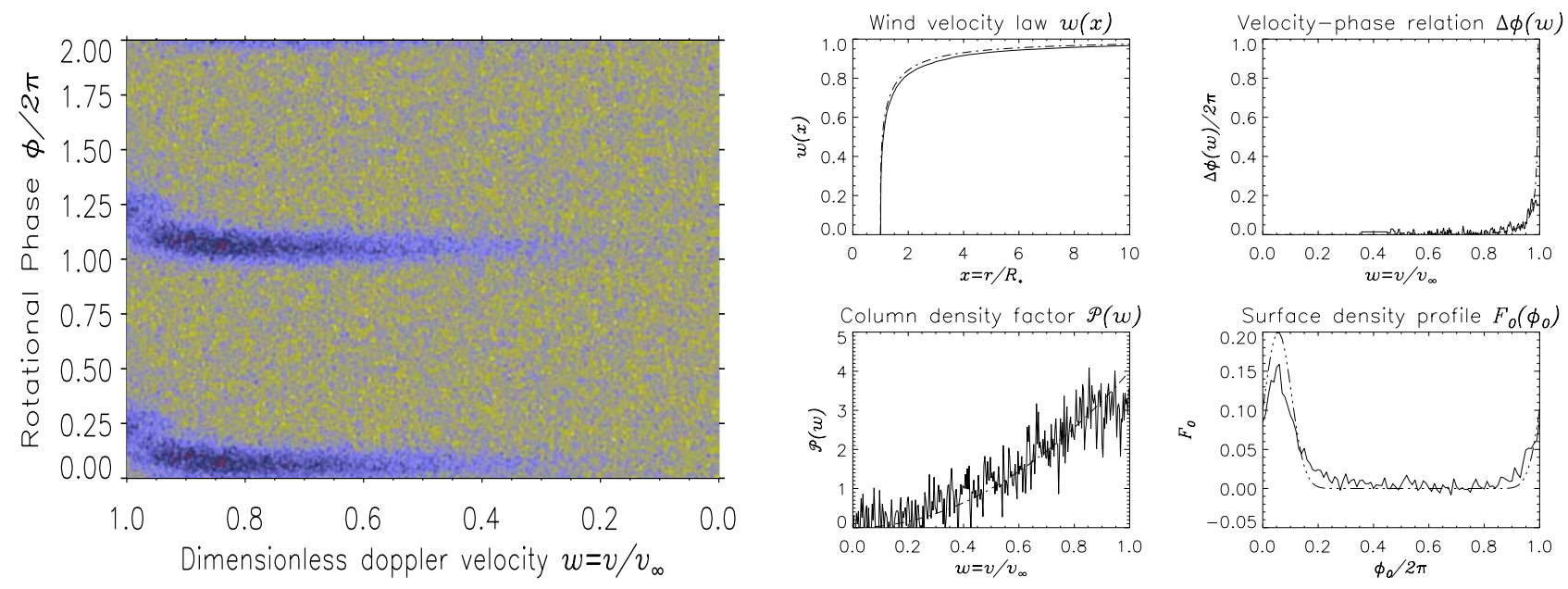

(b) Wide plateau
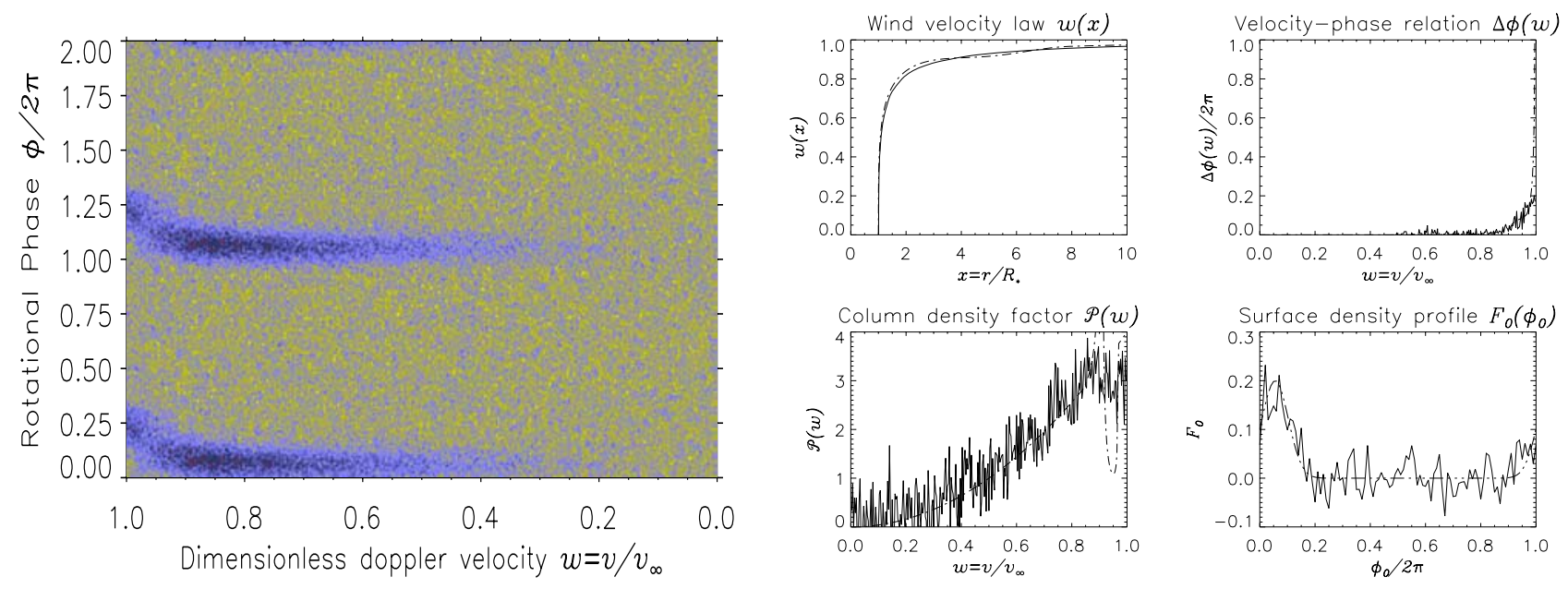

(c) Narrow plateau
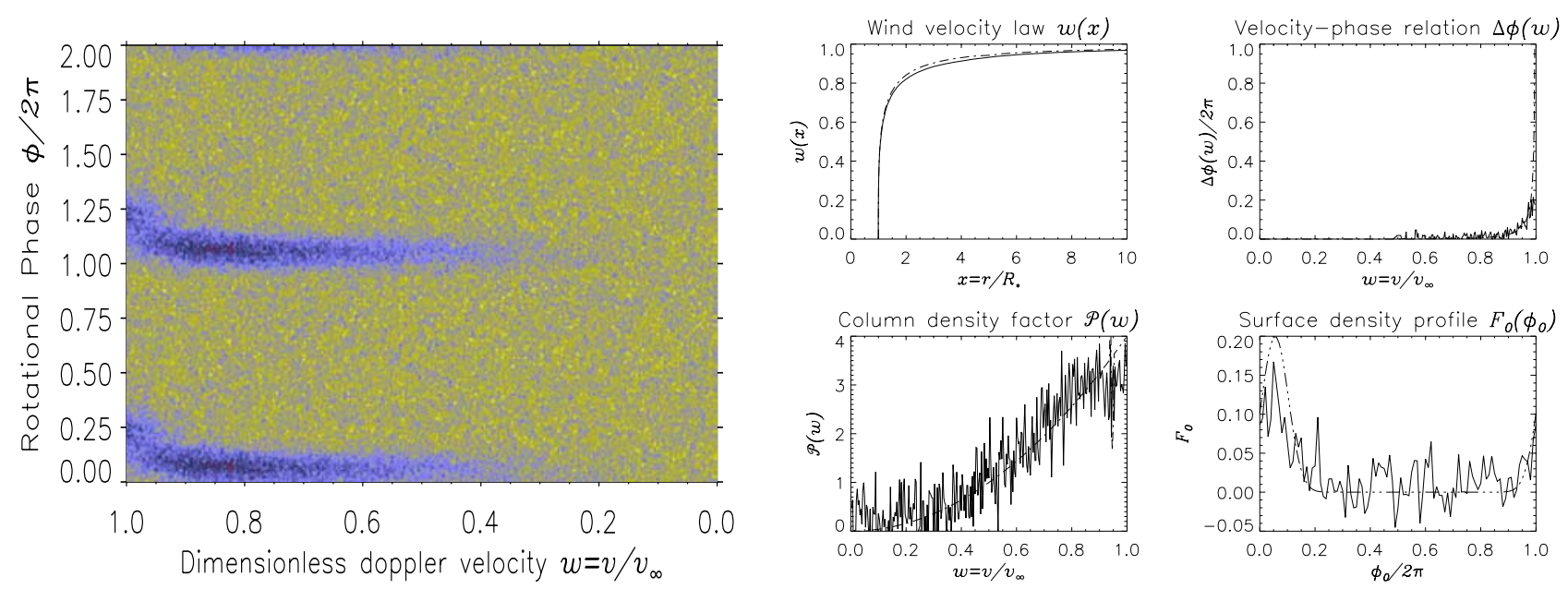

Fig. 12. $\left[\beta=\frac{1}{4}, 10 \%\right.$ errors, $5 \%$ smearing] As in Fig. 6, but with $10 \%$ errors added to the dynamical spectrum and "velocity blurring" at $5 \%$ of the terminal velocity. 
J. C. Brown et al.: Inference of density stream properties from DACs, Online Material $p 7$

(a) Pure $\beta$-law
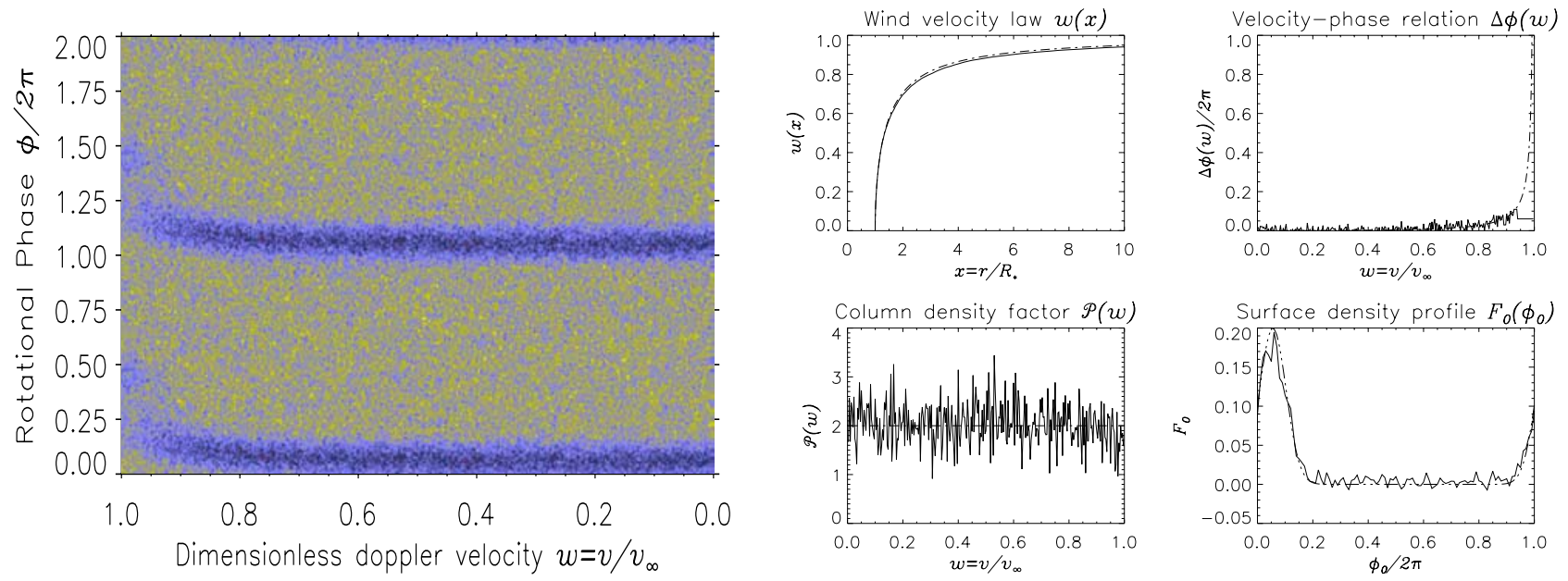

(b) Wide plateau
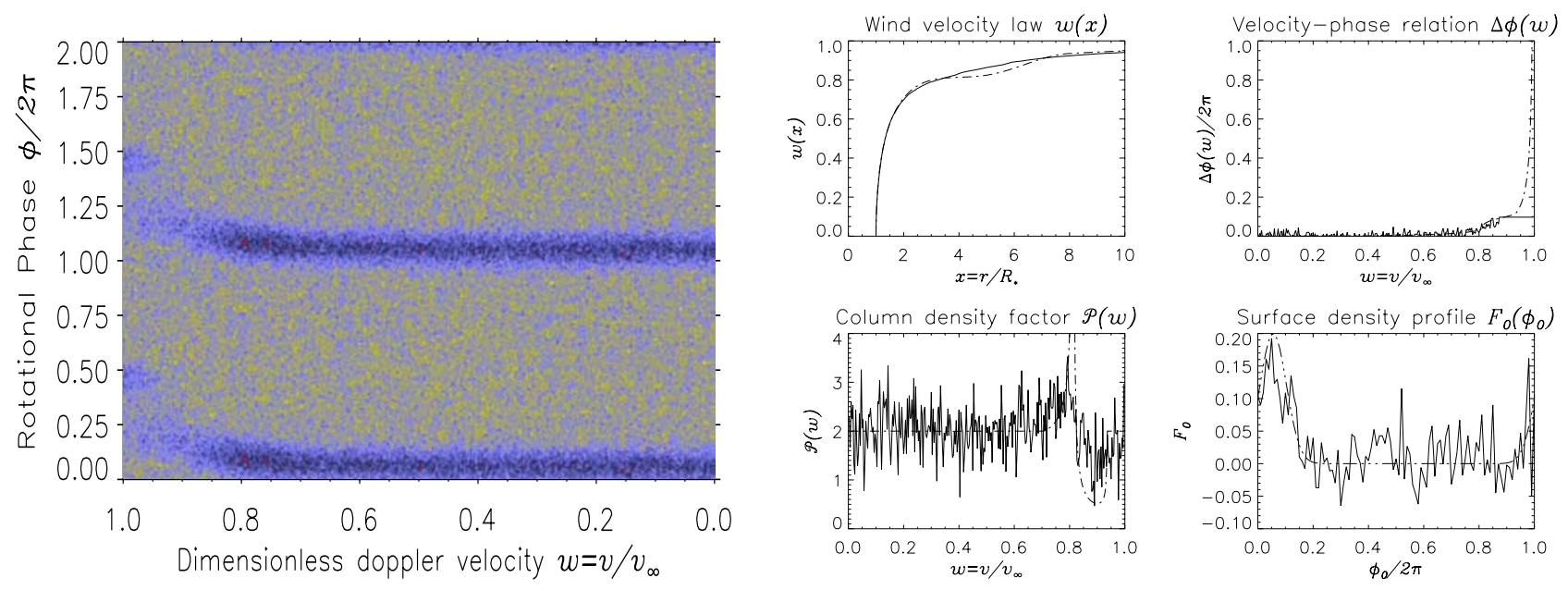

(c) Narrow plateau
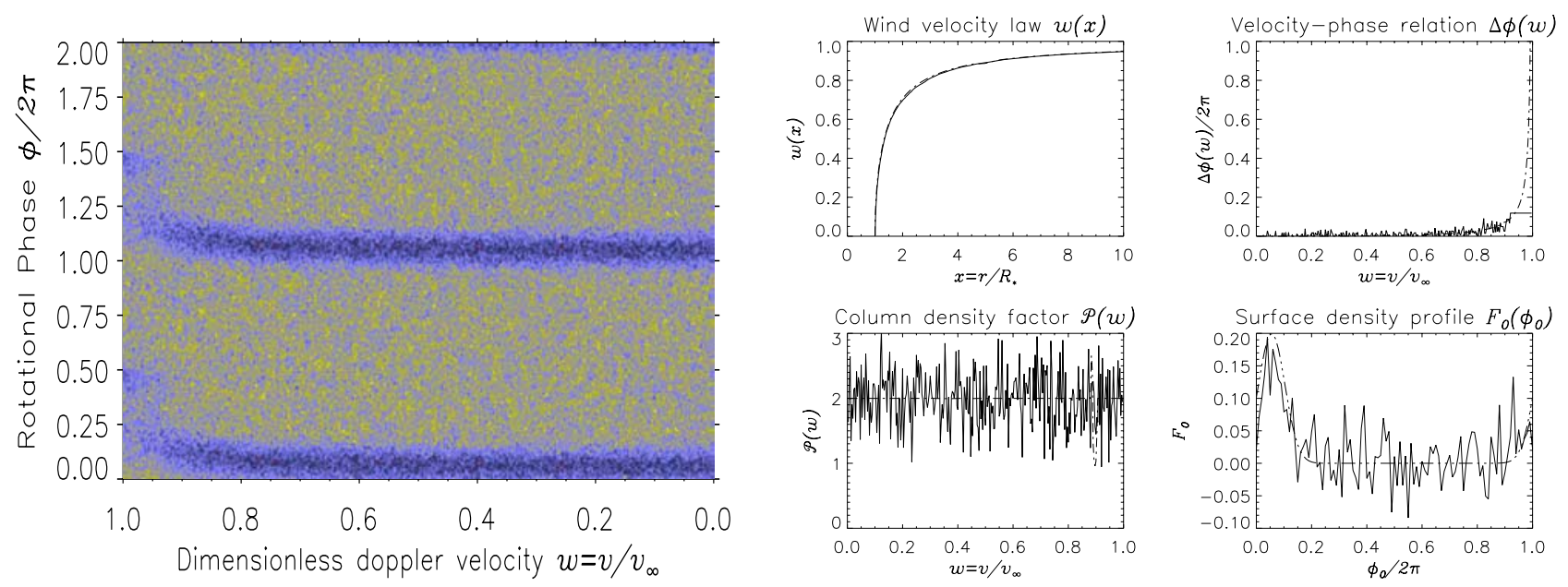

Fig. 13. $\left[\beta=\frac{1}{2}, 10 \%\right.$ errors, $5 \%$ smearing] As in Fig. 6, but for an underlying $\beta=\frac{1}{2}$ velocity law, $10 \%$ errors and "velocity blurring" at $5 \%$ of the terminal velocity. 
J. C. Brown et al.: Inference of density stream properties from DACs, Online Material $p 8$

(a) Pure $\beta$-law

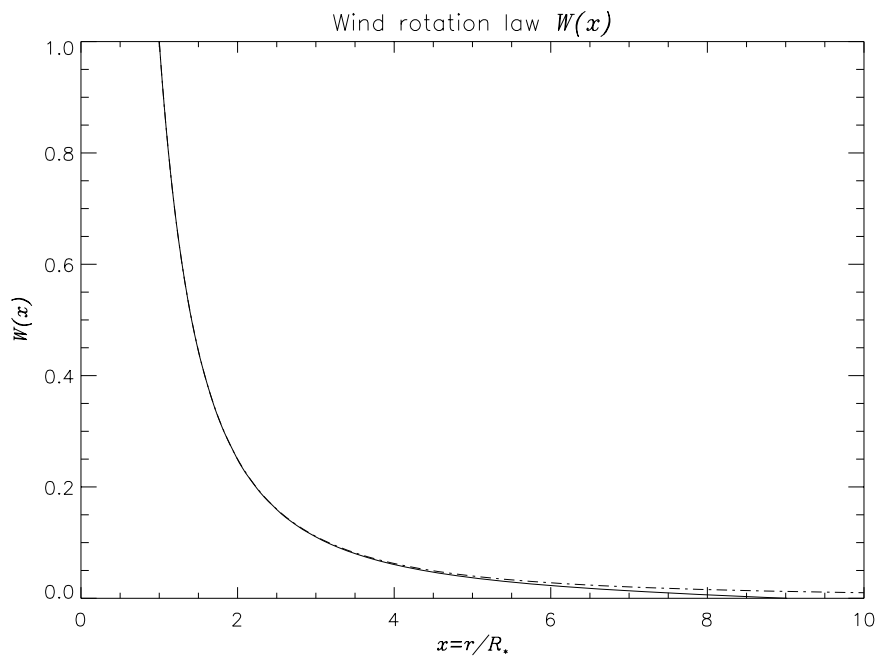

(b) Wide plateau

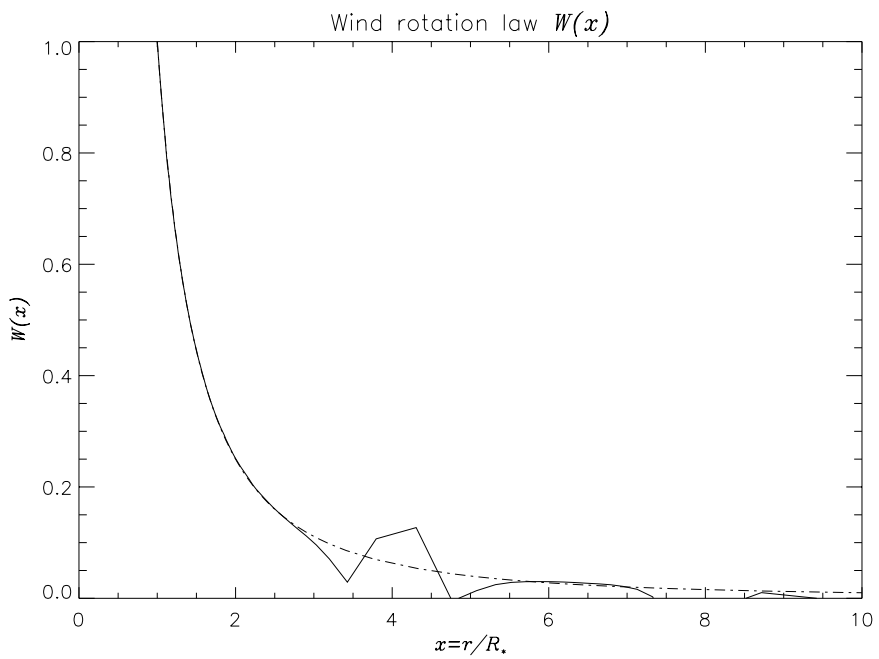

(c) Narrow plateau

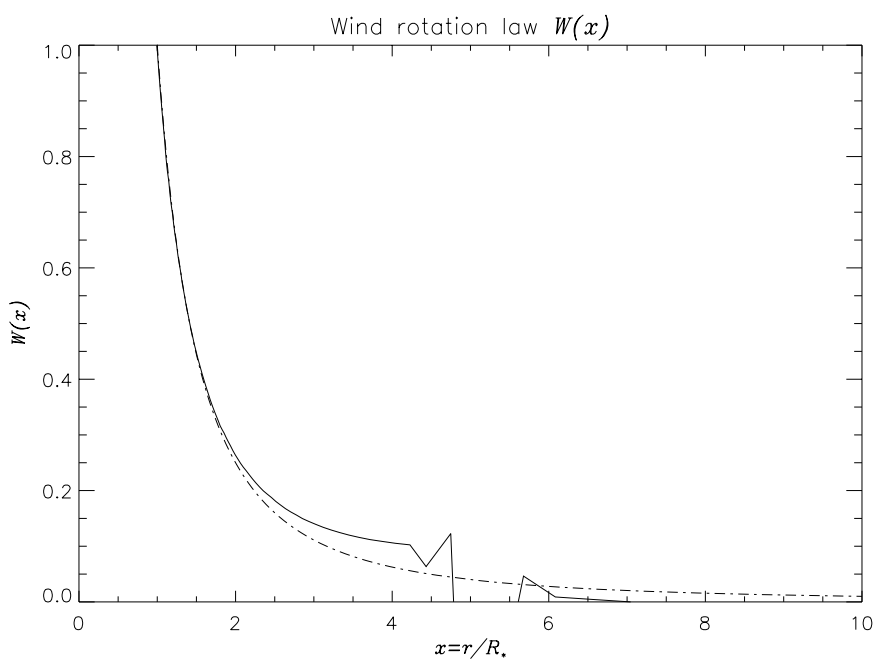

Fig. 15. $\left[\beta=\frac{1}{4}\right]$ Inferred wind rotation law, $W(x)$ (i.e., $\Omega(r)$ ) for the error-free dynamical spectra shown in the left panels of Fig. 6 , that is, for pure $\beta$-law, wide-plateau and narrow-plateau velocity laws, with an underlying $\beta=\frac{1}{4}$ velocity law. The true rotation law (constant angular momentum, $\left.W(x)=1 / x^{2}\right)$ is shown as a dashed line). 
J. C. Brown et al.: Inference of density stream properties from DACs, Online Material $p 9$

(a) Pure $\beta$-law

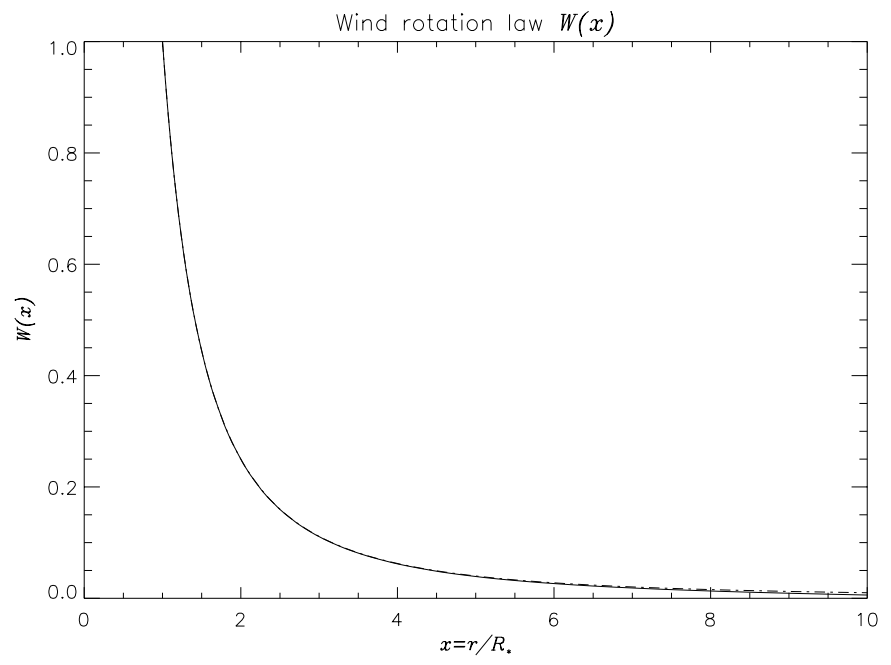

(b) Wide plateau

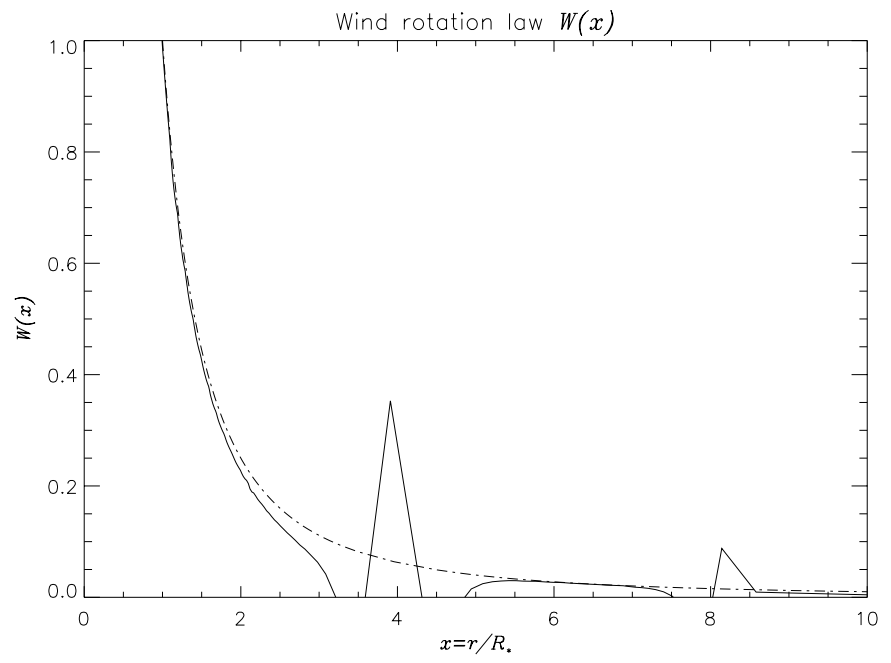

(c) Narrow plateau

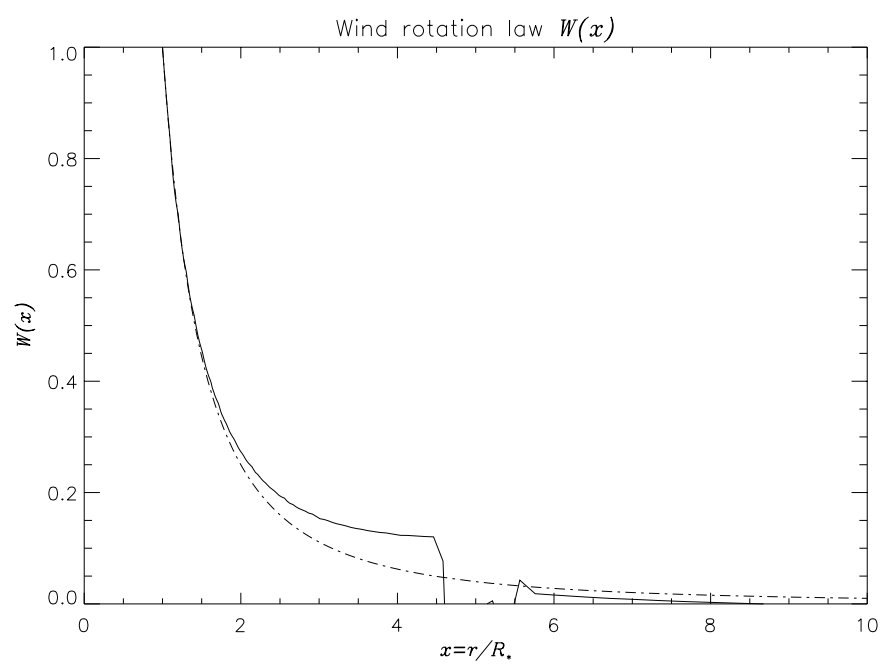

Fig. 16. $\left[\beta=\frac{1}{2}\right]$ As in Fig. 15, but for an underlying $\beta=\frac{1}{2}$ velocity law. 